June 24, 2018

\title{
Integrable highest weight modules over affine superalgebras and Appell's function
}

\author{
Victor G. Kac ${ }^{* \dagger}$ and Minoru Wakimoto ${ }^{\ddagger}$
}

\begin{abstract}
We classify integrable irreducible highest weight representations of non-twisted affine Lie superalgebras. We give a free field construction in the level 1 case. The analysis of this construction shows, in particular, that in the simplest case of the $s \ell(2 \mid 1)$ level 1 affine superalgebra the characters are expressed in terms of the Appell elliptic function. Our results demonstrate that the representation theory of affine Lie superalgebras is quite different from that of affine Lie algebras.
\end{abstract}

\section{Introduction}

In this paper we continue the study of integrable irreducible highest weight modules over affine superalgebras that we began in [KW].

First, let us recall the definition of an integrable module over an ordinary affine Kac-Moody algebra $\widehat{\mathfrak{g}}[\mathrm{K} 3]$. Let $\mathfrak{g}$ be a finite-dimensional simple or abelian Lie algebra over $\mathbb{C}$ with a symmetric invariant bilinear form (.|.). Recall that the associated affine algebra is

$$
\widehat{\mathfrak{g}}=\left(\mathbb{C}\left[t, t^{-1}\right] \otimes_{\mathbb{C}} \mathfrak{g}\right) \oplus \mathbb{C} K \oplus \mathbb{C} d
$$

with the following commutation relations $\left(a, b \in \mathfrak{g} ; m, n \in \mathbb{Z}\right.$ and $a(m)$ stands for $\left.t^{m} \otimes a\right)$ :

$$
[a(m), b(n)]=[a, b](m+n)+m \delta_{m,-n}(a \mid b) K, \quad[d, a(m)]=-m a(m), \quad[K, \widehat{\mathfrak{g}}]=0 .
$$

We identify $\mathfrak{g}$ with the subalgebra $1 \otimes \mathfrak{g}$. The bilinear form (.|.) extends from $\mathfrak{g}$ to a symmetric invariant bilinear form on $\widehat{\mathfrak{g}}$ by:

$$
\begin{aligned}
(a(m) \mid b(n)) & =\delta_{m,-n}(a \mid b),\left(\mathbb{C}\left[t, t^{-1}\right] \otimes \mathfrak{g} \mid \mathbb{C} K+\mathbb{C} d\right)=0 \\
(K \mid K) & =(d \mid d)=0, \quad(K \mid d)=-1
\end{aligned}
$$

\footnotetext{
${ }^{*}$ Department of Mathematics, M.I.T., Cambridge, MA 02139, USA. kac@math.mit.edu

${ }^{\dagger}$ Supported in part by NSF grant DMS-9970007.

${ }^{\ddagger}$ Graduate School of Mathematics, Kyushu University, Fukuoka 812-81, Japan wakimoto@math.kyushuu.ac.jp
} 
Choose a Cartan subalgebra $\mathfrak{h}$ of $\mathfrak{g}$ and let $\mathfrak{g}=\mathfrak{h} \oplus\left(\oplus_{\alpha \in \Delta} \mathfrak{g}_{\alpha}\right)$ be the root space decomposition, where $\mathfrak{g}_{\alpha}$ denotes the root space attached to a root $\alpha \in \Delta \subset \mathfrak{h}^{*}$. Let

$$
\widehat{\mathfrak{h}}=\mathfrak{h}+\mathbb{C} K+\mathbb{C} d
$$

be the Cartan subalgebra of $\widehat{\mathfrak{g}}$, and, as before, let $\mathfrak{g}_{\alpha}(m)=t^{m} \otimes \mathfrak{g}_{\alpha}$.

A $\widehat{\mathfrak{g}}$-module $V$ is called integrable if the following two properties hold [K3]:

$$
\begin{aligned}
& \widehat{\mathfrak{h}} \text { is diagonalizable on } V, \\
& \text { all } \mathfrak{g}_{\alpha}(m)(\alpha \text { a root of } \mathfrak{g}, m \in \mathbb{Z}) \text { are locally finite on } V \text {. }
\end{aligned}
$$

(Property (0.6) means that $\operatorname{dim} U\left(\mathfrak{g}_{\alpha}(m)\right) v<\infty$ for any $v \in V$.)

It is easy to show that these two properties imply

$$
\mathfrak{g} \text { is locally finite on } V \text { (i.e., } \operatorname{dim} U(\mathfrak{g}) v<\infty \text { for any } v \in V \text { ). }
$$

Here and further $U(\mathfrak{a})$ denotes the universal enveloping algebra of a Lie (super)algebra $\mathfrak{a}$. Note also that condition (0.6) is vacuous if $\mathfrak{g}$ is abelian.

Let now $\mathfrak{g}=\mathfrak{g}_{0}+\mathfrak{g}_{\overline{1}}$ be a finite-dimensional Lie superalgebra over $\mathbb{C}$ with an even symmetric invariant bilinear for (.|.) (for a background on Lie superalgebras see [K1]). Recall that "even" means that $\left(\mathfrak{g}_{\overline{0}} \mid \mathfrak{g}_{\overline{1}}\right)=0$, "symmetric" means that (.|.) is symmetric on $\mathfrak{g}_{\overline{0}}$ and skewsymmetric on $\mathfrak{g}_{\overline{1}}$, and "invariant" means that $([a, b] \mid c)=(a \mid[b, c]), a, b, c \in \mathfrak{g}$. We shall assume, in addition, that $\mathfrak{g}_{\overline{0}}$ is reductive:

$$
\mathfrak{g}_{\overline{0}}=\oplus_{j=0}^{N} \mathfrak{g}_{\overline{0} j},
$$

where $\mathfrak{g}_{\overline{0} 0}$ is abelian and $\mathfrak{g}_{\overline{0} j}$ with $j \geq 1$ are simple Lie algebras.

The affine superalgebra $\widehat{\mathfrak{g}}$ associated to the Lie superalgebra $\mathfrak{g}$ and the bilinear form (.|.) is defined in exactly the same way as in the Lie algebra case by relations (0.2). Likewise, the invariant even symmetric bilinear form (.|.) on $\widehat{\mathfrak{g}}$ is defined by (0.3), and the Cartan subalgebra $\widehat{\mathfrak{h}}$ is defined by (0.4) after a choice of a Cartan subalgebra $\mathfrak{h}$ of $\mathfrak{g}_{\overline{0}}$. Note that for each $j \in\{0,1, \ldots, N\}$, the superalgebra $\widehat{\mathfrak{g}}$ contains an affine Kac-Moody algebra $\widehat{\mathfrak{g}}_{\overline{0}} j$ associated to $\mathfrak{g}_{\overline{0} j}$.

We shall see that condition (0.6) of integrability is too strong in the superalgebra case, as for most of the affine superalgebras it allows only trivial highest weight modules. This forces us to consider weaker conditions (cf. [KW]):

Definition 0.1. Given a subset $J \subset\{1, \ldots, N\}$, a $\widehat{\mathfrak{g}}$-module $V$ is called $J$-integrable if it satisfies conditions (0.5) and (0.X) and if it is integrable as $\widehat{\mathfrak{g}}_{\overline{0}}$-module for all $j \in J$.

Let $\mathfrak{g}=\mathfrak{h} \oplus\left(\oplus_{\alpha \in \Delta} \mathfrak{g}_{\alpha}\right)$ be a root space decomposition of the Lie superalgebra $\mathfrak{g}$ with respect to a Cartan subalgebra $\mathfrak{h}$ of $\mathfrak{g}_{\overline{0}}$. Choose a set of positive toots $\Delta_{+}$in $\Delta$ and let $\mathfrak{n}_{+}=\oplus_{\alpha \in \Delta_{+}} \mathfrak{g}_{\alpha}$. For each $\Lambda \in \widehat{\mathfrak{h}}^{*}$ one defines an irreducible highest weight module $L(\Lambda)$ over $\widehat{\mathfrak{g}}$ as the (unique) irreducible $\widehat{\mathfrak{g}}$-module for which there exists a non-zero vector $v_{\Lambda}$ such that

$$
h v_{\Lambda}=\Lambda(h) v_{\Lambda} \text { for } h \in \widehat{\mathfrak{h}}, \mathfrak{n}_{+} v_{\Lambda}=0, \mathfrak{g}(m) v_{\Lambda}=0 \text { for } m>0,
$$


where, as before $\mathfrak{g}(m)=t^{m} \otimes \mathfrak{g}$. The number $k=\Lambda(K)$ is called the level of $L(\Lambda)$ and of $\Lambda$. Note that $K=k I$ on $L(\Lambda)$ and that $\bar{L}(\Lambda):=U(\mathfrak{g}) v_{\Lambda}$ is an irreducible highest weight module over $\mathfrak{g}$.

In $\S 1$ we describe a general approach to the classification of irreducible integrable highest weight modules over arbitrary Kac-Moody superalgebras, and in $\S$ 2 and $\S 6$ give their complete classification in the affine (non-twisted) case, using Serganova's odd reflections.

In $\S 3$ we give a free field realization of all level 1 integrable highest weight modules over $g \ell(m \mid n)$, which leads to a "quasiparticle" character formula for these modules and to a "theta function" type character formula. This construction may be viewed as a generalization of the classical boson-fermion correspondence based on the oscillator algebra $g \ell(1)$ and of the super boson-fermion correspondence based on $g \ell(1 \mid 1)^{\Upsilon} \mathrm{KL}$. The former produces the classical vertex operators and relates representation theory of $g \ell(1)$ to the denominator identity for $s \ell(2)$, while the latter produces vertex operators for the symplectic bosons and relates representation theory of $g \ell(1 \mid 1)$ to the denominator identity for $s \ell(2 \mid 1)$ (see [K4]).

In $\S$ we show that the "theta function" type character formula for $s \ell(m \mid 1)(m \geq 2)$ is a product of a theta function, a power of the eta function, and a more "exotic" function, called a multivariable Appell function. The classical Appell function appeared in the 1880's in the papers by Appell [A] and by Hermite in their study of elliptic functions. Most recently this function has been discussed in $[\mathbb{P}]$. The study of asymptotics of Appell's functions gives the high temperature asymptotics of integrable level $1 \mathrm{sl}(m \mid 1)$-characters. We also derive here formulas for branching functions for integrable level $1 s \ell(m \mid 1)$-modules restricted to the even subalgebra. They turn out to be certain "half" modular functions.

In $\S$ f we relate integrable level 1 modules over $g \ell(m \mid n)$ to the denominator identity for $s \ell(m+1 \mid n)$, and as a result, we derive for these modules yet another, a Weyl type, character formula.

In $\S$ we give a free field realization of the two level 1 integrable highest weight modules over $\operatorname{ssp}(m \mid n)^{\tilde{r}}$, which generalizes the constructions for $s o(m)^{\Upsilon}$ and $s p(n)^{\Upsilon}$ from [KP1], [F] and [FF]. These lead to character formulas and high temperature asymptotics of the characters.

In $\S 8$ we show that integrability is a necessary condition for an irreducible highest weight $\widehat{\mathfrak{g}}$-module to be a module over the associated vertex algebra, and that in the level 1 case this condition is sufficient. We thus get examples of rational vertex algebras for which the $\mathbb{C}$-span of normalized (super)characters is not $S L(2, \mathbb{Z})$-invariant. The latter property was proved in [Z] under certain additional assumptions, and it was generally believed that these assumptions were superfluous.

In $\S 9$ we discuss some open problems.

It is interesting to note that in the "super" case a number of new interesting phenomena occur. The level gets quantized by the integrability condition, but in almost all cases the number of integrable modules is infinite. This is the case for the lowest, level 1, integrable $s \ell(m \mid n)$-modules which apparently causes the specialized characters and branching functions to lose their customary modularity properties, which are so ubiquitous in the affine Lie algebra case [KP2], [K3]. However, in the cases when the number of characters of given level is finite, like, for example, $k=1 \quad o s p(m \mid n)$ case, the specialized normalized characters are still modular, though their $\mathbb{C}$-span is no longer $S L(2, \mathbb{Z})$-invariant as in the affine Lie algebra case.

It is also interesting to note that while the characters of affine Lie algebras are global sections of line bundles on abelian varieties, the characters of affine Lie superalgebras are related to global 
sections of rank 2 vector bundles on abelian varieties, as the work of Polishchuk [P] on Appell's function apparently indicates.

We are grateful to A. Polishchuk for giving us the idea of Lemma 4.1 and other very useful remarks. The first named author wishes to thank ENS, IHES and MSRI for their hospitality.

\section{Integrability of highest weight modules over Kac-Moody superalgebras}

Consider the following data:

$$
\mathcal{D}=\left\{\mathfrak{h}, I, I_{1}, \Pi^{\vee}, \Pi\right\}
$$

where $\mathfrak{h}$ is a vector space, $I$ is an index set, $I_{1}$ is a subset of $I, \Pi^{\vee}=\left\{\alpha_{i}^{\vee}\right\}_{i \in I}$ and $\Pi=\left\{\alpha_{i}\right\}_{i \in I}$ are linearly independent sets of vectors in $\mathfrak{h}$ and $\mathfrak{h}^{*}$ respectively indexed by $I$. One associates to these data a Lie superalgebra $\mathfrak{g}(\mathcal{D})$ defined as the quotient of the Lie algebra on generators $e_{i}, f_{i}(i \in I)$ and $\mathfrak{h}$, the generators $e_{i}$ and $f_{i}$ for $i \in I_{1}$ being odd and all other generators being even, and the standard relations $(i, j \in I, h \in \mathfrak{h})$ :

$$
[\mathfrak{h}, \mathfrak{h}]=0, \quad\left[e_{i}, f_{j}\right]=\delta_{i j} \alpha_{i}^{\vee}, \quad\left[h, e_{i}\right]=\left\langle\alpha_{i}, h\right\rangle e_{i}, \quad\left[h, f_{i}\right]=-\left\langle\alpha_{i}, h\right\rangle f_{i},
$$

by the maximal graded with respect to the root space decomposition intersecting $\mathfrak{h}$ trivially (cf. [K1], K3] ).

The commutative ad-diagonizable subalgebra $\mathfrak{h}$ of $\mathfrak{g}(\mathcal{D})$ is called the Cartan subalgebra, $\Pi$ and $\Pi^{\vee}$ are called the sets of simple roots and coroots respectively, elements $e_{i}$ and $f_{i}(i \in I)$ are called Chevalley generators, etc. One defines the notions of roots and root spaces in the usual way (cf. [K1], K3]). Let $\mathfrak{n}_{+}\left(\right.$resp. $\left.\mathfrak{n}_{-}\right)$denote the subalgebra of $\mathfrak{g}$ generated by the $e_{i}$ 's (resp. $f_{i}$ 's). Then, as usual, one has the triangular decomposition:

$$
\mathfrak{g}=\mathfrak{n}_{-}+\mathfrak{h}+\mathfrak{n}_{+}
$$

Let $a_{i j}=\left\langle\alpha_{j}, \alpha_{i}^{\vee}\right\rangle$. The matrix $A=\left(a_{i j}\right)_{i, j \in I}$ is called the Cartan matrix of the data $\mathcal{D}$ (and of $\mathfrak{g}(\mathcal{D}))$.

A root of $\mathfrak{g}(\mathcal{D})$ is called even (resp. odd) if the attached root space is even (resp. odd). For example a simple root $\alpha_{s}$ is called odd iff $s \in I_{1}$. An odd simple root $\alpha_{s}$ (and the coroot $\alpha_{s}^{\vee}$ ) is called isotropic if $a_{s s}=0$. In what follows we let

$$
p_{i j}=\left\{\begin{aligned}
-1 & \text { if both } \alpha_{i} \text { and } \alpha_{j} \text { are odd } \\
1 & \text { othewise }
\end{aligned}\right.
$$

Note that $\mathfrak{g}(\mathcal{D})$ has an anti-involution $\omega$ defined by $\omega\left(e_{i}\right)=f_{i}, \omega\left(f_{i}\right)=e_{i},\left.\omega\right|_{\mathfrak{h}}=I$. For that reason properties of the $e_{i}$ 's automatically hold for the $f_{i}$ 's.

Lemma 1.1. (a) An odd simple root $\alpha_{i}$ is isotropic iff $\left[e_{i}, e_{i}\right]=0$.

(b) If $i \neq j$, then $\left[e_{i}, e_{j}\right]=0$ iff $a_{i j}=a_{j i}=0$.

Proof. It is clear that $\left[f_{j},\left[e_{i}, e_{i}\right]\right]=0$ if $j \neq i$, and one has: $\left[f_{i},\left[e_{i}, e_{i}\right]\right]=2 a_{i i} e_{i}$, which proves (a). The proof of (b) is similar. 
It is straightforward to check the following relation $(i, j \in I, i \neq j)$ :

$$
\left[\left[e_{i}, e_{j}\right],\left[f_{i}, f_{j}\right]\right]=p_{i j}\left(a_{i j} \alpha_{j}^{\vee}-p_{i i} a_{j i} \alpha_{i}^{\vee}\right) .
$$

Further on we shall always assume the following property of the Cartan matrix $A$ :

$$
a_{i j}=0 \text { iff } a_{j i}=0 .
$$

Given $s \in I_{1}$ such that $a_{s s}=0$ (i.e., $\alpha_{s}$ is an odd isotropic simple root), define a new data

$$
r_{s}(\mathcal{D})=\left\{\mathfrak{h}, I, r_{s}\left(I_{1}\right), r_{s}\left(\Pi^{\vee}\right), r_{s}(\Pi)\right\}
$$

and new Chevalley generators $r_{s}\left(e_{i}\right), r_{s}\left(f_{i}\right)$ of $\mathfrak{g}(\mathcal{D})$ as follows (cf. [S], [PS], [ [KW]):

$$
\begin{aligned}
i \in r_{s}\left(I_{1}\right) \text { iff } i & \notin I_{1} \text { in case } a_{s i} \neq 0, \quad i \in r_{s}\left(I_{1}\right) \text { iff } i \in I_{1} \text { otherwise; } \\
r_{s}\left(\alpha_{s}^{\vee}\right) & =-\alpha_{s}^{\vee}, \quad r_{s}\left(\alpha_{s}\right)=-\alpha_{s}, \\
r_{s}\left(\alpha_{i}^{\vee}\right) & =\alpha_{i}^{\vee}+\frac{a_{i s}}{a_{s i}} \alpha_{s}^{\vee} \text { and } r_{s}\left(\alpha_{i}\right)=\alpha_{i}+\alpha_{s} \text { if } a_{s i} \neq 0, \\
r_{s}\left(\alpha_{i}^{\vee}\right) & =\alpha_{i}^{\vee} \text { and } r_{s}\left(\alpha_{i}\right)=\alpha_{i} \text { in all other cases; } \\
r_{s}\left(e_{s}\right) & =f_{s}, \quad r_{s}\left(f_{s}\right)=-e_{s}, \\
r_{s}\left(e_{i}\right) & =\left[e_{s}, e_{i}\right] \text { and } r_{s}\left(f_{i}\right)=\frac{1}{p_{s i} a_{s i}}\left[f_{s}, f_{i}\right] \text { if } a_{s i} \neq 0 \\
r_{s}\left(e_{i}\right) & =e_{i}, \quad r_{s}\left(f_{i}\right)=f_{i} \text { in all other cases. }
\end{aligned}
$$

Denote by $r_{s}\left(\mathfrak{n}_{+}\right)$(resp. $r_{s}\left(\mathfrak{n}_{-}\right)$) the subalgebra of $\mathfrak{g}(\mathcal{D})$ generated by the $r_{s}\left(e_{i}\right)$ 's $\left(\right.$ resp. $r_{s}\left(f_{i}\right)$ 's). The transformation $r_{s}$ is called an odd reflection (with respect to $\alpha_{s}$ ).

Lemma 1.2. (a) The data $r_{s}(\mathcal{D})$ satisfy (1.2).

(b) The new Chevalley generators satisfy the standard relations and together with $\mathfrak{h}$ generate $\mathfrak{g}(\mathcal{D})$, so that $\mathfrak{g}\left(r_{s}(\mathcal{D})\right) \simeq \mathfrak{g}(\mathcal{D})$.

(c) One has the new triangular decomposition:

$$
\mathfrak{g}(\mathcal{D})=r_{s}\left(\mathfrak{n}_{-}\right)+\mathfrak{h}+r_{s}\left(\mathfrak{n}_{+}\right) .
$$

(d) The data $r_{s}\left(r_{s}(\mathcal{D})\right)$ coincide with $\mathcal{D}$, and the Chevalley generators $r_{s}\left(r_{s}\left(e_{i}\right)\right)$ (resp. $\left.r_{s}\left(r_{s}\left(f_{i}\right)\right)\right)$ coincide, up to a non-zero factor, with $e_{i}$ (resp. $f_{i}$ ).

Proof. is straightforward using (1.1) and the relation

$$
[a,[a, b]]=\frac{1}{2}[[a, a], b] \text { if } a \text { ia an odd element. }
$$

An element $\rho \in \mathfrak{h}^{*}$ such that

$$
\left\langle\rho, \alpha_{i}^{\vee}\right\rangle=\frac{1}{2} a_{i i} \text { for all } i \in I
$$

is called a Weyl vector for $\Pi^{\vee}$. 
Lemma 1.3. If $\rho$ is a Weyl vector for $\Pi^{\vee}$, then $\rho+\alpha_{s}$ is a Weyl vector for $r_{s}\left(\Pi^{\vee}\right)$.

Proof. It suffices to check that in the case $a_{i s} \neq 0$ one has: $\left\langle\rho+\alpha_{s}, a_{s i} \alpha_{i}^{\vee}+a_{i s} \alpha_{s}^{\vee}\right\rangle=$ $\frac{1}{2}\left\langle\alpha_{i}+\alpha_{s}, a_{s i} \alpha_{i}^{\vee}+a_{i s} \alpha_{s}^{\vee}\right\rangle$, which is immediate.

Recall that for each $\Lambda \in \mathfrak{h}^{*}$ one defines an irreducible highest weight module $L(\Lambda)$ over $\mathfrak{g}(\mathcal{D})$ as the (unique) irreducible $\mathfrak{g}(\mathcal{D})$-module for which there exists a non-zero vector $v_{\Lambda}$ such that

$$
h v_{\Lambda}=\Lambda(h) v_{\Lambda} \text { for } h \in \mathfrak{h}, \quad \mathfrak{n}_{+} v_{\Lambda}=0 .
$$

The vector $v_{\Lambda}$, called a highest weight vector (with respect to $\mathfrak{n}_{+}$) is determined uniquely up to a (non-zero) constant factor by the condition $\mathfrak{n}_{+} v_{\Lambda}=0$ (cf. [K3]). The linear function $\Lambda$ is called the highest weight (with respect to $\mathfrak{n}_{+}$) of $L(\Lambda$ ).

Lemma 1.4. Let $\alpha_{s}$ be an odd isotropic simple root and let $\mathfrak{n}_{+}^{\prime}=r_{s}\left(\mathfrak{n}_{+}\right)$.

(a) If $\left\langle\Lambda, \alpha_{s}^{\vee}\right\rangle=0$, then $v_{\Lambda}^{\prime}=v_{\Lambda}$ is a highest weight vector with respect to $\mathfrak{n}_{+}^{\prime}$, so that the highest weight remains the same: $\Lambda^{\prime}=\Lambda$.

(b) If $\left\langle\Lambda, \alpha_{s}^{\vee}\right\rangle \neq 0$, then $v_{\Lambda}^{\prime}=f_{s} v_{\Lambda}$ is a highest weight vector with respect to $\mathfrak{n}_{+}^{\prime}$ so that the highest weight vector becomes $\Lambda^{\prime}=\Lambda-\alpha_{s}$.

Proof. is straightforward using the facts that $f_{s}^{2} v_{\Lambda}=\frac{1}{2}\left[f_{s}, f_{s}\right] v_{\Lambda}=0$, and $f_{s} v_{\Lambda}=0$ iff $\left\langle\Lambda, \alpha_{s}^{\vee}\right\rangle=0$.

As an immediate corollary of Lemmas 1.3 and 1.4 we obtain the following very useful formulas (cf. $\mathrm{KW})$ :

$$
\begin{aligned}
& \Lambda^{\prime}+\rho^{\prime}=\Lambda+\rho \text { if }\left\langle\Lambda, \alpha_{s}^{\vee}\right\rangle\left(=\left\langle\Lambda+\rho, \alpha_{s}^{\vee}\right\rangle\right) \neq 0, \\
& \Lambda^{\prime}+\rho^{\prime}=\Lambda+\rho+\alpha_{s} \text { if }\left\langle\Lambda, \alpha_{s}^{\vee}\right\rangle\left(=\left\langle\Lambda+\rho, \alpha_{s}^{\vee}\right\rangle\right)=0 .
\end{aligned}
$$

Let $\alpha \in \mathfrak{h}^{*}$ be a positive even root of $\mathfrak{g}(\mathcal{D})$ such that there exist root vectors $e$ attached to $\alpha$ and $f$ attached to $-\alpha$ satisfying the following conditions:

(i) ad $f$ is locally nilpotent on $\mathfrak{g}(\mathcal{D})$,

(ii) $[e, f]=\alpha^{\vee} \in \mathfrak{h}, \quad\left[\alpha^{\vee}, e\right]=2 e, \quad\left[\alpha^{\vee}, f\right]=-2 f$.

Then we call $f$ an integrable element of $\mathfrak{g}(\mathcal{D})$. The following lemma is well-known (cf. [K3]).

Lemma 1.5. Let $f$ be an integrable element attached to a negative root $\alpha$.

(a) If $f$ is locally nilpotent on $L(\Lambda)$ then $\left\langle\Lambda, \alpha^{\vee}\right\rangle \in \mathbb{Z}_{+}$.

(b) Provided that $\alpha$ is a simple root, $f$ is locally nilpotent on $L(\Lambda)$ iff $\left\langle\Lambda, \alpha^{\vee}\right\rangle \in \mathbb{Z}_{+}$. 
Let $\beta=\alpha_{s}$ be an odd isotropic simple root. It will be convenient to use notation $r_{\beta}$ in place of $r_{s}$. Consider a sequence of roots $\beta_{0}, \beta_{1}, \ldots, \beta_{k}$ such that $\beta_{0}$ is an odd isotropic simple root from $\Pi^{(0)}:=\Pi, \beta_{1}$ is an odd isotropic simple root from $\Pi^{(1)}=r_{\beta_{0}}\left(\Pi^{(0)}\right), \ldots, \beta_{k}$ is an odd isotropic simple root from $\Pi^{(k)}=r_{\beta_{k-1}}\left(\Pi^{(k-1)}\right)$. Given $\Lambda \in \mathfrak{h}^{*}$, let $\Lambda^{(0)}=\Lambda$ be the highest weight of $L(\Lambda)$ with respect to $\mathfrak{n}_{+}^{(0)}:=\mathfrak{n}_{+}, \Lambda^{(1)}$ be the highest weight of $L(\Lambda)$ with respect to $\mathfrak{n}_{+}^{(1)}:=r_{\beta_{0}}\left(\mathfrak{n}_{+}\right), \ldots, \Lambda^{(k)}$ be the highest weight of $L(\Lambda)$ with respect to $\mathfrak{n}_{+}^{(k)}=r_{\beta_{k-1}}\left(\mathfrak{n}_{+}^{(k-1)}\right)$. Let $\rho^{(k)}$ be a Weyl vector for $\Pi^{(k)}$.

Proposition 1.1. Let $\alpha$ be a positive root of $\mathfrak{g}(\mathcal{D})$ and let $f$ be an integrable root element attached to $-\alpha$. Given $\Lambda \in \mathfrak{h}^{*}$, let

$$
S=\left\{i \in[0,1, \ldots, k-1] \mid\left\langle\Lambda^{(i)}, \beta_{i}^{\vee}\right\rangle=0\right\} .
$$

Suppose that $\alpha \in \Pi^{(k)}$. Then the element $f$ is locally nilpotent on $L(\Lambda)$ if and only if

$$
\left\langle\Lambda+\rho+\sum_{i \in S} \beta_{i}, \alpha^{\vee}\right\rangle \in \mathbb{N}=\{1,2, \ldots\}
$$

Proof. It follows from (1.4) that

$$
\Lambda^{(k)}+\rho^{(k)}=\Lambda+\rho+\sum_{i \in S} \beta_{i}
$$

Since $\left\langle\Lambda^{(k)}+\rho^{(k)}, \alpha^{\vee}\right\rangle=\left\langle\Lambda^{(k)}, \alpha^{\vee}\right\rangle+1$, the proposition follows from Lemma 1.5b.

Proposition 1.2. If, under the assumptions of Proposition 1.1, one has:

$$
\left\langle\Lambda+\rho, \alpha^{\vee}\right\rangle \in \mathbb{N},
$$

then $f$ is integrable on $L(\Lambda)$.

Proof. Due to Proposition 1.1, Proposition 1.2 holds if $S=\emptyset$. Let $N=\left\langle\Lambda, \alpha^{\vee}\right\rangle$. It is well-known (cf. [K3]) that $f$ is integrable on $L(\Lambda)$ iff

$$
f^{N+1} v_{\Lambda} \text { lies in a maximal submodule of the Verma module } M(\Lambda) .
$$

But we have just shown that (1.5) holds for a Zariski open set of $\lambda$ on the hyperplane $\left\langle\lambda, \alpha^{\vee}\right\rangle=$ $N$. Since (1.5) is a polynomial condition, we conclude that it holds for all $\lambda$ on this hyperplane.

Proposition 1.3. If, under the assumptions of Proposition 1.1, $f$ is integrable on $L(\Lambda)$ and

$$
\left\langle\Lambda+\rho, \beta_{i}^{\vee}\right\rangle \neq 0 \text { for } i=0,1, \ldots, s(\leq k),
$$

then $\left\langle\Lambda-\sum_{i=0}^{s} \beta_{i}, \alpha^{\vee}\right\rangle \in \mathbb{Z}_{+}$. 
Proof. We have: $\left\langle\Lambda, \beta_{0}^{\vee}\right\rangle=\left\langle\Lambda+\rho, \beta_{0}^{\vee}\right\rangle \neq 0$, hence, by (1.4) we have: $\Lambda+\rho=\Lambda^{(1)}+\rho^{(1)}$, etc. Thus, $\Lambda^{(i)}+\rho^{(i)}=\Lambda+\rho$ for $i=1, \ldots, s$. Therefore, by Lemma 1.4 $\mathrm{b}$, we have:

$$
\Lambda^{(s)}=\Lambda-\sum_{i=0}^{s} \beta_{i} .
$$

Now the proposition follows from Lemma 1.5a.

The calculation of coroots is facilitated by the following simple fact.

Proposition 1.4. (a) There exists a non-degenerate symmetric bilinear form (.|.) on $\mathfrak{h}$ such that, identifying $\mathfrak{h}$ and $\mathfrak{h}^{*}$ via this form, we have:

$$
\alpha_{i}^{\vee}=\nu_{i} \alpha_{i} \text {, where } \nu_{i} \in \mathbb{C}^{\times},
$$

if and only if

$$
A=\operatorname{diag}\left(\nu_{i}\right)_{i \in I} B \text {, where } B=\left(b_{i j}\right) \text { is a symmetric matrix. }
$$

One then has: $\left(\alpha_{i} \mid \alpha_{j}\right)=b_{i j}$.

(b) Let $\Pi^{\vee \prime}=\left\{\alpha_{i}^{\vee \prime}\right\}=r_{s}\left(\Pi^{\vee}\right)$ and $\Pi=\left\{\alpha_{i}^{\prime}\right\}=r_{s}(\Pi)$ where $r_{s}$ is an odd reflection, and suppose that (1.0) holds. Then

$$
\alpha_{i}^{\vee \prime}=\nu_{i} \alpha_{i}^{\prime}
$$

(c) Provided that (1.7) holds and $a_{i i}=2$ or 0 for all $i \in I$, one has for any non-isotropic root $\alpha$ which is obtained from a simple root by a sequence of odd reflections: $\alpha^{\vee}=2 \alpha /(\alpha \mid \alpha)$.

Proof. (a) is proved in [K3], (b) and (c) are easily checked.

Remark 1.1. A natural question is which of the Lie superalgebras $\mathfrak{g}(\mathcal{D})$ are of "Kac-Moody" type? The most natural answer, in our opinion, is that they should satisfy the following conditions:

(i) $\mathfrak{g}(\mathcal{D})_{\overline{0}}$ is a (generalized) Kac-Moody algebra,

(ii) the $\mathfrak{g}(\mathcal{D})_{\overline{0}}$-module $\mathfrak{g}(\mathcal{D})_{\overline{1}}$ is integrable.

This definition covers the basic classical finite-dimensional Lie superalgebras and the associated affine superalgebras (including the twisted ones). Unfortunately, a well developed theory of generalized Kac-Moody superalgebras (see [B], [R] and references there) does not cover most of the latter superalgebras (because of the crucial assumption on the Cartan matrix that its off diagonal entries are non-positive). 


\section{Classification of integrable irreducible highest weight modules over $g \ell(m \mid n)^{\curvearrowleft}$}

Consider the Lie superalgebra $g \ell(m \mid n)$, where $m, n \geq 1$ (see [K1]). Let $e_{i j}(1 \leq i, j \leq m+n)$ denote its standard basis. Denote by $\mathfrak{h}$ the Cartan subalgebra of $g \ell(m \mid n)$ consisting of all diagonal matrices. Let $\epsilon_{i}(1 \leq i \leq m+n)$ be the basis of $\mathfrak{h}^{*}$ dual to the basis $u_{i}:=e_{i i}$ of $\mathfrak{h}$. Then $g \ell(m \mid n)=\mathfrak{g}(\mathcal{D})$ for the following data $\mathcal{D}=\left\{\mathfrak{h}, I, I_{1}, \Pi^{\vee}, \Pi\right\}$ (cf. [K1]). We let $I=\{1,2, \ldots, m+n-1\}, I_{1}=\{m\} ; \alpha_{i}^{\vee}=u_{i}-u_{i+1}$ for $i \in I \backslash I_{1}, \alpha_{m}^{\vee}=u_{m}+u_{m+1}$, $\alpha_{i}=\epsilon_{i}-\epsilon_{i+1}$ for all $i \in I$. Its Cartan matrix is the following $(m+n-1) \times(m+n-1)$ matrix:

$$
A=\left(\begin{array}{rrrrrrrr}
2 & -1 & 0 & & & & & \\
-1 & 2 & -1 & & & & & \\
& & \ddots & & & & & \\
& & -1 & 2 & -1 & & & \\
& & & -1 & 0 & 1 & & \\
& & & & -1 & 2 & -1 & \\
& & & & & \ddots & & \\
& & & & & & -1 & 2
\end{array}\right) \text { m-th row. }
$$

The Chevalley generators are as follows:

$$
e_{i}=e_{i, i+1}, f_{i}=e_{i+1, i} \quad(i=1, \ldots, m+n-1) .
$$

Note that $\alpha_{m}$ is the only odd simple root, and it is isotropic.

Consider the supertrace form on $g \ell(m \mid n)$ :

$$
(a \mid b)=\operatorname{str} a b .
$$

This is a non-degenerate invariant supersymmetric bilinear form on $g \ell(m \mid n)$ whose restriction to $\mathfrak{h}$ is non-degenerate and symmetric. Identifying $\mathfrak{h}$ and $\mathfrak{h}^{*}$ via this bilinear form, we have:

$$
\epsilon_{i}=u_{i} \text { for } i=1, \ldots, m ; \epsilon_{i}=-u_{i} \text { for } i=m+1, \ldots, m+n \text {. }
$$

Hence we have:

$$
\alpha_{i}^{\vee}=\alpha_{i} \text { for } i=1, \ldots, m, \quad \alpha_{i}^{\vee}=-\alpha_{i} \text { for } i=m+1, \ldots, m+n-1,
$$

and we may use Proposition 1.4. In particular,

$$
\left(\left(\alpha_{i} \mid \alpha_{i}\right)\right)_{i, j \in I}=\operatorname{diag}(\underbrace{1, \ldots, 1}_{m},-1, \ldots,-1) A .
$$

Likewise, the affine superalgebra $g \ell(m \mid n)$ is isomorphic to $\mathfrak{g}(\widehat{\mathcal{D}})$, where the data $\widehat{\mathcal{D}}=$ $\left\{\widehat{\mathfrak{h}}, \widehat{I}, \widehat{I}_{1}, \widehat{\Pi}^{\vee}, \widehat{\Pi}\right\}$ is an extension of the data $\mathcal{D}$ for $g \ell(m \mid n)$ defined as follows (cf. [K3]). The space $\widehat{\mathfrak{h}}$ is defined by (0.4), $\widehat{I}=I \cup\{0\}, \widehat{I_{1}}=\{m, 0\}, \widehat{\Pi}^{\vee}=\Pi^{\vee} \cup\left\{\alpha_{0}^{\vee}\right\}, \widehat{\Pi}=\Pi \cup\left\{\alpha_{0}\right\}$. Here the $\alpha_{i}$ for $i \in \Pi$ are extended from $\mathfrak{h}$ to $\widehat{\mathfrak{h}}$ by letting $\alpha_{i}(K)=\alpha_{i}(d)=0, \alpha_{0}=\delta-\theta, \alpha_{0}^{\vee}=K-\theta^{\vee}$, where: $\left.\delta\right|_{\mathfrak{h}+\mathbb{C} K}=0,\langle\delta, d\rangle=1, \theta=\epsilon_{1}-\epsilon_{m+n}$ is the highest root of $g \ell(m \mid n), \theta^{\vee}=u_{1}+u_{m+n}$. 
We extend the bilinear form (.|.) from $g \ell(m \mid n)$ to $g \ell(m \mid n) \widehat{\text { by }}(0.3)$. Identifying $\widehat{\mathfrak{h}}$ with $\widehat{\mathfrak{h}}^{*}$ via this symmetric bilinear form, we get:

$$
K=\delta, \quad \theta=\theta^{\vee}, \quad \alpha_{0}=\alpha_{0}^{\vee}
$$

We have the following expression of $\delta=K$ in terms of simple roots and coroots:

$$
\delta=K=\sum_{i=0}^{m+n-1} \alpha_{i}=\sum_{i=0}^{m} \alpha_{i}^{\vee}-\sum_{j=m+1}^{m+n-1} \alpha_{j}^{\vee} .
$$

The Cartan matrix for $\widehat{\mathcal{D}}$ is

$$
\widehat{A}=\left(\begin{array}{rrrrrr}
0 & -1 & 0 & \cdots & 0 & 1 \\
-1 & & & & & \\
0 & & & & & \\
\cdots & & & A & & \\
0 & & & & & \\
-1 & & & & &
\end{array}\right) .
$$

As above, we have:

$$
\left(\left(\alpha_{i} \mid \alpha_{j}\right)\right)_{i, j \in \widehat{I}}=\operatorname{diag}(\underbrace{1, \ldots, 1}_{m+1},-1, \ldots,-1) \widehat{A} .
$$

The even part of $g \ell(m \mid n)$ is $g \ell(m) \oplus g \ell(n)$, hence the even part of $g \ell(m \mid n)$ is the sum $g \ell(m) \hat{)}+g \ell(n)$ with a common central element $K$ and a common scaling element $d$. Note that the restriction of the supertrace form to $g \ell(m)$ (resp. $g \ell(n)$ ) is the normalized (resp. negative of the normalized) invariant form, i.e., $(\alpha \mid \alpha)=2$ (resp. $(\alpha \mid \alpha)=-2)$ for any root $\alpha$.

The set of simple roots for $g \ell(m) \hat{~(r e s p . ~} g \ell(n) \hat{)}$ ) is empty if $m=1$ (resp. $n=1$ ), and for $m \geq 2$ (resp. $n \geq 2$ ) it is as follows:

$$
\widehat{\Pi}^{\prime}=\left\{\alpha_{0}^{\prime}=\delta-\theta^{\prime}, \alpha_{1}, \ldots, \alpha_{m-1}\right\}
$$

(resp. $\widehat{\Pi}^{\prime \prime}=\left\{\alpha_{0}^{\prime \prime}=\delta-\theta^{\prime \prime}, \alpha_{m+1}, \ldots, \alpha_{m+n-1}\right)$, where $\theta^{\prime}=\sum_{i=1}^{m-1} \alpha_{i}, \theta^{\prime \prime}=\sum_{i=m+1}^{m+n-1} \alpha_{i}$. Assuming that $m \geq 2$, we have: $\left(\theta^{\prime} \mid \theta^{\prime}\right)=2$, hence $\theta^{\prime}=\theta^{\prime} \vee$, and we have:

$$
\alpha_{0}^{\prime}=\alpha_{0}^{\prime \vee}=\alpha_{0}+\sum_{i=m}^{m+n-1} \alpha_{i}=\alpha_{0}^{\vee}+\alpha_{m}^{\vee}-\sum_{i=m+n}^{m+n-1} \alpha_{i}^{\vee}
$$

A $g \ell(m \mid n)$-module $L(\Lambda)$ is called integrable, if its restriction to $g \ell(m)$ is integrable and its restriction to $g \ell(m \mid n)$ is locally finite. In this section we shall classify all such modules.

As usual, define fundamental weights $\Lambda_{i} \in \widehat{\mathfrak{h}} \quad(i=0,1, \ldots, m+n-1)$ by

$$
\left\langle\Lambda_{i}, \alpha_{j}^{\vee}\right\rangle=\delta_{i j}, j=0, \ldots, m+n-1,\left\langle\Lambda_{i}, d\right\rangle=0,
$$


and labels of a weight $\Lambda$ by:

$$
k_{i}=\left\langle\Lambda, \alpha_{i}^{\vee}\right\rangle .
$$

The following necessary conditions of integrability of $L(\Lambda)$ follow from Lemma 1.5a:

$$
\begin{array}{r}
k_{i} \in \mathbb{Z}_{+} \text {for } i=1, \ldots, m-1, m+1, \ldots, m+n-1, \\
k^{\prime}:=k_{0}+k_{m}-\sum_{i=m+1}^{m+n-1} k_{i} \in \mathbb{Z}_{+} .
\end{array}
$$

We assume in (2.6) that $m \geq 2$ and use (2.4).

We call $k^{\prime}$ the partial level of $\Lambda$ since, using (2.3), we see that the level $k:=\langle\Lambda, K\rangle$ is given by

$$
k=\sum_{i=1}^{m-1} k_{i}+k^{\prime} .
$$

Hence, provided that $m \geq 2$, the level of an integrable $g \ell(m \mid n)$-module is a non-negative integer.

Lemma 2.1. Assume that $m \geq 2$. Then conditions (2.5) and (2.9) along with the condition

$$
k^{\prime} \geq n
$$

are sufficient for integrability of the $g \ell(m \mid n)$-module $L(\Lambda)$.

Proof. The lemma follows from Lemma 1.5 applied to the simple roots $\alpha_{i}, i=1, \ldots, m-1$, and Proposition 1.2 applied to $\alpha^{\vee}=\alpha_{0}^{\prime \vee}$, since, due to (2.4) we have:

$$
\left\langle\rho, \alpha_{0}^{\prime \vee}\right\rangle=-n+1 \text {. }
$$

Lemma 2.2. Let $L(\Lambda)$ be an integrable $g \ell(m \mid n)$-module such that $k^{\prime}<n$, and let $m \geq 2$. Then the following complementary condition holds:

$$
\text { there exist } r, s \in \mathbb{Z}_{+} \text {such that }
$$

(i) $k^{\prime}=r+s$,

(ii) $k_{0}-k_{m+n-1}-k_{m+n-2}-\cdots-k_{m+n-r}-r=0$,

(iii) $k_{m}-k_{m+1}-k_{m+2}-\cdots-k_{m+s}-s=0$.

Proof. Consider the following two sequences of roots of $g \ell(m \mid n) \hat{\text { : }}$

$\beta_{0}=\alpha_{0}, \beta_{1}=\alpha_{0}+\alpha_{m+n-1}, \beta_{2}=\alpha_{0}+\alpha_{m+n-1}+\alpha_{m+n-2}, \ldots, \beta_{n-1}=\alpha_{0}+\alpha_{m+n-1}+\ldots+\alpha_{m+1} ;$

$\beta_{0}^{\prime}=\alpha_{m}, \beta_{1}^{\prime}=\alpha_{m}+\alpha_{m+1}, \beta_{2}^{\prime}=\alpha_{m}+\alpha_{m+1}+\alpha_{m+2}, \ldots, \beta_{n-1}^{\prime}=\alpha_{m}+\cdots+\alpha_{m+n-1}$. 
It is clear by Proposition 1.4 that $\beta_{i}^{\vee}=\beta_{i}$ and $\beta^{\prime} \vee=\beta_{i}^{\prime}$. Note that $\left\langle\Lambda+\rho, \beta_{r}^{\vee}\right\rangle\left(\operatorname{resp} .\left\langle\Lambda+\rho, \beta_{s}^{\prime} \vee\right\rangle\right)$ is equal to the left-hand side of (ii) (resp. (iii)). Note that

$$
\left\langle\beta_{i}, \alpha_{0}^{\prime \vee}\right\rangle=1=\left\langle\beta_{i}^{\prime}, \alpha_{0}^{\prime \vee}\right\rangle, i=0, \ldots, n-1 .
$$

If $\left\langle\Lambda+\rho, \beta_{i}^{\vee}\right\rangle \neq 0$ for all $i$, using (2.6) and (2.10) we would conclude, by Proposition 1.3, that $k^{\prime}-n \geq 0$, in contradiction with the assumption of the lemma. Hence (ii) holds for some nonnegative integer $r(<n)$. Similarly, (iii) holds for some non-negative integer $s(<n)$. Similarly, applying Proposition 1.3 to the union of sequences $\beta_{i}$ and $\beta_{i}^{\prime}$, we conclude that

$$
r+s \leq k^{\prime}
$$

Hence, adding up (ii) and (iii) we get

$$
k^{\prime}+\sum_{i=m+s+1}^{m+n-r-1} k_{i}=r+s .
$$

Now (i) follows from (2.5), (2.11) and (2.12).

Remark 2.1. Condition $(*)$ on $\Lambda$ is equivalent to the following condition: there exists a nonnegative integer $s \leq k^{\prime}(\leq n-1)$ such that:

$$
k_{m}=k_{m+1}+\cdots+k_{m+s}+s \text { and } k_{m+s+1}=\cdots=k_{m+s+n-k^{\prime}-1}=0 .
$$

This condition implies that $\Lambda$ lies in a union of $k^{\prime}+1$ hyperplanes of dimension $k^{\prime}+m-1$. Equivalently, there exists a non-negative integer $r \leq k^{\prime}(\leq n-1)$ such that

$$
k_{0}=k_{m+n-1}+k_{m+n-2}+\cdots+k_{m+n-r}+r \text { and } k_{m+n-r-1}=\cdots=k_{m+k^{\prime}-r+1}=0 .
$$

Theorem 2.1. (a) A $g \ell(1 \mid n)$-module $L(\Lambda)$ is integrable iff $k_{2}, \ldots, k_{n} \in \mathbb{Z}_{+}$.

(b) Provided that $m \geq 2$, a $g \ell(m \mid n)$-module $L(\Lambda)$ is integrable iff conditions (2.5), (2.6) hold and, in the case $k^{\prime}<n$, the complementary condition $\left({ }^{*}\right)$ holds.

Proof. In the case $m=1$, the only condition of integrability is local finiteness of $g \ell(1, n)$ on $L(\Lambda)$ which is equivalent to $k_{2}, \ldots, k_{n} \in \mathbb{Z}_{+}$due to Lemma 1.5b. It follows from Lemma 2.2 that in the case $m \geq 2$, the conditions listed by Theorem 2.1 b are necessary. In view of Lemma 1.5b, it remains to show that these conditions are sufficient for local nilpotency of $e_{-\alpha_{0}^{\prime}}$. Due to Lemma 2.1, we may assume that

$$
k^{\prime} \leq n-1 .
$$

Consider the sequence of odd roots $\beta_{0}, \ldots, \beta_{n-1}$ introduced in the proof of Lemma 2.2 and let $\Pi^{(0)}=\Pi, \Pi^{(1)}=r_{\beta_{0}}\left(\Pi^{(0)}\right), \ldots, \Pi^{(n)}=r_{\beta_{n-1}}\left(\Pi^{(n-1)}\right)$, and notice that

$$
\alpha_{0}^{\prime} \in \Pi^{(n)} .
$$


Let $\Lambda^{(n)}$ be the highest weight vector of $L(\Lambda)$ with respect to $\mathfrak{n}_{+}^{(n)}=r_{\beta_{n-1}} \ldots r_{\beta_{0}}(\mathfrak{n})$. Due to Lemma 1.5 b, it remains to show that conditions listed by Theorem 2.1 b imply that

$$
\left\langle\Lambda^{(n)}, \alpha_{0}^{\prime}\right\rangle \in \mathbb{Z}_{+} \text {. }
$$

Recall that by (1.4) we have:

$$
\Lambda^{(n)}+\rho^{(n)}=\Lambda+\rho+\sum_{i \in S} \beta_{i}
$$

where $S=\left\{i \in[0, \ldots, n-1] \mid\left\langle\Lambda^{(i)}, \beta_{i}^{\bigvee}\right\rangle=0\right\}$. Let $t_{i}=\left\langle\Lambda^{(i)}, \beta_{i}^{\bigvee}\right\rangle$ for short. Then condition (*) gives for some $r \in \mathbb{Z}_{+}, r<n$, that $t_{r}=0$. In view of Remark 2.1, we have:

$$
t_{r}=t_{r+1}=\cdots=t_{n-s-1}=0 .
$$

Hence, due to (2.15), (2.9), (2.10) and (2.16) we get:

$$
\left\langle\Lambda^{(n)}+\rho^{(n)}, \alpha_{0}^{\prime} \vee\right\rangle=k^{\prime}+(1-n)+|S| \geq k^{\prime}+(1-n)+(n-s-r) \geq 1,
$$

proving (2.14), since $\left\langle\rho^{(n)}, \alpha_{0}^{\prime \vee}\right\rangle=1$.

Remark 2.2. It follows from Theorem 2.1 that when $m \geq 2$, the only integrable $g \ell(m \mid n)$ modules $L(\Lambda)$ of level $k=0$ are those for which all labels are 0 , in which case $\operatorname{dim} L(\Lambda)=1$.

Remark 2.3. If $m \geq 2$ and $n \geq 2$, then the only $L(\Lambda)$ which are integrable with respect to the whole even subalgebra are 1-dimensional. (It is because the $g \ell(m)$-integrability implies $k \geq 0$ and $g \ell(n)$-integrability implies $k \leq 0$.)

Remark 2.4. Define $\epsilon \in \widehat{\mathfrak{h}}^{*}$ by letting $\left.\epsilon\right|_{\mathfrak{h}}=$ supertrace, $\epsilon(K)=\epsilon(d)=0$. It follows from Theorem 2.1 that when $m \geq 2$, the complete list of highest weights of integrable $g \ell(m \mid n)$ modules of level 1, up to adding an arbitrary linear combination of $\epsilon$ and $\delta$, is as follows:

$$
\Lambda_{s}(1 \leq s \leq m-1), \quad(a+1) \Lambda_{m}+a \Lambda_{m+1}\left(a \in \mathbb{Z}_{+}\right) \quad(a+1) \Lambda_{0}+a \Lambda_{m+n-1}\left(a \in \mathbb{Z}_{+}\right) .
$$

Remark 2.5. Consider the sequence of the sets of simple roots $\Pi^{(0)}=\Pi, \ldots, \Pi^{(n)}=$ $\left\{\alpha_{0}^{\prime}, \ldots, \alpha_{m+n-1}^{\prime}\right\}$, introduced in the proof of Theorem 2.1. One has:

$$
\begin{aligned}
\alpha_{0}^{\prime} & =\alpha_{0}+\alpha_{1}, \alpha_{1}^{\prime}=\alpha_{2}, \ldots, \alpha_{m-2}^{\prime}=\alpha_{m-1}, \alpha_{m-1}^{\prime}=\alpha_{m}+\alpha_{m+1}+\cdots+\alpha_{m+n-1}+\alpha_{0}, \\
\alpha_{m}^{\prime} & =-\left(\alpha_{m+n}+\cdots+\alpha_{m+n-1}+\alpha_{0}\right), \alpha_{j}^{\prime}=\alpha_{j} \text { for } m+1 \leq j \leq m+n-1 .
\end{aligned}
$$

Let $\Lambda_{j}^{\prime}$ be the fundamental weights with respect to $\Pi^{(n)}$. Given a weight $\Lambda$, denote by $\Lambda^{(n)}$ the highest weight of $L(\Lambda)$ with respect to $\Pi^{(n)}$ (or rather $\mathfrak{n}_{+}^{(n)}$ ). Using Lemma 1.4, it is easy to see that the weights listed in Remark 2.4 get changed under the map $\Lambda \mapsto \Lambda^{(n)}$ as follows:

$$
\begin{aligned}
\Lambda_{j}^{(n)}= & \Lambda_{j} \quad(1 \leq j \leq m),\left((a+1) \Lambda_{0}+a \Lambda_{m+n-1}\right)^{(n)}=(a+1) \Lambda_{0}+a \Lambda_{m+n-1}-\alpha_{0}, \\
& \left((a+1) \Lambda_{m}+a \Lambda_{m+1}\right)^{(n)}=(a+1) \Lambda_{m}+a \Lambda_{m+1}+\alpha_{m}^{\prime}(a>0) .
\end{aligned}
$$

In terms of the fundamental weights $\Lambda_{j}^{\prime}$ the map $\Lambda \rightarrow \Lambda^{(n)}$ looks as follows:

$$
\begin{aligned}
& \Lambda_{j} \mapsto \Lambda_{j-1}^{\prime} \quad(1 \leq j \leq m), \\
& (a+1) \Lambda_{0}+\Lambda_{m+n-1} \mapsto(a+2) \Lambda_{0}^{\prime}+(a+1) \Lambda_{m+n-1}^{\prime}\left(a \in \mathbb{Z}_{+}\right), \\
& (a+1) \Lambda_{m}+a \Lambda_{m+1} \mapsto a \Lambda_{m}^{\prime}+(a-1) \Lambda_{m+1}^{\prime} \quad(a \in \mathbb{N}) .
\end{aligned}
$$

It follows that all weights of level 1 listed by Remark 2.4 are conjugate to each other by odd reflections. 


\section{Free field realization of level 1 integrable modules over $g \ell(m \mid n)$.}

Fix non-negative integers $m$ and $n$ such that $m+n \geq 1$ and denote by $F$ the vertex algebra generated by $m$ pairs of odd fields $\psi^{i}(z), \psi^{i *}(z),(i=1, \ldots, m)$ and $n$ pairs of even fields $\varphi^{j}(z), \varphi^{j *}(z)(j=1, \ldots, n)$, all pairwise local, subject to the following operator product expansions (as usual, we list only the non-trivial OPE):

$$
\begin{array}{ll}
\psi^{i}(z) \psi^{j *}(w) \sim \frac{\delta_{i j}}{z-w}, & \psi^{i *}(z) \psi^{j}(w) \sim \frac{\delta_{i j}}{z-w}, \\
\varphi^{i}(z) \varphi^{j *}(w) \sim-\frac{\delta_{i j}}{z-w}, & \varphi^{i *}(z) \varphi^{j}(w) \sim \frac{\delta_{i j}}{z-w} .
\end{array}
$$

This is called a free fermionic vertex algebra in the book [K4] to which we refer for foundations of the vertex algebra theory.

This vertex algebra has a family of Virasoro fields [K4], from which it is convenient to choose the following one:

$$
\begin{aligned}
L(z) \equiv \sum_{k \in \mathbb{Z}} L_{k} z^{-k-2} & =\frac{1}{2} \sum_{i=1}^{m}\left(: \partial \psi^{i}(z) \psi^{i *}(z):+: \partial \psi^{i *}(z) \psi^{i}(z):\right) \\
& +\frac{1}{2} \sum_{j=1}^{n}\left(: \partial \varphi^{j}(z) \varphi^{j *}(z):-: \partial \varphi^{j *}(z) \varphi^{j}(z):\right) .
\end{aligned}
$$

With respect to $L(z)$ the fields $\psi^{i}(z), \psi^{i *}(z), \varphi^{j}(z)$ and $\varphi^{j *}(z)$ are primary of conformal weight $1 / 2$. We therefore write all these fields in the form $x^{i}(z)=\sum_{k \in \frac{1}{2}+\mathbb{Z}} x_{k}^{(i)} z^{-k-1 / 2}$ where $x=\psi$, $\psi^{*}, \varphi$ or $\varphi^{*}$, and we have the following conditions on the vacuum $|0\rangle$ :

$$
\psi_{k}^{(i)}|0\rangle=0, \quad \psi_{k}^{(i) *}|0\rangle=0, \quad \varphi_{k}^{(i)}|0\rangle=0, \quad \varphi_{k}^{(i) *}|0\rangle=0 \text { for } k>0 .
$$

The operator $L_{0}$ is called the energy operator or (Hamiltonian) and its eigenvalues are called the energies of the corresponding eigenvectors. The energy can be calculated from the following relations:

$$
\text { energy }|0\rangle=0, \quad \operatorname{energy}\left(\psi_{k}^{(i)}, \psi_{k}^{(i) *}, \varphi_{k}^{(j)}, \varphi_{k}^{(j) *}\right)=-k \text {. }
$$

The second relation means that $\psi_{k}^{(i)}$, etc., changes the energy by $-k$, i.e.,

$$
\operatorname{energy}\left(\psi_{k}^{(i)} v\right)=\operatorname{energy}(v)-k, \text { etc. }
$$

Next, for each pair $i, j$ that may occur introduce the following fields of conformal weight 1 :

$$
\begin{array}{ll}
a^{i j+}(z)=: \psi^{i}(z) \psi^{j *}(z):, & a^{i j-}(z)=: \varphi^{i}(z) \varphi^{j *}(z): \\
E^{i j+}(z)=: \psi^{i}(z) \varphi^{j *}(z):, & E^{i j-}(z)=: \varphi^{i}(z) \psi^{j *}(z): .
\end{array}
$$

Proposition 3.1. (a) Consider the affine superalgebra $g \ell(m \mid n)$ and let $A(z)=$ $\sum_{k \in \mathbb{Z}}\left(t^{k} \otimes A\right) z^{-k-1}$ for $A \in g \ell(m \mid n)$. Then the linear map $\sigma$ given by

$$
\begin{array}{ll}
e_{i j}(z) \mapsto a^{i j+}(z), & e_{i+m, j+m}(z) \mapsto a^{i j-}(z), \\
e_{i, j+m}(z) \mapsto E^{i j+}(z), & e_{i+m, j}(z) \mapsto E^{i j-}(z), \quad K \mapsto 1, \quad d \mapsto L_{0}
\end{array}
$$

defines a representation of $g \ell(m \mid n)$ (of level 1$)$ in the space $F$. 
(b) Consider the standard $g \ell(m \mid n)$-module $\mathbb{C}^{m \mid n}$ and its contragredient module $\mathbb{C}^{m \mid n *}$. Consider the corresponding $\mathbb{C}\left[t, t^{-1}\right] \otimes g \ell(m \mid n)$-modules $\mathbb{C}\left[t, t^{-1}\right] \otimes \mathbb{C}^{m \mid n}$ and $\mathbb{C}\left[t, t^{-1}\right] \otimes \mathbb{C}^{m \mid n *}$, and let $v(z)=\sum_{k \in \mathbb{Z}}\left(t^{k} \otimes v\right) z^{-k-1}$ for $v \in \mathbb{C}^{m \mid n}$ or $\mathbb{C}^{m \mid n *}$. Then the linear maps $\nu$ and $\nu^{*}$ given by $(i=1, \ldots, m ; j=1, \ldots, n)$ :

$$
\begin{array}{ll}
v_{i}(z) \mapsto \psi^{i}(z), & v_{j+m}(z) \mapsto \varphi^{j}(z) \text { and } \\
v_{i}^{*}(z) \mapsto \psi^{i *}(z), & v_{j+m}^{*}(z) \mapsto \varphi^{j *}(z)
\end{array}
$$

are equivariant, i.e., they have the following property:

$$
\begin{aligned}
\nu(A(z) v(w)) & =[\sigma(A(z)), \nu(v(w))], \quad v \in \mathbb{C}^{m \mid n} \\
\nu^{*}\left(A(z) v^{*}(w)\right) & =\left[\sigma(A(z)), \nu^{*}\left(v^{*}(w)\right)\right], \quad v^{*} \in \mathbb{C}^{m \mid n} .
\end{aligned}
$$

Proof. Both statements follow from the corresponding OPE, which are easily derived from Wick's formula. Below we give the less trivial OPE needed for the proof of (a).

$$
\begin{aligned}
E^{i j+}(z) E^{k \ell-}(w) & \sim \frac{\delta_{j k} a^{i \ell+}(w)+\delta_{i \ell} a^{k j-}(w)}{z-w}+\frac{\delta_{i \ell} \delta_{j k}}{(z-w)^{2}} \\
a^{i j \pm}(z) E^{k \ell \pm}(w) & \sim \frac{\delta_{j k} E^{i \ell \pm}(w)}{z-w}, \\
a^{i j \pm}(z) E^{k \ell \mp}(w) & \sim \frac{-\delta_{\ell i} E^{k j \mp}(w)}{z-w}, \\
a^{i j \pm}(z) a^{k \ell \pm}(w) & \sim \frac{\delta_{j k} a^{i \ell \pm}(w)-\delta_{\ell i} a^{k j \pm}(w)}{z-w} \pm \frac{1}{(z-w)^{2}} \\
a^{i j \pm}(z) a^{k \ell \mp}(w) & \sim 0 .
\end{aligned}
$$

Introduce the total charge operator

$$
a_{0}=\sigma(I), \text { where } I=\sum_{i=1}^{m+n} e_{i i} \in g \ell(m \mid n) .
$$

Its eigenvalues are called charges of the corresponding eigenvectors. It is clear from Proposition 3.1 that the total charge can be calculated from the following relations:

$$
\text { charge }|0\rangle=0, \quad \text { charge }\left(\psi_{k}^{(i)}, \varphi_{k}^{(j)}\right)=1, \quad \operatorname{charge}\left(\psi_{k}^{(i) *}, \varphi_{k}^{(j) *}\right)=-1 .
$$

Consider the charge decomposition of $F$, i.e., its decomposition in eigenspaces of $a_{0}$ :

$$
F=\oplus_{s \in \mathbb{Z}} F_{s} .
$$

Since $a_{0}$ commutes with $\sigma(g \ell(m \mid n) \hat{)}$, we conclude that (3.4) is a decomposition in a direct sum of $g \ell(m \mid n)$-modules. 
It is clear that $L_{0}$ commutes with $a_{0}$, hence each $F_{s}$ is $L_{0}$-invariant, and since all eigenvalues of $L_{0}$ in $F$ lie in $\frac{1}{2} \mathbb{Z}_{+}$, the same holds for eigenvalues of $L_{0}$ in $F_{s}, s \in \mathbb{Z}$. Note also that $L_{0}$ commutes with $\sigma(g \ell(m \mid n))$. It is because all fields $a^{i j \pm}(z)$ and $E^{i j \pm}(z)$ have conformal weight 1. It follows that each eigenspace of $L_{0}$ in $F_{s}$ is a $g \ell(m \mid n)$-module. The following proposition describes the lowest energy subspace $F_{s}^{\text {low }}$ and the lowest weight vector $|s\rangle$ in each $F_{s}$.

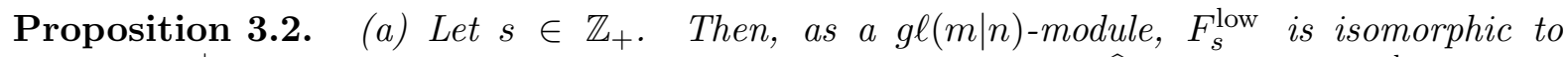
$\Lambda^{s} \mathbb{C}^{m \mid n}$. Furthermore, any highest weight vector of $g \ell(m \mid n)$ in $F_{s}$ lies in $F_{s}^{\text {low }}$ and is proportional to the vector

$$
|s\rangle=\psi_{-\frac{1}{2}}^{(1)} \ldots \psi_{-\frac{1}{2}}^{(s)}|0\rangle \text { with weight } \Lambda_{0}+\epsilon_{1}+\cdots+\epsilon_{s}-\frac{s}{2} \delta
$$

provided that $s \leq m$, and to the vector

$$
|s\rangle=\left(\varphi_{-\frac{1}{2}}^{(1)}\right)^{s-m}|m\rangle \text { with weight } \Lambda_{0}+\epsilon_{1}+\cdots+\epsilon_{m}+(s-m) \epsilon_{m+1}-\frac{s}{2} \delta
$$

provided that $s \geq m$.

(b) Let $-s \in \mathbb{Z}_{+}$. Then, as a $g \ell(m \mid n)$-module, $F_{s}^{\text {low }}$ is isomorphic to $\Lambda^{-s}\left(\mathbb{C}^{m \mid n}\right)^{*}$. Furthermore, any highest weight vector of $g \ell(m \mid n)$ in $F_{s}$ lies in $F_{s}^{\text {low }}$ and is proportional to the vector

$$
|s\rangle=\left(\varphi_{-\frac{1}{2}}^{(n) *}\right)^{-s}|0\rangle \text { with weight } \Lambda_{0}+s \epsilon_{m+n}+\frac{s}{2} \delta
$$

Proof. It is clear that, if $s$ (resp. $-s) \in \mathbb{Z}_{+}$, then $F_{s}^{\text {low }}$ consists of homogeneous polynomials of degree $|s|$ in anticommuting operators $\psi_{-\frac{1}{2}}^{(i)}\left(\right.$ resp. $\left.\psi_{-\frac{1}{2}}^{(i) *}\right)$ and commuting operators $\varphi_{-\frac{1}{2}}^{(j)}$ (resp. $\varphi_{-\frac{1}{2}}^{(j) *}$, applied to $|0\rangle$. This proves (a) (resp. (b)), due to Proposition 3.1.

Remark 3.1. The lowest energy in $F_{s}$ is $\frac{1}{2}|s|$ and the spectrum of $L_{0}$ in $F_{s}$ is $\frac{1}{2}|s|+\mathbb{Z}_{+}$.

Remark 3.2. Denote by $\Lambda_{(s)}$ the weight of $|s\rangle$. When restricted to $s \ell(m \mid n) \hat{,}, \Lambda_{(s)}$ is given by the following formulas:

$$
\begin{array}{ll}
\Lambda_{s}-\frac{s}{2} \delta & \text { if } 0 \leq s \leq m, \\
(1+s-m) \Lambda_{m}+(s-m) \Lambda_{m+1}-\frac{s}{2} \delta & \text { if } s \geq m, \\
(1-s) \Lambda_{0}-s \Lambda_{m+n-1}+\frac{s}{2} \delta & \text { if } s \leq 0 .
\end{array}
$$

We identify here $\Lambda_{m+1}$ with $-\Lambda_{0}$ in the case $n=1$.

The following theorem is the central result of this section.

Theorem 3.1. Suppose that $m \geq 1$. Then each $g \ell(m \mid n)$-module $F_{s}, s \in \mathbb{Z}$, is an irreducible integrable highest weight module of level 1. 
Remark 3.3. The $g \ell(0 \mid n)$-modules $F_{s}$ are not irreducible. For example, one can show that in the case $(m, n)=(0,2)$, one has the following decomposition as $g \ell(2)$-modules (in the standard notation of [K3]):

$$
\operatorname{ch} F_{s}=\sum_{j=0}^{\infty} \operatorname{ch} L\left(-(1+2 j+|s|) \Lambda_{0}+(2 j+|s|) \Lambda_{1}\right) q^{j^{2}+(|s|+1) j+|s| / 2} .
$$

E. Frenkel informed one of us that he had found this decomposition too.

The proof of Theorem 3.1 is based on the (super) boson-fermion correspondence, which we shall now recall (cf. [K4]).

For each $i=1, \ldots, m$ there exists a unique invertible odd operator $e^{\epsilon_{i}}$ with inverse $e^{-\epsilon_{i}}$ satisfying the following three properties:

$$
\begin{aligned}
{\left[e^{\epsilon_{i}}, \psi^{j}(z)\right]=0 } & \text { if } i \neq j, \quad\left[e^{\epsilon_{i}}, \varphi^{j}(z)\right]=0 \text { for all } j, \\
e^{\epsilon_{i}} \psi_{k}^{(i)} e^{-\epsilon_{i}} & =\psi_{k-1}^{(i)}, \quad e^{\epsilon_{i}} \psi_{k}^{(i) *} e^{-\epsilon_{i}}=\psi_{k+1}^{(i) *}, \\
e^{\epsilon_{i}}|0\rangle & =\psi_{-\frac{1}{2}}^{(i)}|0\rangle, \quad e^{-\epsilon_{i}}|0\rangle=\psi_{-\frac{1}{2}}^{(i) *}|0\rangle .
\end{aligned}
$$

It is easy to see that $e^{\epsilon_{i}} e^{\epsilon_{j}}=-e^{\epsilon_{j}} e^{\epsilon_{i}}$ if $i \neq j$.

We let for short $(i=1, \ldots, m ; j=1, \ldots, n)$ :

$$
\epsilon^{i}(z)=a^{i i+}(z)=\sum_{k \in \mathbb{Z}} \epsilon_{k}^{(i)} z^{-k-1}, \quad \epsilon^{j+m}(z)=a^{j j-}(z)=\sum_{k \in \mathbb{Z}} \epsilon_{k}^{(j+m)} z^{-k-1} .
$$

Then we have:

$$
\left[\epsilon_{k}^{(i)}, e^{\epsilon_{j}}\right]=\delta_{i j} \delta_{k 0} e^{\epsilon_{j}}, \quad i=1, \ldots, m+n ; \quad j=1, \ldots, m .
$$

For each $i=1, \ldots, m+n$ introduce the following fields:

$$
\Gamma_{\epsilon_{i}}^{+}(z)=e^{\sum_{k=1}^{\infty} \frac{z^{k}}{k} \epsilon_{-k}^{(i)}}, \quad \Gamma_{\epsilon_{i}}^{-}(z)=e^{-\sum_{k=1}^{\infty} \frac{z^{-k}}{k} \epsilon_{k}^{(i)}},
$$

and for a linear combination with integer coefficients $\alpha=\sum_{i=1}^{m} s_{i} \epsilon_{i}$ we let $\Gamma_{\alpha}^{ \pm}(z)=\Pi_{i}\left(\Gamma_{\epsilon_{i}}^{ \pm}\right)^{s_{i}}$ (recall that all $\epsilon_{k}^{(i)}$ commute and all $\epsilon_{-k}^{(i)}$ commute for $k \geq 1$, see Proposition 3.1.a). The central fact of the classical boson-fermion correspondence is the following formula, see e.g. K4] $(i=1, \ldots, m)$

$$
\psi^{i}(z)=e^{\epsilon_{i}} z^{\epsilon_{0}^{(i)}} \Gamma_{\epsilon_{i}}^{+}(z) \Gamma_{\epsilon_{i}}^{-}(z), \quad \psi^{i *}(z)=e^{-\epsilon_{i}} z^{-\epsilon_{0}^{(i)}} \Gamma_{-\epsilon_{i}}^{+}(z) \Gamma_{-\epsilon_{i}}^{-}(z) .
$$

The key formulas of the super boson-fermion correspondence are the following [KL], K4] $(j=1, \ldots, n)$ :

$$
\begin{aligned}
\varphi^{j}(z) & =z^{\epsilon_{0}^{(i)}} e^{\epsilon_{i}} \Gamma_{\epsilon_{i}}^{+}(z) E^{j i-}(z) \Gamma_{\epsilon_{i}}^{-}(z) \\
\varphi^{j *}(z) & =z^{-\epsilon_{0}^{(i)}} e^{-\epsilon_{i}} \Gamma_{-\epsilon_{i}}^{+}(z) E^{i j+}(z) \Gamma_{-\epsilon_{i}}^{-}(z),
\end{aligned}
$$

for each $i=1, \ldots, m$ (we assume here that $m \geq 1$ ). 
Proof of Theorem 3.1. Since the eigenspaces of $L_{0}$ in $F_{s}$ are finite-dimensional and $L_{0}$ commutes with $g \ell(m \mid n)$, it follows that $F_{s}$ is a direct sum of finite-dimensional $g \ell(m \mid n)$-modules, hence $g \ell(m \mid n)$ acts locally finitely on $F_{s}$. Furthermore, we have:

$$
F=F^{\text {fermi }} \otimes F^{\text {bose }}
$$

where $F^{\text {fermi }}$ (resp. $F^{\text {bose }}$ ) is the vertex algebra generated by the $\psi^{i}(z), \psi^{i *}(z)$ (resp. $\varphi^{j}(z)$, $\left.\varphi^{j *}(z)\right)$, and the subalgebra $g \ell(m)$ of $g \ell(m \mid n)$ acts on $F$ via $\pi \otimes 1$, where the representation $\pi$

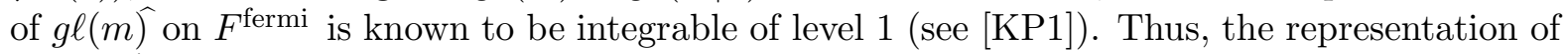
$g \ell(m \mid n)$ in each $F_{s}$ is integrable.

The irreducibility of $F_{s}$, provided that $m \geq 1$, is proved using (3.9) and (3.10) in exactly the same fashion as the proof of Theorem $5.8 \mathrm{a}$ from [K4].

Remark 3.4. We have got along the way the following vertex operator construction of $g \ell(m \mid n)$. For each $\alpha=\sum_{i=1}^{m} s_{i} \epsilon_{i}, s_{i} \in \mathbb{Z}$, introduce the usual vertex operator

$$
\Gamma_{\alpha}=e^{\alpha} z^{\alpha_{0}} \Gamma_{\alpha}^{+} \Gamma_{\alpha}^{-}
$$

Then the following map defines an irreducible integrable highest weight module of level 1 in each $F_{s}$ :

$$
\begin{array}{ll}
e_{i i}(z) \mapsto \epsilon_{i}(z) & (i=1, \ldots, m), \quad K \mapsto 1, \\
e_{i j}(z) \mapsto \Gamma_{\epsilon_{i}-\epsilon_{j}} & (i, j=1, \ldots, m), \\
e_{i+m, j+m}(z) \mapsto: \varphi^{i}(z) \varphi^{j *}(z): & (i, j=1, \ldots, n), \\
e_{j+m, i}(z) \mapsto \Gamma_{-\epsilon_{i}}(z) \varphi^{j}(z) & (i=1, \ldots, m ; j=1, \ldots, n), \\
e_{i, j+m}(z) \mapsto \Gamma_{\epsilon_{i}}(z) \varphi^{j *}(z) & (i=1, \ldots, m ; j=1, \ldots, n) .
\end{array}
$$

Next, we give a standard derivation of a "quasiparticle" character formula for the $g \ell(m \mid n \hat{)-}$ modules $F_{s}, s \in \mathbb{Z}$.

Given $a=\left(a_{1}, \ldots, a_{m}\right), b=\left(b_{1}, \ldots, b_{m}\right) \in \mathbb{Z}_{+}^{m}$ and $c=\left(c_{1}, \ldots, c_{n}\right), d=\left(d_{1}, \ldots, d_{n}\right) \in \mathbb{Z}_{+}^{n}$, denote by $F(a, b, c, d)$ the linear span of vectors in $F$ obtained from the vacuum vector $|0\rangle$ by applying all monomials in the $\psi_{k}^{(i)}, \psi_{k}^{(i) *}, \varphi_{k}^{(i)}, \varphi_{k}^{(i) *}$ which contain $a_{1}$ factors of the form $\psi_{k}^{(1)}$, $k \in \frac{1}{2}+\mathbb{Z}, \ldots, a_{m}$ factors of the form $\psi_{k}^{(m)}, b_{1}$ factors of the form $\psi_{k}^{(1) *}, \ldots, b_{m}$ factors of the form $\psi_{k}^{(m) *}, c_{1}$ factors of the form $\varphi_{k}^{(1)}, \ldots, d_{n}$ factors of the form $\varphi_{k}^{(n) *}$. These states lie in $F_{s}$ iff the following condition holds:

$$
|a|-|b|+|c|-|d|=s
$$

where $|a|=\sum a_{i}$, etc.

It is clear that the state of minimal energy in $F(a, b, c, d)$ is (up to a constant factor) the following vector:

$$
\begin{aligned}
v(a, b, c, d)= & \left(\psi_{-\left(a_{1}-\frac{1}{2}\right)}^{(1)} \ldots \psi_{-\frac{3}{2}}^{(1)} \psi_{-\frac{1}{2}}^{(1)}\right) \ldots\left(\psi_{-\left(a_{m}-\frac{1}{2}\right)}^{(m)} \ldots \psi_{-\frac{1}{2}}^{(m)}\right) \\
& \times\left(\psi_{-\left(b_{1}-\frac{1}{2}\right)}^{(1) *} \ldots \psi_{-\frac{1}{2}}^{(1) *}\right) \ldots\left(\psi_{-\left(b_{m}-\frac{1}{2}\right)}^{(m) *} \ldots \psi_{-\frac{1}{2}}^{(m) *}\right) \\
& \times\left(\varphi_{-\frac{1}{2}}^{(1)}\right)^{c_{1}} \ldots\left(\varphi_{-\frac{1}{2}}^{(n)}\right)^{c_{n}}\left(\varphi_{-\frac{1}{2}}^{(1) *}\right)^{d_{1}} \ldots\left(\varphi_{-\frac{1}{2}}^{(n) *}\right)^{d_{n}}|0\rangle .
\end{aligned}
$$


All other basis elements from $F(a, b, c, d)$ are obtained from $v(a, b, c, d)$ by adding to the lower indices of the factors arbitrary non-negative integers. Hence we have (since weight $|0\rangle=\Lambda_{0}$ ):

$$
\begin{aligned}
\operatorname{ch} F(a, b, c, d) & =e^{\text {weight }(v(a, b, c, d))} / \Pi(q), \text { where } \\
\Pi(q) & =(q)_{a_{1}} \ldots(q)_{a_{m}}(q)_{b_{1}} \ldots(q)_{b_{m}}(q)_{c_{1}} \ldots(q)_{c_{n}}(q)_{d_{1}} \ldots(q)_{d_{n}} .
\end{aligned}
$$

Here and further we use the usual notation and assumptions:

$$
(q)_{a}=(1-q) \ldots\left(1-q^{a}\right), q=e^{-\delta} \text { and }|q|<1 \text {. }
$$

Noticing that

$$
\begin{aligned}
& \operatorname{weight}\left(\psi_{k}^{(i)}\right)=\epsilon_{i}+k \delta, \text { weight }\left(\psi_{k}^{(i) *}\right)=-\epsilon_{i}+k \delta, \\
& \operatorname{weight}\left(\varphi_{k}^{(i)}\right)=\epsilon_{m+i}+k \delta, \text { weight }\left(\varphi_{k}^{(i) *}\right)=-\epsilon_{m+i}+k \delta,
\end{aligned}
$$

we obtain from (3.11) and (3.12) the "quasiparticle" character formula for $F_{s}$ :

$$
\operatorname{ch} F_{s}=e^{\Lambda_{0}} \sum_{\substack{a, b \in \mathbb{Z}_{+}^{m+n} \\|a|-|b|=s}} \frac{e^{\sum_{i=1}^{m+n}\left(a_{i}-b_{i}\right) \epsilon_{i}} q^{\frac{1}{2} \sum_{i=1}^{m}\left(a_{i}^{2}+b_{i}^{2}\right)+\frac{1}{2} \sum_{i=m+1}^{m+n}\left(a_{i}+b_{i}\right)}}{\Pi_{i=1}^{m+n}(q)_{a_{i}}(q)_{b_{i}}} .
$$

Another formula, which we call a theta function type character formula, is derived as follows. Let

$$
\operatorname{ch} F=\sum_{s \in \mathbb{Z}} z^{s} \operatorname{ch} F_{s}
$$

Using (3.3) and (3.13), we obtain:

$$
\operatorname{ch} F=e^{\Lambda_{0}} \Pi_{k=1}^{\infty} \frac{\Pi_{i=1}^{m}\left(1+z e^{\epsilon_{i}} q^{k-1 / 2}\right)\left(1+z^{-1} e^{-\epsilon_{i}} q^{k-1 / 2}\right)}{\Pi_{j=1}^{n}\left(1-z e^{\epsilon_{m+j}} q^{k-1 / 2}\right)\left(1-z^{-1} e^{-\epsilon_{m+j}} q^{k-1 / 2}\right)} .
$$

In order to compute the coefficient of $z^{s}$, we use the Jacobi triple product identity

$$
\Pi_{k=1}^{\infty}\left(1+z q^{k-\frac{1}{2}}\right)\left(1+z^{-1} q^{k-\frac{1}{2}}\right)=\frac{1}{\varphi(q)} \sum_{m \in \mathbb{Z}} z^{m} q^{\frac{1}{2} m^{2}},
$$

and also the following well-known identity which can be derived from the super boson-fermion correspondence [K4]:

$$
\begin{array}{r}
\Pi_{k=1}^{\infty}\left(1+z q^{k-\frac{1}{2}}\right)^{-1}\left(1+z^{-1} q^{k-\frac{1}{2}}\right)^{-1}=\frac{1}{\varphi(q)^{2}} \sum_{m \in \mathbb{Z}}(-1)^{m} \frac{q^{\frac{1}{2} m(m+1)}}{1+z q^{m+\frac{1}{2}}} \\
=\varphi(q)^{-2}\left(\sum_{m, k \geq 0}-\sum_{m, k<0}\right)\left((-1)^{m+k} z^{k} q^{\frac{1}{2} m(m+1)+\left(m+\frac{1}{2}\right) k}\right) .
\end{array}
$$

Here and further 


$$
\varphi(q)=\Pi_{j=1}^{\infty}\left(1-q^{j}\right) .
$$

Substituting (3.16) and (3.17) in (3.15), we get:

$$
\begin{aligned}
\operatorname{ch} F= & \frac{e^{\Lambda_{0}}}{\varphi(q)^{m+2 n}} \sum_{k \in \mathbb{Z}^{m}}\left(\sum_{p_{1}, a_{1} \geq 0}-\sum_{p_{1}, a_{1}<0}\right) \ldots\left(\sum_{p_{n}, a_{n} \geq 0}-\sum_{p_{n}, a_{n}<0}\right) \\
& (-1)^{|r|} z^{|k|+|p|} e^{\sum_{i} k_{i} \epsilon_{i}+\sum_{j} p_{j} \epsilon_{m+j}} q^{\frac{1}{2} \sum_{i} k_{i}^{2}+\frac{1}{2} \sum_{j}\left(a_{j}\left(a_{j}+1\right)+p_{j}\left(a_{j}+1 / 2\right)\right)},
\end{aligned}
$$

where $k=\left(k_{1}, \ldots, k_{m}\right) \in \mathbb{Z}^{m}, p=\left(p_{1}, \ldots, p_{n}\right), a=\left(a_{1}, \ldots, a_{n}\right) \in \mathbb{Z}^{n}$, and $|k|=\sum_{i} k_{i}$.

The coefficient of $z^{s}$ is a rather complicated expression for $c h F_{s}$, which, after letting $r=$ $p+a \in \mathbb{Z}^{n}$, can be written as follows:

$$
c h F_{s}=\frac{e^{\Lambda_{0}+s \epsilon_{1}} q^{s^{2} / 2}}{\varphi(q)^{m+2 n}} \sum_{k \in \mathbb{Z}^{m-1}}\left(\sum_{r_{1} \geq p_{1} \geq 0}-\sum_{r_{1}<p_{1}<0}\right) \ldots\left(\sum_{r_{n} \geq p_{n} \geq 0}-\sum_{r_{n}<p_{n}<0}\right)
$$

$$
(-1)^{|r+p|} e^{\sum_{i} k_{i}\left(\epsilon_{i}-\epsilon_{1}\right)+\sum_{j} p_{j}\left(\epsilon_{m+j}-\epsilon_{1}\right)} q^{\frac{1}{2}|k|^{2}+\frac{1}{2} \sum_{i} k_{i}^{2}+\frac{1}{2} \sum_{j} r_{j}\left(r_{j}+1\right)+\sum_{i<j} p_{i} p_{j}+|k||p|-s(|k|+|p|)},
$$

where $k=\left(k_{2}, \ldots, k_{m}\right) \in \mathbb{Z}^{m-1}, p, r \in \mathbb{Z}^{n}$.

We rewrite (3.18) using translation operators $t_{\alpha}, \alpha \in \mathfrak{h}^{*}$, defined by $\left(\lambda \in \widehat{\mathfrak{h}}^{*}\right)$ :

$$
t_{\alpha}(\lambda)=\lambda+(\lambda \mid \delta) \alpha-\left(\frac{1}{2}(\alpha \mid \alpha)(\lambda \mid \delta)+(\lambda \mid \alpha)\right) \delta .
$$

Let $M^{\#}=\sum_{i=1}^{m-1} \mathbb{Z} \alpha_{i}$; recall that $M^{\#}$ acts on $\widehat{\mathfrak{h}}^{*}$ via $\alpha \mapsto t_{\alpha}$ and the image of this action is the translation subgroup of the Weyl group of $s \ell(m)$. It is straightforward to show that (3.18) can be rewritten as follows:

$$
\begin{gathered}
c h F_{s}=\frac{1}{\varphi(q)^{m+2 n}}\left(\sum_{r_{1} \geq p_{1} \geq 0}-\sum_{r_{1}<p_{1}<0}\right) \cdots\left(\sum_{r_{n} \geq p_{n} \geq 0}-\sum_{r_{n}<p_{n}<0}\right) \\
(-1)^{|r|+|p|} q^{\frac{1}{2} \sum_{j} r_{j}\left(r_{j}+1\right)+\sum_{i<j} p_{i} p_{j}-|p|+a_{s}} \sum_{\alpha \in M^{\#}} e^{t_{\alpha}\left(\Lambda_{(s)}-\sum_{j=1}^{n} p_{j}\left(\epsilon_{1}-\epsilon_{m+j}\right)\right)}
\end{gathered}
$$

where $\Lambda_{(s)}$ is the weight of $|s\rangle$ and $a_{s}=s\left(r_{n}-p_{n}+1\right)+|p|$ if $s \leq 0,=0$ if $0<s \leq m$, and $=(s-m)\left(r_{1}-p_{1}\right)$ if $s \geq m$.

In the case $n=1$ formula (3.18) can be simplified by making use of the following lemma.

Lemma 3.1. Let $a, b \in \mathbb{Z}$. Then $(j, k, n \in \mathbb{Z})$ :

(a) $\left(\sum_{k \geq j \geq a}-\sum_{k<j<a}\right)(-1)^{j+k} x^{j} q^{b k} q^{k(k+1) / 2}=\frac{x}{1+x} \varphi(q) \Pi_{n=1}^{\infty}\left(1+x^{-1} q^{n-b-1}\right)\left(1+x q^{n+b}\right)$.

(b) $\Pi_{n=1}^{\infty}\left(1+x^{-1} q^{n-b-1}\right)\left(1+x q^{n+b}\right)=x^{-b} q^{-b(b+1) / 2} \Pi_{n=1}^{\infty}\left(1+x^{-1} q^{n-1}\right)\left(1+x q^{n}\right)$ $=x^{-b-1} q^{-b(b+1) / 2} \Pi_{n=1}^{\infty}\left(1+x^{-1} q^{n}\right)\left(1+x q^{n-1}\right)$. 
Proof. If $k \geq a$ (resp. $k<a)$, we have:

$$
\sum_{j=a}^{k}(-1)^{j+k} x^{j}\left(\text { resp. }-\sum_{j=k+1}^{a-1}(-1)^{j+k} x^{j}\right)=\frac{x^{k+1}-(-1)^{k} x^{a}}{1+x} .
$$

Hence the LHS of (a) is equal to

$$
\frac{1}{1+x} \sum_{k \in \mathbb{Z}} x^{k+1} q^{b k+k(k+1) / 2}-\frac{x^{a}}{1+x} \sum_{k \in \mathbb{Z}}(-1)^{k} q^{b k+k(k+1) / 2} .
$$

Noticing that the second summand is zero and applying to the first summand the Jacobi triple product identity (3.16), we obtain (a). In the proof of (b) we assume that $b>0$, the case $b<0$ being similar:

$$
\Pi_{n=1}^{\infty}\left(1+x^{-1} q^{n-b-1}\right)=\Pi_{m \geq 1-b}\left(1+x^{-1} q^{m-1}\right)=\Pi_{m=1}^{\infty}\left(1+x^{-1} q^{m-1}\right) \Pi_{m=1-b}^{0}\left(1+x^{-1} q^{m-1}\right) .
$$

The second product on the RHS is equal to

$$
\Pi_{n=1}^{b}\left(1+x^{-1} q^{-n}\right)=\Pi_{n=1}^{b} x^{-1} q^{-n}\left(1+x q^{n}\right)=x^{-b} q^{-b(b+1) / 2} \Pi_{n=1}^{b}\left(1+x q^{n}\right) .
$$

Next, we have:

$$
\Pi_{n=1}^{\infty}\left(1+x q^{n+b}\right)=\Pi_{m=1+b}^{\infty}\left(1+x q^{m}\right)=\Pi_{m=1}^{\infty}\left(1+x q^{m}\right) / \Pi_{m=1}^{b}\left(1+x q^{m}\right) .
$$

These equalities prove (b).

Let now $n=1$. Then 3.18 reads:

$$
c h F_{s}=\frac{e^{\Lambda_{0}+s \epsilon_{1}} q^{s^{2} / 2}}{\varphi(q)^{m+2}} \sum_{k \in \mathbb{Z}^{m-1}} \psi(k) e^{\sum_{i} k_{i}\left(\epsilon_{i}-\epsilon_{1}\right)} q^{\frac{1}{2}|k|(|k|-2 s)+\frac{1}{2} \sum_{i} k_{i}^{2}},
$$

where

$$
\psi(k)=\left(\sum_{t \geq j \geq 0}-\sum_{t<j<0}\right)(-1)^{j+t}\left(q^{|k|-s} e^{\epsilon_{m+1}-\epsilon_{1}}\right)^{j} q^{t(t+1) / 2} .
$$

By Lemma 3.1 a for $x=q^{|k|-s} e^{\epsilon_{m+1}-\epsilon_{1}}, b=0$, we have:

$$
\psi(k)=\frac{q^{|k|-s} e^{\epsilon_{m+1}-\epsilon_{1}} \varphi(q)}{1+q^{|k|-s} e^{\epsilon_{m+1}-\epsilon_{1}}} \Pi_{n=1}^{\infty}\left(1+e^{\epsilon_{1}-\epsilon_{m+1}} q^{n-|k|+s-1}\right)\left(1+e^{\epsilon_{m+1}-\epsilon_{1}} q^{n+|k|-s}\right) .
$$

By Lemma 3.1] for $x=e^{\epsilon_{m+1}-\epsilon_{1}}, b=|k|-s$, we rewrite this as follows:

$$
\psi(k)=\frac{x^{-b} q^{-b(b-1) / 2} \varphi(q)}{1+q^{b} x} \Pi_{n=1}^{\infty}\left(1+x^{-1} q^{n}\right)\left(1+x q^{n-1}\right) .
$$


Substituting this in (3.21), we obtain:

$$
\begin{aligned}
c h F_{s} & =\frac{e^{\Lambda_{0}+s \epsilon_{m+1}} q^{-\frac{s}{2}}}{\varphi(q)^{m+1}} \Pi_{n=1}^{\infty}\left(1+e^{\epsilon_{1}-\epsilon_{m+1}} q^{n}\right)\left(1+e^{\epsilon_{m+1}-\epsilon_{1}} q^{n-1}\right) \\
& \times \sum_{k \in \mathbb{Z}^{m-1}} \frac{e^{\sum_{i=2}^{m} k_{i}\left(\epsilon_{i}-\epsilon_{m+1}\right)}}{1+q^{|k|-s} e^{\epsilon_{m+1}-\epsilon_{1}}} q^{\frac{1}{2} \sum_{i=2}^{m} k_{i}\left(k_{i}+1\right)} .
\end{aligned}
$$

This formula agrees with the one obtained in [KU] (see also [K4]) for $g \ell(1 \mid 1)$.

In the next section we use this formula in the case $m \geq 2$ in order to derive character formulas for all integrable level $1 \mathrm{~s} \ell(\mathrm{m} \mid \mathrm{1})$-modules in terms of the theta function and the (multivariable) Appell's functions, and to obtain their high temperature asymptotics.

\section{Theta function type character formula for integrable level $1 s \ell(m \mid 1)$-modules and Appell's function.}

Recall that Appell's function is defined by the following series (cf. [A] and [P]):

$$
A(x, z, q)=\sum_{k \in \mathbb{Z}} \frac{q^{\frac{1}{2} k^{2}} z^{k}}{1+x q^{k}},
$$

which converges to a meromorphic function in the domain $x, z, q \in \mathbb{C},|q|<1$. The classical theta function in one variable is a special case of this function:

$$
\Theta(z) \equiv \Theta(z ; q)=A(0, z, q) .
$$

Note that by 3.16 we have a product expansion:

$$
\Theta\left(z q^{1 / 2} ; q\right)=\varphi(q) \Pi_{k=1}^{\infty}\left(1+z q^{k}\right)\left(1+z^{-1} q^{k-1}\right) .
$$

We shall need also the following multivariable generalization of Appell's function. Let $B$ be an $N \times N$ symmetric matrix such that $\operatorname{Re} B$ is positive definite and let $\ell$ be a linear function of $\mathbb{C}^{N}$. We define the series

$$
A_{B, \ell}\left(x ; z_{1}, \ldots, z_{N} ; q\right)=\sum_{k \in \mathbb{Z}^{N}} \frac{q^{\frac{1}{2} k^{T} B k} z_{1}^{k_{1}} \ldots z_{N}^{k_{N}}}{1+x q^{\ell(k)}},
$$

which converges to a meromorphic function provided that $|q|<1$. Again, letting $x=0$, we get the multivariable theta function.

Consider now the $g \ell(m \mid 1)$-modules $F_{s}, s \in \mathbb{Z}$, and assume in this section that $m \geq 2$. We have (see Remark 3.2):

$$
\Lambda_{(s)}=\left\{\begin{array}{ll}
\Lambda_{s}-\frac{s}{2} \delta & \text { if } 0 \leq s \leq m \\
-(s-m) \Lambda_{0}+(1+s-m) \Lambda_{m}-\frac{s}{2} \delta & \text { if } s \geq m \\
(1-s) \Lambda_{0}+s \Lambda_{m}+\frac{s}{2} \delta & \text { if } s \leq 0
\end{array} .\right.
$$

For $m \geq 2$, we have: $g \ell(m \mid 1) \hat{)}=s \ell(m \mid 1 \hat{)}+g \ell(1)$ (sum of ideals), hence:

$$
\operatorname{ch} F_{s}=\operatorname{chL}\left(\Lambda_{(s)}\right) \varphi(q)^{-1},
$$


where $L\left(\Lambda_{(s)}\right)$ denotes the irreducible $s \ell(m \mid 1)$-module with highest weight $\Lambda_{(s)}$. Hence formula (3.22) gives us the following expression for $\operatorname{ch} L\left(\Lambda_{(s)}\right)$ in terms of the theta function $\Theta(z ; q)$ (we use (4.1)) and the (multivariable) Appell's function:

$$
\operatorname{chL}\left(\Lambda_{(s)}\right)=\frac{e^{\Lambda_{0}+s \epsilon_{m+1}} q^{-\frac{s}{2}}}{\varphi(q)^{m+1}} \Theta\left(z_{1} q^{\frac{1}{2}} ; q\right) A_{I, \ell}\left(z_{1}^{-1} q^{-s} ; z_{2} q^{\frac{1}{2}}, \ldots, z_{m} q^{\frac{1}{2}} ; q\right),
$$

where $z_{i}=e^{\epsilon_{i}-\epsilon_{m+1}}(i=1, \ldots, m), B=I$ is the $(m-1) \times(m-1)$ identity matrix and $\ell(k)=\sum_{i} k_{i}, k \in \mathbb{Z}^{m-1}$. (Note that in the simplest case $m=2$ we get in this expression the classical Appell's function.)

Next, we derive yet another character formula for the $s \ell(m \mid 1)$-module $L\left(\Lambda_{0}\right)$ in the case $m \geq 2$, in terms of classical theta functions and certain "half" modular forms. We use for this (3.20) for $n=1$ :

$$
\operatorname{ch} L\left(\Lambda_{0}\right)=\frac{1}{\varphi(q)^{m+1}}\left(\sum_{r \geq p \geq 0}-\sum_{r<p<0}\right) \sum_{\alpha \in M^{\#}}(-1)^{r+p} e^{t_{\alpha}\left(\Lambda_{0}-p\left(\epsilon_{1}-\epsilon_{m+1}\right)\right)} q^{\frac{1}{2} r(r+1)} .
$$

Introduce the following elements of $M^{\#}$ :

$$
\beta_{k}=k \epsilon_{1}-\sum_{i=1}^{k} \epsilon_{1}, \quad k=1, \ldots, m
$$

and the element $\mu=\epsilon_{m+1}-\frac{1}{m}\left(\epsilon_{1}+\cdots+\epsilon_{m}\right) \in \mathfrak{h}^{*}$, which is orthogonal to $M^{\#}$. The even part

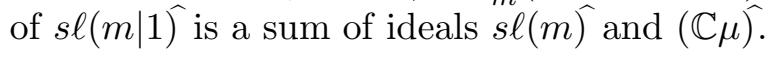

Write $p=j m-k$, where $j \in \mathbb{Z}, 1 \leq k \leq m$ and note that

$$
\Lambda_{0}-p\left(\epsilon_{1}-\epsilon_{m+1}\right)+j \beta_{m}-\beta_{k}=\dot{\Lambda}_{k}+p \mu
$$

where $\dot{\Lambda}_{k}$ denote fundamental weights of $s \ell(m)$ and we identify $\dot{\Lambda}_{m}$ with $\dot{\Lambda}_{0}$. Adding to $\alpha$ the element $j \beta_{m}-\beta_{k}$ in (4.3), and using (4.4), we rewrite (4.3) as follows:

$$
\begin{aligned}
\operatorname{ch} L\left(\Lambda_{0}\right)= & \frac{1}{\varphi(q)^{m+1}} \sum_{k=1}^{m}\left(\sum_{\substack{j, r \in \mathbb{Z} \\
r+k \geq j m \\
j>0}}-\sum_{\substack{j, r \in Z \\
r+k<j m \\
j \leq 0}}\right) \\
& (-1)^{r+k+j m} \sum_{\alpha \in M^{\#}} e^{t_{\alpha}\left(\dot{\Lambda}_{k}\right)+(j m-k) \mu} q^{\frac{r(r+1)}{2}-\frac{(j m-k)(j m-k-j)}{2}-\frac{k(j-1)}{2}} .
\end{aligned}
$$

Denoting by $\dot{L}(\dot{\Lambda}, a)$ the irreducible $s \ell(m)^{\wedge}+(\mathbb{C} \mu)^{\wedge}$-module with highest weight $\dot{\Lambda}+a \mu$, and recalling that ([K3], Proposition 12.13):

$$
\operatorname{ch} \dot{L}\left(\dot{\Lambda}_{k}, a\right)=\frac{1}{\varphi(q)^{m}} \sum_{\alpha \in M^{\#}} e^{t_{\alpha}\left(\dot{\Lambda}_{k}\right)+a \mu},
$$


we obtain:

$$
\operatorname{ch} L\left(\Lambda_{0}\right)=\sum_{k=1}^{m} \sum_{\substack{p \in \mathbb{Z} \\ p+k \mid m}} b_{k, p}(q) \operatorname{ch} \dot{L}\left(\dot{\Lambda}_{k}, p\right)
$$

where

$$
b_{k, p}(q)=\frac{q^{\left(\frac{1}{2}-\frac{1}{2 m}\right) p^{2}+\frac{1}{2 m} k^{2}+\frac{1}{2} k}}{\varphi(q)} \times \sum_{r \geq p}(-1)^{r-p} q^{\frac{1}{2} r(r+1)}\left(\text { resp. } \quad \times \sum_{r<p}(-1)^{r-p-1} q^{\frac{1}{2} r(r+1)}\right),
$$

if $p \geq 0$ (resp. $p<0$ ).

Thus, the branching functions $b_{k, p}(q)$ are "half" modular functions, in a sharp contrast with the case of affine Lie algebras [K3]. Recalling that the series $\sum_{\alpha \in M_{\#}} e^{t_{\alpha}(\Lambda)}$ converges to a classical theta function [K3], we see that the character of the "basic" $s \ell(m \mid 1)$-module is a finite linear combination of classical theta functions with coefficients "half" modular functions.

The basic specialization of $(3.22)$ gives the specialized character formulas for $s \ell(m \mid 1)$ modules $L\left(\Lambda_{(s)}\right)$ :

$$
\operatorname{tr}_{L\left(\Lambda_{(s)}\right)} q^{L_{0}}=2 q^{-\frac{s}{2}} \frac{\varphi\left(q^{2}\right)^{2}}{\varphi(q)^{m+2}} \sum_{k \in \mathbb{Z}^{m-1}} \frac{q^{\frac{1}{2} \sum_{i} k_{i}\left(k_{i}+1\right)}}{1+q^{|k|-s}}
$$

where, as before, $|k|=\sum_{i} k_{i}$.In the remainder of this section we discuss asymptotics of (4.7).

Given a positive definite quadratic form $B(x)$ on $\mathbb{R}^{N}$, an affine linear function $\ell(x)$ on $\mathbb{R}^{N}$ and an element $\alpha$ of $\mathbb{R}^{N}$, consider the following series, where $q=e^{2 \pi i \tau}$ :

$$
f_{B, \ell, \alpha}(\tau)=\sum_{\gamma \in \mathbb{Z}^{N}+\alpha} \frac{q^{\frac{1}{2} B(\gamma)}}{1+q^{\ell(\gamma)}}
$$

This series converges on the upper half plane $\operatorname{Im} \tau>0$ to a specialization of the multivariable Appell's function. From the transformation properties of theta series one gets (see e.g. K3]):

$$
f_{B, 0, \alpha}(i \beta)=\frac{1}{2}(\operatorname{det} B)^{-1 / 2} \beta^{-N / 2}+o(\beta) \text { as } \beta \rightarrow 0 \quad(\operatorname{Re} \beta>0) .
$$

In order to get the asymptotics of the functions $f_{B, \ell, \alpha}(\tau)$, let $f_{B, \ell, \alpha}^{ \pm}(\tau)=\frac{1}{2} \sum_{\substack{\gamma \in \mathbb{Z}^{N}+\alpha \\ \pm(\gamma)>0}} q^{\frac{1}{2} B(\gamma)}$.

It is easy to derive from (4.8) by induction on $n$ the following asymptotics:

$$
f_{B, \ell, \alpha}^{ \pm}(i \beta)=\frac{1}{4}(\operatorname{det} B)^{-1 / 2} \beta^{-N / 2}+p_{ \pm}\left(\beta^{-1 / 2}\right)+o(\beta)
$$

as $\beta \rightarrow 0, \beta \in \mathbb{R}_{+}$, where $p_{ \pm}(x)$ is a polynomial in $x$ of degree strictly less than $N$.

The idea of the following lemma is due to A. Polishchuk.

Lemma 4.1. $\left|f_{B, \ell, \alpha}(i \beta)-f_{B, 0, \alpha}(i \beta)\right|<p\left(\beta^{-1 / 2}\right)$ for $\beta \in \mathbb{R}, 0<\beta<a$, where a is a positive number and $p(x)$ is a polynomial in $x$ of degree strictly less than $N$. 
Proof. Let $g(\beta)=f_{B, \ell, \alpha}(i \beta)-f_{B, 0, \alpha}(i \beta)$. We have: $g(\beta)=g^{+}(\beta)-g^{-}(\beta)$, where

$$
g^{ \pm}(\beta)=\sum_{\substack{\gamma \in \mathbb{Z}^{N}+\alpha \\ \pm \ell(\gamma)>0}} e^{-\pi \beta B(\gamma)} \frac{1-e^{\mp 2 \pi \beta \ell(\gamma)}}{1+e^{\mp 2 \pi \beta \ell(\gamma)}} .
$$

Furthermore, we have:

$$
0 \leq g^{ \pm}(\beta) \leq \sum_{\substack{\gamma \in \mathbb{Z}^{N}+\alpha \\ \pm \ell(\gamma)>0}} e^{-\pi \beta B(\gamma)}-\sum_{\substack{\gamma \in \mathbb{Z}^{N}+\alpha \\ \pm \ell(\gamma)>0}} e^{-\pi \beta(B(\gamma) \pm 2 \ell(\gamma))} .
$$

The first sum on the right is just $f_{B, \ell, \alpha}^{ \pm}(i \beta)$, which has asymptotics (4.9). But the second sum on the right has asymptotics of this form too since it can be written as a product of a power of $q$ and a function $f_{B, \ell^{\prime}, \alpha}^{ \pm}(i \beta)$ for some other affine linear function $\ell^{\prime}$, by "completing the squares". The lemma is proved.

We shall write $f(\tau) \sim g(\tau)$ if $\lim _{\substack{\beta \rightarrow \mathbb{R}_{+} \\ \text {W }}} f(i \beta) / g(i \beta)=1$. Lemma 4.1 and (4.8) imply:

$$
f_{B, \ell, \alpha}(\tau) \sim \frac{1}{2}(\operatorname{det} B)^{-1 / 2} \beta^{-N / 2}
$$

Since

$$
\eta(\tau) \sim \beta^{-1 / 2} e^{-\pi / 12 \beta}
$$

we deduce from (4.10) and (4.7) the following asymptotics along the imaginary axis $\tau=i \beta, \beta \in$ $\mathbb{R}_{+}$:

$$
\operatorname{tr}_{L\left(\Lambda_{(s)}\right)} q^{L_{0}} \sim \frac{1}{2} \beta^{\frac{1}{2}} e^{\frac{\pi}{12 \beta}(m+1)}
$$

\section{$5 \quad$ A Weyl type character formula for integrable level $1 g \ell(m \mid n)$-modules.}

In this section we derive a Weyl type character formula (5.12) for principal integrable level 1modules over $g \ell(m \mid n)$ provided that $m \geq n$. We use for that formula (3.15) for $c h F$ and the denominator identity for $s \ell(m+1 \mid n) \hat{)}$. In order to compare these two formulas, we consider the labelings of simple roots of $g \ell(m \mid n)$ and $s \ell(m+1 \mid n)$ given below:
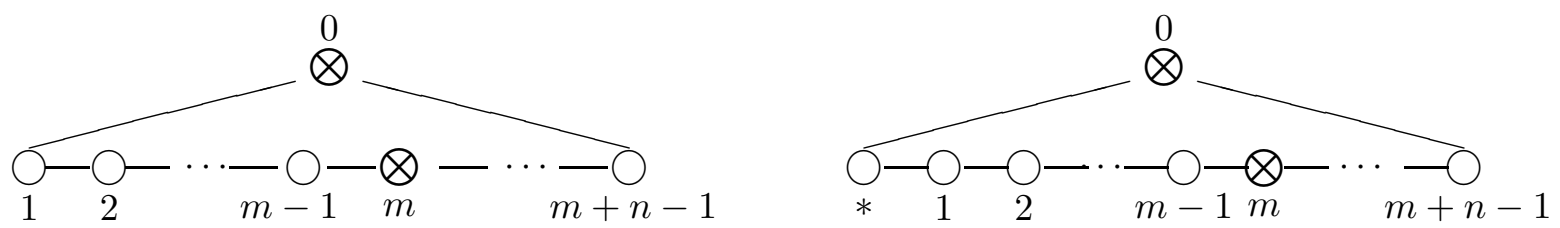

Putting

$$
z=-e^{-\epsilon_{1}} q^{-\frac{1}{2}} y \text { and } y=e^{-\alpha_{*}},
$$


we can rewrite formula (3.15) as follows:

$$
e^{-\Lambda_{0}} \operatorname{ch} F=\Pi_{k=1}^{\infty} \quad \frac{\Pi_{i=0}^{m-1}\left(1-e^{\alpha_{*}+\alpha_{1}+\cdots+\alpha_{i}} q^{k}\right)\left(1-e^{-\alpha_{*}-\alpha_{1}-\cdots-\alpha_{i}} q^{k-1}\right)}{\Pi_{j=0}^{n-1}\left(1+e^{\alpha_{*}+\alpha_{1}+\cdots+\alpha_{m+j}} q^{k}\right)\left(1+e^{-\alpha_{*}-\alpha_{1}-\cdots-\alpha_{m}+j} q^{k-1}\right)} .
$$

Denote by $W^{\#}$ (resp. $\left.\tilde{W}^{\#}\right)$ the subgroup of the Weyl group of $g \ell(m \mid n)$ (resp. $\left.s \ell(m+1 \mid n)\right)$ generated by reflections $r_{\alpha}$ in roots $\alpha=\alpha_{1}, \ldots, \alpha_{m-1}\left(\operatorname{resp} . \alpha_{*}, \alpha_{1}, \ldots, \alpha_{m-1}\right)$ and by $M^{\#}$ (resp. $\tilde{M}^{\#}$ ) the subgroups of these Weyl groups generated by translations $t_{\alpha}$ in integral linear combinations of these roots. Let $\tilde{\rho}$ denote a Weyl vector for $s \ell(m+1 \mid n) \hat{)}$ and let $R$ denote the

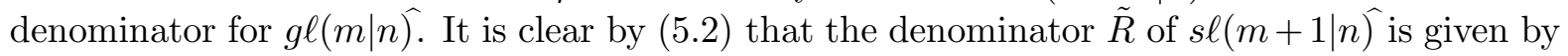

$$
\tilde{R}=e^{-\Lambda_{0}} R \operatorname{ch} F \text {. }
$$

In order to write down the denominator identity for $s \ell\left(m+1 \mid n \hat{)}\right.$, introduce the roots $\beta_{i j}=$ $\alpha_{i}+\alpha_{i+1}+\cdots+\alpha_{j}, 1 \leq i \leq j \leq m+n-2\left(\right.$ here $\left.\beta_{i i}=\alpha_{i}\right)$, and let $\beta_{i}=\beta_{m-n+i, m+i-1}$, $i=1, \ldots, n$. Let

$$
\tilde{\rho}^{\prime}=\tilde{\rho}+\sum_{\substack{m-n+2 \leq i \leq m \leq j \leq m+n-2 \\ j-i \leq n-2}} \beta_{i j}
$$

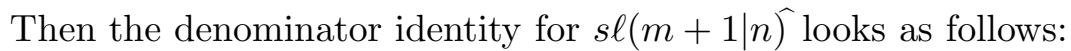

$$
e^{-\Lambda_{0}} e^{\tilde{\rho}^{\prime}} R \operatorname{ch} F=\sum_{w \in \tilde{W}^{\#} \ltimes \tilde{M}^{\#}} \epsilon(w) w \frac{e^{\tilde{\rho}^{\prime}}}{\Pi_{j=1}^{n}\left(1+e^{-\beta_{j}}\right)} .
$$

This identity can be derived from the denominator identity given in $\mathrm{KW}$ by making use of odd reflections as follows.

The denominator identity for $s \ell(m+1 \mid n)$ in $[\overline{K W}]$ is given for the choice of the set of simple roots with a maximal number of grey nodes:

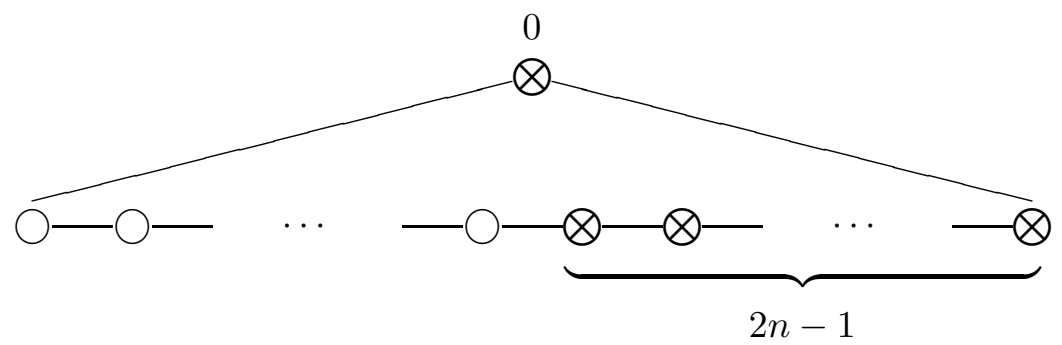

Let $\gamma_{1}, \ldots \gamma_{n}$ be the (unique subset) of the set of simple roots without $\alpha_{0}$ such that $\left(\gamma_{i} \mid \gamma_{j}\right)=0$ for all $i, j$, and let $\tilde{\rho}^{\prime \prime}$ be its Weyl vector. Then the identity reads:

$$
e^{\tilde{\rho}^{\prime \prime}} \tilde{R}=\sum_{w \in \tilde{W}^{\#} \ltimes \tilde{M}^{\#}} \epsilon(w) w \frac{e^{\tilde{\rho}^{\prime \prime}}}{\Pi_{j=1}^{n}\left(1+e^{-\gamma_{j}}\right)} .
$$

In order to derive (5.5) from (5.6), we apply a sequence of odd reflections which transforms the initial diagram with two grey nodes to the above final diagram with $2 n$ grey nodes. In order to 
explain this sequence, denote by $\Pi^{(i, j)}$ a set of simple roots containing $\beta_{i, m+j}$ and by $\Pi^{(i, j+1)}$ the set of simple roots obtained from it by the odd reflection in $\beta_{i, m+j}$. Denoting by $\tilde{\Pi}$ and $\tilde{\Pi}^{\prime}$ the initial and the final sets of simple roots, we have the following sequence:

$$
\begin{aligned}
\tilde{\Pi} & =\Pi^{(m, 0)} \rightarrow \Pi^{(m, 1)} \rightarrow \cdots \rightarrow \Pi^{(m, n-2)} \rightarrow \Pi^{(m, n-1)} \\
& =\Pi^{(m-1,0)} \rightarrow \Pi^{(m-1,1)} \rightarrow \cdots \rightarrow \Pi^{(m-1, n-2)} \\
& =\Pi^{(m-2,0)} \rightarrow \Pi^{(m-2,1)} \rightarrow \cdots \Pi^{(m-2, n-3)} \\
& =\Pi^{(m-3,0)} \rightarrow \Pi^{(m-3,1)} \rightarrow \cdots \\
\cdots & =\Pi^{(m-n+2,0)} \rightarrow \Pi^{(m-n+2,1)}=\tilde{\Pi}^{\prime} .
\end{aligned}
$$

Using Lemma 1.3, one sees that $\tilde{\rho}^{\prime}$ and $\tilde{\rho}$ are related by formula (5.4) and using Lemma 1.4a, we see that

$$
\Lambda_{0}^{\prime}=\Lambda_{0}
$$

Using the decompositions

$$
\tilde{W}^{\#}=W^{\#} \sqcup\left(\sqcup_{j=0}^{m-1} W^{\#} r_{\alpha_{*}+\alpha_{1}+\cdots+\alpha_{j}}\right) \text { and } \tilde{M}^{\#}=\mathbb{Z} \alpha_{*}+M^{\#},
$$

we obtain from $(5.5)$ :

$$
e^{-\Lambda_{0}} R \operatorname{chF}=I_{0}-\sum_{i=0}^{m-1} I I_{i},
$$

where

$$
\begin{aligned}
I_{0} & =e^{-\tilde{\rho}^{\prime}} \sum_{k \in \mathbb{Z}} \sum_{\alpha \in M^{\#}} \sum_{w \in W^{\#}} \epsilon(w) t_{k \alpha_{*}} t_{\alpha} w \frac{e^{\tilde{\rho}^{\prime}}}{\Pi_{j=1}^{n}\left(1+e^{-\beta_{j}}\right)} \\
I_{i} & =e^{-\tilde{\rho}^{\prime}} \sum_{k \in \mathbb{Z}} \sum_{\alpha \in M^{\#}} \sum_{w \in W^{\#}} \epsilon(w) t_{k \alpha_{*}} t_{\alpha} w r_{\alpha_{*}+\alpha_{1}+\cdots+\alpha_{i}} \frac{e^{\tilde{\rho}^{\prime}}}{\Pi_{j=1}^{n}\left(1+e^{-\beta_{j}}\right)} .
\end{aligned}
$$

In order to compute $\operatorname{ch} L\left(\Lambda_{0}\right)$ of the $g \ell(m \mid n)$-module $L\left(\Lambda_{0}\right)$ we compare the constant terms (i.e., $y^{0}$-terms) in the decomposition of both sides of (5.8) is the series in powers of $y$.

We will show that

$$
\begin{aligned}
\text { constant term of } I I_{i} & =0 \text { for all } i \\
\text { constant term of } I_{0} & =\sum_{w \in W^{\#} \ltimes M^{\#}} \epsilon(w) w \frac{e^{\tilde{\rho}^{\prime}}}{\Pi_{j=1}^{n}\left(1+e^{-\beta_{j}}\right)} .
\end{aligned}
$$

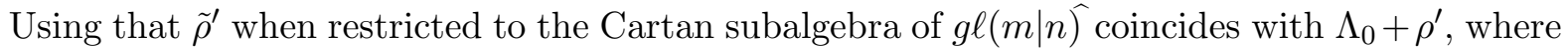
$\rho^{\prime}$ is related to the Weyl vector $\rho$ of $g \ell(m \mid n)$ by (5.4) with $\sim$ removed, we obtain from (5.5), (5.9) and (5.10):

$$
e^{\rho^{\prime}} \operatorname{Rch} L\left(\Lambda_{0}\right)=\sum_{w \in W^{\#} \ltimes M^{\#}} \epsilon(w) w \frac{e^{\Lambda_{0}+\rho^{\prime}}}{\Pi_{j=1}^{n}\left(1+e^{-\beta_{j}}\right)} .
$$


Applying to this formula odd reflections as above, we obtain an equivalent character formula for the choice of the diagram with $2 n$ grey nodes as above (where $\rho$ is a Weyl vector for this choice of the diagram):

$$
e^{\rho} \operatorname{Rch} L\left(\Lambda_{0}\right)=\sum_{w \in W^{\#} \ltimes M^{\#}} \epsilon(w) w \frac{e^{\Lambda_{0}+\rho}}{\Pi_{j=1}^{n}\left(1+e^{-\alpha_{m-n+2 j-1}}\right)} .
$$

The proof of (5.9) and (5.10) is straight-forward, and we explain it in the case $n=1$. We have:

$$
e^{-\tilde{\rho}} t_{-k \alpha_{*}} r_{\alpha_{*}+\alpha_{1}+\cdots+\alpha_{m-1}}\left(\frac{e^{\tilde{\rho}}}{1+e^{-\alpha_{m}}}\right)=\frac{e^{-m k \alpha_{*}} e^{-m\left(\alpha_{*}+\alpha_{1}+\cdots+\alpha_{m-1}\right)} q^{k^{2} m-k+m k}}{1+e^{-\left(\alpha_{*}+\alpha_{1}+\cdots+\alpha_{m}\right)} q^{k}} .
$$

Hence:

$$
e^{-\tilde{\rho}} \sum_{k \in \mathbb{Z}} t_{-k \alpha_{*}} r_{\alpha_{*}+\alpha_{1}+\cdots+\alpha_{m-1}}\left(\frac{e^{\tilde{\rho}}}{1+e^{-\alpha_{m}}}\right)=\left(\sum_{k, s \geq 0}-\sum_{k, s<0}\right)(-1)^{s} y^{m(k+1)+s}(\ldots),
$$

where $(\ldots)$ doesn't involve $y$. But the constant term of the last expression is 0 since $m(k+1)+s>0$ if $k, s \geq 0$ and $m(k+1)+s \leq s<0$ if $k, s<0$. Thus, constant term of $I I_{m-1}=0$. Furthermore, we have for $0 \leq i \leq m-2$ :

$$
e^{-\tilde{\rho}} t_{k \alpha_{*}} r_{\alpha_{*}+\alpha_{1}+\cdots+\alpha_{i}} \frac{e^{\tilde{\rho}}}{1+e^{-\alpha_{m}}}=y^{-m k+i+1}(\ldots) .
$$

Since $-m k+i+1 \neq 0$ if $0 \leq i \leq m-2$, we see that constant term of $I I_{i}$ is 0 for all $i$. Finally:

$$
e^{-\tilde{\rho}} t_{k \alpha_{*}} \frac{e^{\tilde{\rho}}}{1+e^{-\alpha_{m}}}=y^{-m k} \frac{q^{k^{2} m+k}}{1+e^{-\alpha_{m}}},
$$

hence the constant term of this expression is equal $\delta_{k, 0}\left(1+e^{-\alpha_{m}}\right)^{-1}$, which proves $(5.10)$.

Using odd reflections one may derive from (5.11) or (5.12) the Weyl-type character formulas for all other level 1 principal integrable modules. For example, in the case $\widehat{\mathfrak{g}}=s \ell(m \mid 1)$, we let $k=m j+s \in \mathbb{Z}_{+}$, where $j \in \mathbb{Z}_{+}$and $0 \leq s \leq m-1$; then

$$
\begin{aligned}
e^{\rho} \operatorname{Rch}\left((k+1) \Lambda_{0}-k \Lambda_{m}\right) & =\sum_{w \in \widehat{W}} \epsilon(w) w \frac{e^{\Lambda+\rho}}{1+e^{-j \delta-\alpha_{m-s}-\ldots-\alpha_{m}}}, \\
e^{\rho} \operatorname{Rch}\left(-k \Lambda_{0}+(k+1) \Lambda_{m}\right) & =\sum_{w \in \widehat{W}} \epsilon(w) w \frac{e^{\Lambda+\rho}}{1+e^{-j \delta-\alpha_{0}-\ldots-\alpha_{s}}},
\end{aligned}
$$

and for $1 \leq j \leq m-1$ we have:

$$
e^{\rho} \operatorname{Rch}\left(\Lambda_{j}\right)=\sum_{w \in \widehat{W}} \epsilon(w) w \frac{e^{\Lambda_{j}+\rho}}{1+e^{-\alpha_{m}}} .
$$




\section{Classification of integrable highest weight modules over affine superalge- bras.}

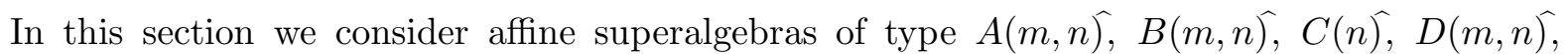
$D(2,1 ; a) \hat{,}, F(4) \hat{)}, G(3)$. We shall exclude from consideration the well understood case of $B(0, n)$ (see [K2] and Section 9.5). In all cases except for $A(n, n)$ these are the affine superalgebras $\widehat{\mathfrak{g}}$ defined by (0.1), (0.2) for $\mathfrak{g}=A(m, n)(m \neq n), B(m, n), C(n), D(m, n), D(2,1 ; a), F(4)$ and $G(3)$ respectively (see [K1] for a construction of the simple finite-dimensional Lie superalgebras $\mathfrak{g})$. In the $A(n, n)$ case it is more convenient to take $\mathfrak{g}=s \ell(n+1 \mid n+1)$ in order not to lose the most interesting modules. The Lie superalgebra $\mathfrak{g}$ carries a unique, up to a constant factor, non-zero invariant bilinear form (.|.). This form extends to $\widehat{\mathfrak{g}}$ by formula (0.3) and it is normalized by the values of $\left(\alpha_{i} \mid \alpha_{i}\right)$, given in Table 1 (see below).

It is convenient to depict Cartan matrices of affine (super)algebras by (generalized) Dynkin diagrams (cf. [K1]). We shall assume that the diagonal entries of a Cartan matrix $\widehat{A}$ are always 2 or 0 (one can achieve this by rescaling simple coroots). The Dynkin diagram of $\widehat{A}$ is a graph whose nodes label the index set $\widehat{I}=\{0,1,2, \ldots\}$ and are of the form $0, \otimes$ or $\boldsymbol{O}$ corresponding to cases $a_{i i}=2, i \notin \widehat{I}_{1} ; a_{i i}=0$ (then $i \in \widehat{I}_{1}$ ); and $a_{i i}=2, i \in \widehat{I}_{1}$, respectively. These nodes are called white, grey and black respectively, so that $\widehat{I}_{1}$ consists of non-white nodes. We let $I=\widehat{I} \backslash\{0\}, I_{1}=\widehat{I}_{1} \backslash\{0\}$. As usual, $I$ labels simple roots $\alpha_{1}, \alpha_{2}, \ldots$ of $\mathfrak{g}, I_{1}$ labels odd simple roots of $\mathfrak{g}$, and $\alpha_{0}=\delta-\theta$, where $\theta$ is the highest root of $\mathfrak{g}$. In the cases $a_{i j}=a_{j i}=0, i \neq j$, the $i^{\text {th }}$ and $j^{\text {th }}$ nodes are not connected. In the cases $a_{i i}=a_{j j}=2, i \neq j$, the nodes are, as usual, connected by $\left|a_{i j} a_{j i}\right|$ edges with an arrow pointing to $j^{\text {th }}$ node if $\left|a_{j i}\right|>1$. In the remaining cases the nodes are joined as follows:

$$
\otimes-\bigcirc=\left(\begin{array}{rr}
0 & a \\
-1 & 2
\end{array}\right), \otimes \Rightarrow \bigcirc=\left(\begin{array}{rr}
0 & a \\
-2 & 2
\end{array}\right)=\otimes \Rightarrow \mathbf{O}, \otimes-\otimes=\left(\begin{array}{ll}
0 & a \\
b & 0
\end{array}\right), \bigcirc \Rightarrow \mathbf{\bigcirc}=\left(\begin{array}{rr}
2 & -1 \\
-2 & 2
\end{array}\right) .
$$

In Table 1 below we list the Dynkin diagrams of the symmetrizable Cartan matrices of the affine Lie superalgebras $\widehat{\mathfrak{g}}$ under consideration. The labels against the nodes $i$ are $\left(\alpha_{i} \mid \alpha_{i}\right)$, and the labels against the edges connecting $i$ and $j$ are $\left(\alpha_{i} \mid \alpha_{j}\right)$. Recall that $\alpha_{i}^{\vee}=2 \alpha_{i} /\left(\alpha_{i} \mid \alpha_{i}\right)$ if $a_{i i} \neq 0$; we let $\alpha_{i}^{\vee}=\alpha_{i}$ if $a_{i i}=0$. We also give the coefficients of the decomposition of the root $\delta$ in terms of simple roots. The nodes are numbered by $\widehat{I}=\{0,1, \ldots\}$ in increasing order from left to right, except when it is impossible to do, in which case nodes are numbered by the subscripts of their labels.

Remark 6.1. Recall the definition of the orthosymplectic Lie subalgebra $\operatorname{osp}(M \mid N)$ [K1]. Let $V=V_{\overline{1}} \oplus V_{\overline{0}}$ be a superspace, where $\operatorname{dim} V_{\overline{1}}=N, \operatorname{dim} V_{\overline{0}}=M$, and let (.|.) be a non-degenerate bilinear form on $V$ such that $\left(V_{\overline{0}} \mid V_{\overline{1}}\right)=0$, the restriction of (.|.) to $V_{\overline{1}}$ is symmetric and to $V_{\overline{0}}$ is skewsymmetric, so that $N=2 n$ is even; let $m=[M / 2]$. Then $(\alpha=0,1)$ :

$$
o s p(M \mid N)_{\bar{\alpha}}=\left\{a \in g \ell(M \mid N)_{\bar{\alpha}} \mid(a(x) \mid y)+(-1)^{\alpha p(x)}(x \mid a(y))=0, \quad x, y \in V\right\} .
$$

For the definition in a matrix form, consider the following $(M+N) \times(M+N)$ matrices:

$$
C=\left(\begin{array}{ll}
C_{1} & 0 \\
0 & C_{2}
\end{array}\right), \quad F=\left(\begin{array}{ll}
I_{M} & 0 \\
0 & -I_{N}
\end{array}\right)
$$


Table 1:

$\widehat{\mathfrak{g}} \quad$ Dynkin diagram

$\delta$

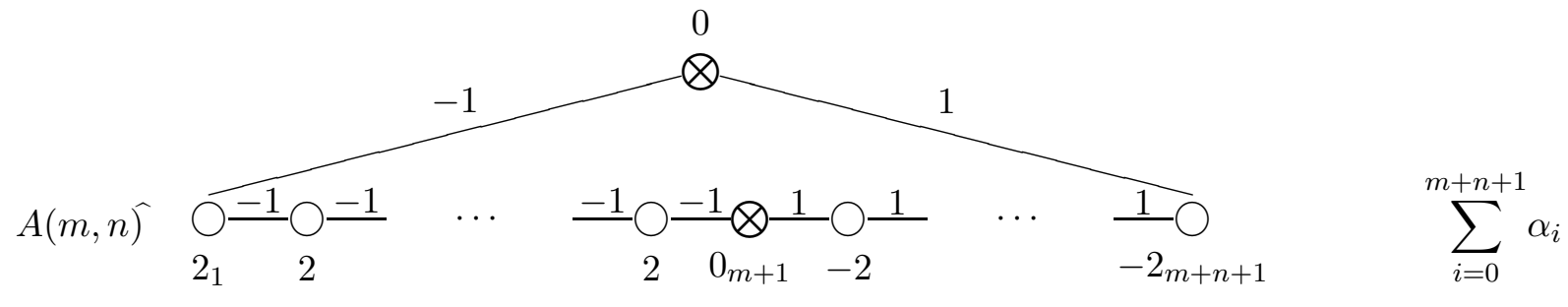

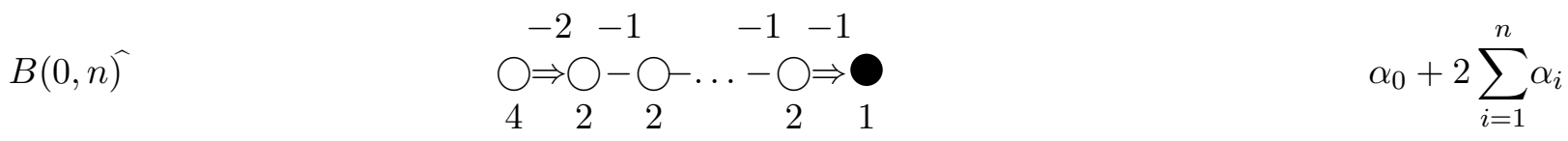

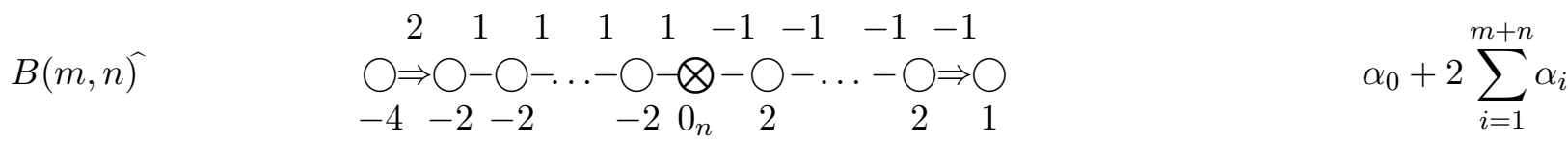

$m>0$

$\bigotimes$

$-2 \bigwedge 1$

$C(n)$

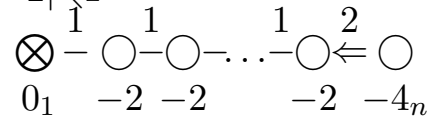

$D(m, n)$

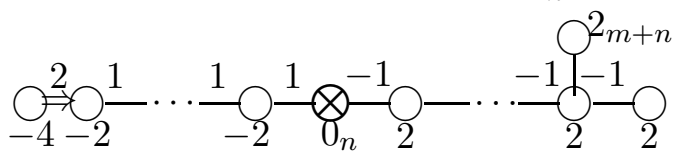

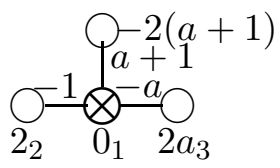

$F(4)$

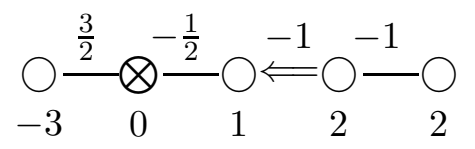

$G(3)$ $\alpha_{0}+2 \alpha_{1}+\alpha_{2}+\alpha_{3}$

$\alpha_{0}+\alpha_{1}+2 \sum_{i=2}^{n-1} \alpha_{i}+\alpha_{n}$

$\alpha_{0}+2 \sum_{i=1}^{m+n-2} \alpha_{i}+\alpha_{m+n-1}+\alpha_{m+n}$

$\alpha_{0}+2 \alpha_{1}+3 \alpha_{2}+2 \alpha_{3}+\alpha_{4}$

$$
\alpha_{0}+2 \alpha_{1}+4 \alpha_{2}+2 \alpha_{3}
$$


where $C_{1}\left(\right.$ resp. $\left.C_{2}\right)$ is a $M \times M($ resp. $N \times N)$ symmetric (resp. skewsymmetric) matrix. Then

$$
o s p(M \mid N)_{\bar{\alpha}}=\left\{a \in g \ell(M \mid N)_{\bar{\alpha}} \mid F^{\alpha} a^{\top} C+C a=0\right\}, \quad \alpha=0,1 .
$$

Recall that $B(m, n)=\operatorname{osp}(2 m+1 \mid 2 n), C(n)=\operatorname{osp}(2 \mid 2 n)$ and $D(m, n)=\operatorname{osp}(2 m \mid 2 n)$. The invariant bilinear form on $\operatorname{osp}(M \mid N)$ that is used in Table 1 and throughout the paper is

$$
(a \mid b)=\frac{1}{2} \operatorname{str} a b .
$$

Table 2:

\begin{tabular}{cc|cc}
\hline \hline $\mathfrak{g}$ & $\mathfrak{g}_{\overline{0}}$ & $\mathfrak{g}$ & $\mathfrak{g}_{\overline{0}}$ \\
\hline$A(m, n)$ & $A_{m}+A_{n}+\mathbb{C}$ & $D(2,1 ; a)$ & $D_{2}+A_{1}$ \\
$C(n)$ & $\mathbb{C}+C_{n}$ & $F(4)$ & $B_{3}+A_{1}$ \\
$B(m, n)$ & $B_{m}+C_{n}$ & $G(3)$ & $G_{2}+A_{1}$ \\
$D(m, n)$ & $D_{m}+C_{n}$ & & \\
\hline
\end{tabular}

The even parts $\mathfrak{g}_{\overline{0}}$ of the Lie superalgebras $\mathfrak{g}$ are listed in Table 2 . In the case of $D(2,1 ; a)$, the subalgebra $D_{2}$ corresponds to $\alpha_{2}$ and $\alpha_{3}$ (see Table 1). We denote by $\mathfrak{g}_{\overline{0}}^{\prime}$ (resp. $\mathfrak{g}_{\overline{0}}^{\prime \prime}$ ) the first (resp. second) non-zero summand of $\mathfrak{g}_{0}$ in the decomposition of Table 2. Note that the invariant bilinear form (.|.) (which can be read off from Table 1) is normalized in such a way that it is positive definite on $\mathfrak{g}_{0}^{\prime}$ and negative definite on $\mathfrak{g}_{\overline{0}}^{\prime \prime}$ (except that for $D(2,1 ; a)$ we should assume that $a \in \mathbb{R}_{>}$), and the maximal square length of a root is 2 , except for the cases $B(1, n)$ when it is 1 and $D(2,1 ; a)$ when it is $\max (2,2 a)$. If the Killing form on $\mathfrak{g}$ is non-degenerate, then the form (.|.) is a positive (resp. negative) multiple of the Killing form, in the cases $\mathfrak{g} \cong s \ell(m \mid n)$ with $m>n, \operatorname{osp}(m \mid n)$ with $m>n+2, F(4)$ and $G(3)($ resp. $s \ell(m \mid n)$ with $m<n$ and $o s p(m \mid n)$ with $m<n+2)$.

An irreducible highest weight module over $\widehat{\mathfrak{g}}$ is called principal (resp. subprincipal) integrable module if it is integrable with respect to $\widehat{\mathfrak{g}_{0}^{\prime}}$ (resp. $\widehat{\mathfrak{g}_{\overline{0}}^{\prime \prime}}$ ) and locally finite with respect to $\mathfrak{g}$ (cf. Definition 0.1). As we shall see, the non-trivial principal (resp. subprincipal) highest weight modules have positive (resp. negative) level, except for the cases $\widehat{\mathfrak{g}}=A(0, n)$ and $C(n)$ (resp. $A(n, 0)$ ). It is easy to see that in these cases the only conditions of integrability are $k_{i} \in \mathbb{Z}_{+}$if $i \in I \backslash I_{1}$; we shall exclude these cases from further considerations.

Let $\theta^{\prime}$ be the highest root of $\mathfrak{g}_{0}^{\prime}$; in cases $D(2, n)$ and $D(2,1 ; a)$, which are the only cases when $\mathfrak{g}_{\overline{0}}^{\prime}$ is not simple, we have: $\mathfrak{g}_{\overline{0}}^{\prime}=A_{1}+A_{1}$, and the highest roots are $\theta_{+}^{\prime}=\alpha_{n+1}$ and $\theta_{-}^{\prime}=\alpha_{n+2}$ (where $n=1$ for $D(2,1 ; a)$ ). The root $\theta^{\prime}\left(\right.$ resp. $\left.\operatorname{root} \theta_{ \pm}^{\prime}\right)$ gives rise to a simple root $\alpha_{0}^{\prime}=\delta-\theta^{\prime}$ (resp. simple roots $\alpha_{0 \pm}^{\prime}=\delta-\theta_{ \pm}^{\prime}$ ) of $\mathfrak{g}_{0}^{\prime}$. The corresponding coroot is $\alpha_{0}^{\prime \vee}=2 \alpha_{0}^{\prime} /\left(\alpha_{0}^{\prime} \mid \alpha_{0}^{\prime}\right)$.

In all cases except for $A(m, n)$ and $C(n)$ there is a (unique) simple root $\alpha^{\prime \prime}$ of $\mathfrak{g}_{0}^{\prime \prime}$ (which is a simple Lie algebra), which is not a simple root of $\mathfrak{g}$ [K1]. As we have seen in $\S 2$, the principal integrability in the case of $A(m, n)$ follows from local nilpotency of the root vector $f^{\prime}$ attached to the root $-\alpha_{0}^{\prime}$. In all other cases one has to check in addition the local nilpotency of the root 
vector $f^{\prime \prime}$ attached to the root $-\alpha^{\prime \prime}$ (in order to ensure the local finiteness with respect to $\mathfrak{g}_{0}^{\prime \prime}$; that with respect to $\mathfrak{g}_{\overline{0}}^{\prime}$ follows automatically from the integrability with respect to $\widehat{\mathfrak{g}_{\overline{0}}^{\prime}}$ ). For that reason, as we have seen in $\$ 1$, it is important to introduce the following numbers, where $\rho$ is a Weyl vector for $\widehat{\mathfrak{g}}$ :

$$
b^{\prime}=-\left\langle\rho, \alpha_{0}^{\prime \vee}\right\rangle, \quad b_{ \pm}^{\prime}=-\left\langle\rho, \alpha_{0^{ \pm}}^{\prime \vee}\right\rangle, \quad b^{\prime \prime}=-\left\langle\rho, \alpha^{\prime \prime \vee}\right\rangle .
$$

The values of the numbers $b^{\prime}$ and $b_{ \pm}^{\prime}$ (resp. $b^{\prime \prime}$ ) are given in Table 3 (resp. 4), the first line for $D(2, \ldots)$ in Table 1 being for $b_{+}^{\prime}$ and the second for $b_{-}^{\prime}$. Table 3 contains also the formula for the partial levels

$$
k^{\prime}=\left\langle\Lambda, \alpha_{0}^{\prime \vee}\right\rangle, k_{ \pm}^{\prime}=\left\langle\Lambda, \alpha_{0 \pm}^{\prime \vee}\right\rangle
$$

and the level $k=\langle\Lambda, K\rangle$ of a weight $\Lambda$ in terms of its labels $k_{i}$ and $k^{\prime}, k_{ \pm}^{\prime}$. Table 4 contains also a formula for

$$
k^{\prime \prime}=\left\langle\Lambda, \alpha^{\prime \prime \vee}\right\rangle
$$

and the level $k$ in terms of the $k_{i}$ and $k^{\prime \prime}$.

Theorem 6.1. For an affine superalgebra from Table 1 (recall that $A(0, n) \wedge, B(0, n)^{\wedge}$ and $C(n)^{\wedge}$ are excluded) the labels $\left\{k_{i}\right\}_{i=\widehat{I}}$ of the highest weight of a principal integrable irreducible highest weight module $L(\Lambda)$ are characterized by the following four series of conditions:

(1) $k_{i} \in \mathbb{Z}_{+}$if $i \in I \backslash I_{1}$,

(2) $k^{\prime}\left(\right.$ resp. $k_{ \pm}^{\prime}$ in case $\left.D(2, \ldots)\right) \in \mathbb{Z}_{+}, k^{\prime \prime} \in \mathbb{Z}_{+}$(see Tables 月 and 团),

(3) if $k^{\prime}$ (resp. one of the $\left.k_{ \pm}^{\prime}\right) \leq b^{\prime}$ (see Table (4), there are the supplementary conditions:

$A(m, n), m \geq 1$ : there exists $s \in \mathbb{Z}_{+}, s \leq k^{\prime}$, such that:

$$
k_{m+1}=k_{m+2}+\cdots+k_{m+1+s}+s, k_{m+s+2}=\cdots=k_{m+s+1+n-k^{\prime}}=0,
$$

$B(m, n) \hat{)}, m \geq 2$, and $D(m, n), m \geq 3$ : one of the four possibilities hold:

(i) there exist $r, s \in \mathbb{Z}_{+}, r<s$, such that

$$
k^{\prime}=r+s, k_{j}=0 \text { for } r+1 \leq j \leq m+n \text { and } j \neq s, k_{s}=1,
$$

(ii) there exist $r, s \in \mathbb{Z}_{+}, r \leq s$, such that

$$
k^{\prime}=r+s, k_{j}=0 \text { for } r+1 \leq j \leq m+n, k_{r} \neq 0,
$$

(iii) there exist $r \in \mathbb{Z}_{+}$such that

$$
k^{\prime}=n+r, k_{j}=0 \text { for } r+1 \leq j \leq n-1, k_{n} \neq 0, k_{n}+k_{n+1}+1=0,
$$

(iv) there exist $r \in \mathbb{Z}_{+}$such that

$$
k^{\prime} \geq n+r, k_{j}=0 \text { for } j \geq r+1, k_{r} \neq 0 .
$$


Table 3:

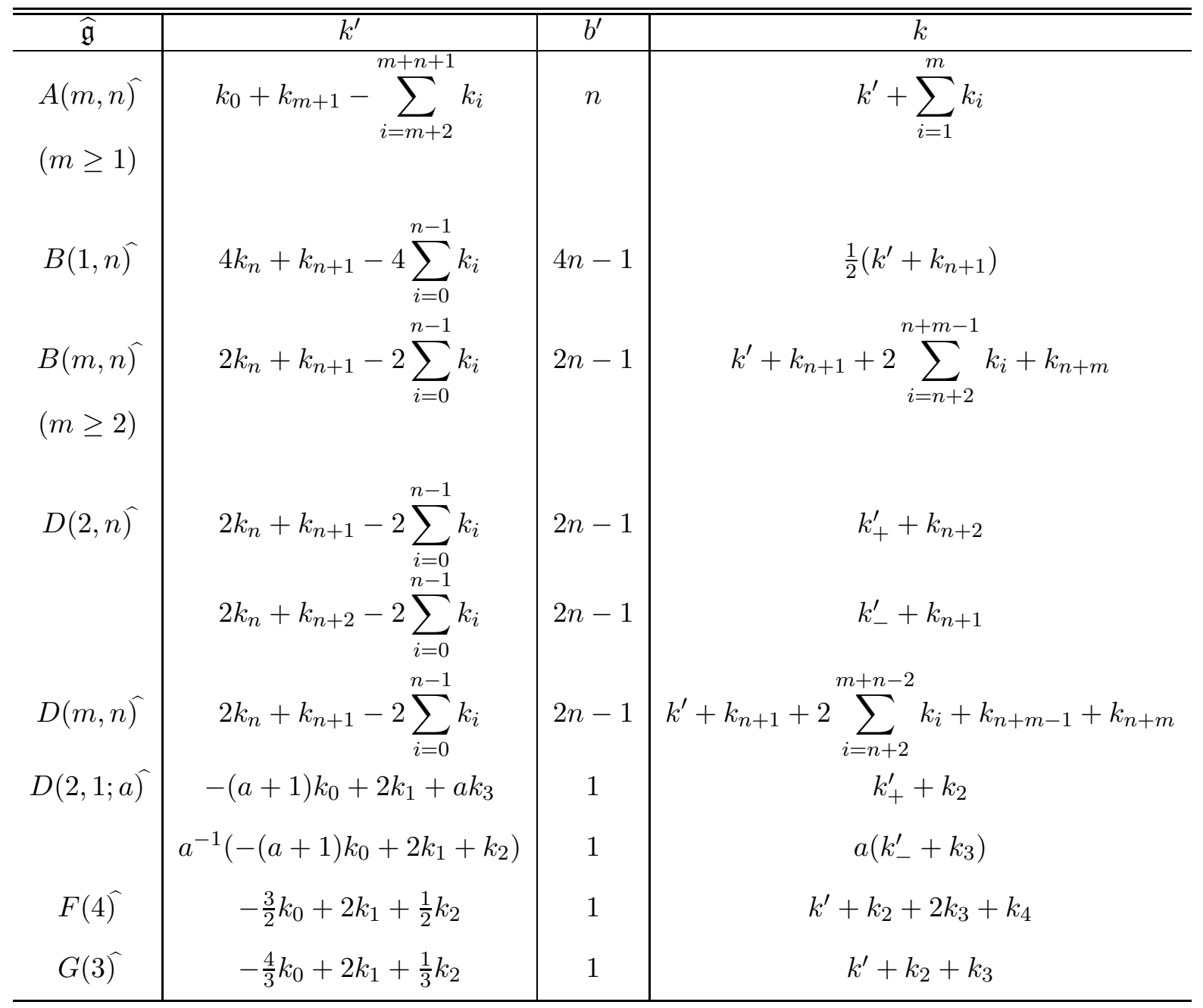


Table 4:

\begin{tabular}{c|c|c|c}
\hline \hline$\hat{\mathfrak{g}}$ & $k^{\prime \prime}$ & $b^{\prime \prime}$ & $\mathrm{k}$ \\
\hline$B(m, n) \hat{)}, m \geq 1$ & $-k_{n}-\sum_{i=n+1}^{n+m-1} k_{i}-\frac{1}{2} k_{m+n}$ & $m-\frac{1}{2}$ & $-2\left(k^{\prime \prime}+\sum_{i=0}^{n-1} k_{i}\right)$ \\
$C(n)$ & $-\frac{1}{2}\left(k_{0}+k_{1}\right)$ & 0 & $-2\left(k^{\prime \prime}+\sum_{i=2}^{n} k_{i}\right)$ \\
$D(m, n) \hat{r}$ & $-k_{n}-\sum_{i=n+1}^{n+m-2} k_{i}-\frac{1}{2}\left(k_{m+n-1}+k_{m+n}\right)$ & $m-1$ & $-2\left(k^{\prime \prime}+\sum_{i=0}^{n-1} k_{i}\right)$ \\
$D(2,1 ; a)$ & $-(a+1)^{-1}\left(2 k_{1}+k_{2}+a k_{3}\right)$ & 1 & $-(a+1)\left(k^{\prime \prime}+k_{0}\right)$ \\
$F(4)$ & $-\frac{4}{3} k_{1}-k_{2}-\frac{4}{3} k_{3}-\frac{2}{3} k_{4}$ & 3 & $-\frac{3}{2}\left(k^{\prime \prime}+k_{0}\right)$ \\
$G(3)$ & $-\frac{3}{2} k_{1}-k_{2}-\frac{3}{2} k_{3}$ & $\frac{5}{2}$ & $-\frac{4}{3}\left(k^{\prime \prime}+k_{0}\right)$ \\
\hline
\end{tabular}

$B(1, n)$ : the same as for $B(m, n)$ with $m>1$ with the following changes: $k^{\prime}$ is replaced by $\frac{1}{2} k^{\prime}$ everywhere and $k_{n+1}$ is replaced by $\frac{1}{2} k_{n+1}$ in (iii),

$D(2, n)$ : the same as for $D(m, n)$ with $m>2$ with the following additions:

$$
k_{+}^{\prime}=k_{-}^{\prime}, k_{n}+k_{n+2}+1=0 \text { in (iii), }
$$

$D(2,1 ; a)$ : one of the four possibilities holds:

(i) $k_{+}^{\prime} k_{-}^{\prime}=0$, then all $k_{i}=0$,

(ii) $a \in \mathbb{Q}_{>0}, a, a^{-1} \notin \mathbb{N}$ and one of $k_{ \pm}^{\prime}$ equals 1 , then $k_{+}^{\prime}=k_{-}^{\prime}=1$

and one has

$\left(^{*}\right) k_{0}=-(a+1)^{-1} r-1, k_{1}=-r, k_{2}=r-1, k_{3}=a^{-1} r-1$ for some $r \in \mathbb{N} \cap a \mathbb{N}$, (iii) $a^{-1} \in \mathbb{N}$ and $k_{+}^{\prime}=1$, then either $\left(^{*}\right)$ holds or $k_{0}=-(a+1)^{-1}, k_{1}=k_{2}=k_{3}=0$,

(iv) $a \in \mathbb{N}$ and $k_{-}^{\prime}=1$, then either $\left(^{*}\right)$ holds or $k_{0}=-a(a+1)^{-1}, k_{1}=k_{2}=k_{3}=0$,

$F(4)$ : one of the two possibilities holds:

(i) $k^{\prime}=0$, then all $k_{i}=0$,

(ii) $k^{\prime}=1$, then $k_{0}=-\frac{2}{3}, k_{1}=k_{2}=k_{3}=k_{4}=0$,

$G(3)$ : one of the two possibilities holds:

(i) $k^{\prime}=0$, then all $k_{i}=0$,

(ii) $k^{\prime}=1$, then $k_{0}=-\frac{3}{4}, k_{1}=k_{2}=k_{3}=0$,

(4) if $k^{\prime \prime} \leq b^{\prime \prime}$ (see Table Њ), there are the supplementary conditions:

$$
B(m, n): k_{j}=0 \text { for all } j \geq n+k^{\prime \prime}+1,
$$


$C(n) \widetilde{R} k_{0}=k_{1}=0$,

$D(m, n)$ : one of the two possibilities holds:

(i) $k^{\prime \prime} \leq m-2$, then $k_{j}=0$ for all $j \geq n+k^{\prime \prime}+1$,

(ii) $k^{\prime \prime}=m-1$, then $k_{m+n-1}=k_{m+n}$,

$D(2,1 ; a)$ : one of the two possibilities holds:

(i) $k^{\prime \prime}=0$, then $k_{1}=k_{2}=k_{3}=0$,

(ii) $k^{\prime \prime}=1$, then $a \in \mathbb{Q}$ and $k_{2}+1=|a|\left(k_{3}+1\right)$,

$F(4)$ : one of the three possibilities holds:

(i) $k^{\prime \prime}=0$, then $k_{1}=k_{2}=k_{3}=k_{4}=0$,

(ii) $k^{\prime \prime}=2$, then $k_{2}=k_{4}=0$,

(iii) $k^{\prime \prime}=3$, then $k_{2}=2 k_{4}+1$,

$G(3)$ : one of the two possibilities holds:

(i) $k^{\prime \prime}=0$, then $k_{1}=k_{2}=k_{3}=0$,

(ii) $k^{\prime \prime}=2$, then $k_{2}=0$.

Proof. In the case $\widehat{\mathfrak{g}}=A(m, n)$, the theorem follows from Theorem 2.1. In general, the proof is based on similar arguments. Below we shall give details in the case $\widehat{\mathfrak{g}}=B(1, n) \hat{\text {; }}$ in the rest of the cases arguments are the same.

The even part of $B(1, n)$ is $A_{1}+C_{n}$ and its simple roots are $\alpha_{n+1}$ for $A_{1}$ and $\left\{\alpha_{1}, \alpha_{2}, \ldots, \alpha_{n-1}\right.$, $\left.\alpha^{\prime \prime}=2\left(\alpha_{n}+\alpha_{n+1}\right)\right\}$ for $C_{n}$. The simple roots of $\widehat{A}_{1}$ are $\left\{\alpha_{0}^{\prime}=\delta-\alpha_{n+1}, \alpha_{n+1}\right\}$. Due to Lemma 1.5a, the local finiteness (resp. integrability) with respect to $C_{n}$ (resp. $\widehat{A}_{1}$ ) implies that $k_{1}, \ldots, k_{n-1}, k^{\prime \prime} \in \mathbb{Z}_{+}$(resp. $k_{n+1}, k^{\prime} \in \mathbb{Z}_{+}$). Hence, conditions (1) and (2) are necessary. Furthermore, it follows from Proposition 1.2, that in the cases $k^{\prime}>b^{\prime}=4 n-1$ (resp. $k^{\prime \prime}>b^{\prime \prime}=\frac{1}{2}$ ) the element $f^{\prime}$ (resp. $f^{\prime \prime}$ ) is locally nilpotent. It remains to show that in the case of inequality

$$
k^{\prime} \leq 4 n-1
$$

the element $f^{\prime}$ is locally nilpotent iff condition (3) holds, and in the case $k^{\prime \prime}=0, f^{\prime \prime}$ is locally nilpotent iff (4) holds. We shall concentrate on the first claim, the second being easier (cf. also [K1]).

Introduce the following isotropic roots:

$$
\beta_{j}=\sum_{i=n-j}^{n} \alpha_{i}(j=0, \ldots, n), \beta_{n+j}=\beta_{n}+\sum_{i=1}^{j} \alpha_{i}(j=1, \ldots, n-1) .
$$

We have: $\beta_{j}^{\vee}=\beta_{j}$ for all $j$, and

$$
\left(\beta_{i} \mid \alpha_{n+1}\right)=-1 \text { for all } i,\left(\beta_{i} \mid \beta_{i+1}\right)=\left\{\begin{array}{lll}
1 & \text { if } & 0 \leq i \leq n-2 \\
2 & \text { if } & i=n-1
\end{array}\right.
$$


Let $\Pi^{(0)}=\Pi$, then $\beta_{0} \in \Pi^{(0)}$ and we let $\Pi^{(1)}=r_{\beta_{0}} \Pi^{(0)}$. Similarly $\beta_{1} \in \Pi^{(1)}$ and we let $\Pi^{(2)}=r_{\beta_{1}} \Pi^{(1)}, \ldots, \Pi^{(2 n)}=r_{\beta_{2 n-1}} \Pi^{(2 n-1)}$. We have:

$$
\alpha_{0}^{\prime} \in \Pi^{(2 n)}, \alpha_{0}^{\prime \vee}=2 \alpha_{0}^{\prime}
$$

Let $\Lambda^{(j)}$ denote the highest weight of $L(\Lambda)$ with respect to $\mathfrak{n}_{+}^{(j)}$. It can be computed by making use of Lemma 1.4. Introduce the following numbers:

$$
u_{j}=\left\langle\Lambda^{(j)}, \alpha_{0}^{\prime \vee}\right\rangle(j=0, \ldots, 2 n), \quad t_{j}=\left\langle\Lambda^{(j)}, \beta_{j}\right\rangle(j=0, \ldots, 2 n-1) .
$$

Using (6.2), $\left\langle\Lambda, \alpha_{0}^{\prime \vee}\right\rangle=k^{\prime}$ and Lemma 1.4, we get the following recurrent formula for the $u_{j}$ 's:

$$
u_{0}=k^{\prime}, u_{j+1}=u_{j}-2\left(\operatorname{resp} .=u_{j}\right) \text { if } t_{j} \neq 0(\text { resp. }=0) .
$$

In view of Lemma 1.5, the local nilpotency of $f^{\prime}$ follows from $u_{2 n} \in \mathbb{Z}_{+}$. This, clearly, holds if $k^{\prime} \geq 4 n$ (by (6.4)), which again shows that in this case there are no supplementary conditions.

¿From now on we may assume that $k^{\prime} \leq 4 n-1$. We may also assume that conditions (1), (2) and (4) hold. We shall derive a recurrent formula for the $t_{i}$. Using that, by Lemma 1.4, $\Lambda^{(i+1)}=\Lambda^{(i)}-\beta_{i}\left(\right.$ resp. $\left.=\Lambda^{(i)}\right)$ if $t_{i} \neq 0($ resp. $=0)$ and that $\alpha_{n-i-1} \in \Pi^{(i)}$, we obtain:

$$
\begin{gathered}
t_{i+1}=\left\{\begin{array}{ll}
t_{i}-k_{n-i-1}-1 & \text { if } t_{i} \neq 0 \\
t_{i}-k_{n-i-1} & \text { if } t_{i}=0
\end{array} \quad(0 \leq i \leq n-2)\right. \\
t_{n}=t_{n-1}-2 k_{0}-2\left(\text { resp. }=t_{n-1}-2 k_{0}\right) \text { if } t_{n-1} \neq 0(\text { resp. }=0) .
\end{gathered}
$$

For $t_{j}, j \geq n$, the recurrent formula involves numbers

$$
s_{i}=\left(\Lambda^{(n)} \mid \alpha_{i}\right) \text { for } 1 \leq i \leq n-1, s_{n}=\left(\Lambda^{(n)} \mid \alpha_{n}+\alpha_{n+1}\right),
$$

which, using the above arguments, can be expressed in terms of the labels of $\Lambda$ as follows:

$$
s_{i}= \begin{cases}-k_{i} & \text { if } \quad t_{n-i-1} t_{n-i} \neq 0 \text { or } t_{n-i-1}=t_{n-i}=0 \quad(1 \leq i \leq n-1), \\ -k_{i}-1 & \text { if } t_{n-i-1} \neq 0, t_{n-i}=0, \\ -k_{i}+1 & \text { if } \quad t_{n-i-1}=0, t_{n-i} \neq 0,\end{cases}
$$

$$
s_{n}=-k^{\prime \prime}+1\left(\text { resp. }-k^{\prime \prime}\right) \text {, where } k^{\prime \prime}=-k_{n}-\frac{1}{2} k_{n+1}, \text { if } k_{n} \neq 0(\text { resp. }=0) .
$$

Then we have $(0 \leq i \leq n-1)$ :

$$
t_{n+i+1}=t_{n+i}+s_{i+1}-1\left(\text { resp. } t_{n+i}+s_{i+1}\right) \text { if } t_{n+i} \neq 0(\text { resp. }=0),
$$

where we let

$$
t_{2 n}=\frac{1}{2} u_{2 n}
$$


Note that $t_{0}=k_{n} \leq 0$ since

$$
k^{\prime \prime}=-k_{n}-\frac{1}{2} k_{n+1} \in \mathbb{Z}_{+} \text {and } k_{n+1} \in \mathbb{Z}_{+} .
$$

Since $k_{i} \in \mathbb{Z}_{+}$for $i \neq 0, n$, formulae (6.5) imply

$$
0 \geq t_{0} \geq t_{1} \geq \cdots \geq t_{n-1}
$$

Furthermore, we have

$$
t_{n} \geq t_{n+1} \geq \cdots \geq t_{2 n}=\frac{1}{2} u_{2 n} .
$$

In order to show this, it suffices to prove that $s_{i} \leq 0,1 \leq i \leq n$. But, due to 6.6a), $s_{i}>0$ can take place for $1 \leq i \leq n-1$ only when $t_{n-i-1}=0, t_{n-i} \neq 0, k_{i}=0$, which is impossible by (6.5a). Also, $s_{n}=-k^{\prime \prime}+1$ (resp. $\left.-k^{\prime \prime}\right)$ if $k_{n} \neq 0($ resp. $=0)$ cannot be positive since in this case $k^{\prime \prime}=0$, which implies that $k_{n}=0$ (see supplementary conditions (4)).

Suppose now that $f^{\prime}$ is locally nilpotent. Then $u_{2 n} \geq 0$ (by Lemma 1.5), hence we have from (6.4):

$$
t_{j}=0 \text { for some } 0 \leq j \leq 2 n-1 .
$$

Due to (6.10), (6.8) and (6.9), we have the following three possibilities for some $0 \leq i_{0} \leq n-1$ and $n \leq j_{0} \leq 2 n-1$ :

( $\alpha) \quad t_{0}=\cdots=t_{i_{0}}=0, t_{i_{0}+1} \neq 0, \ldots, t_{2 n-1} \neq 0$

(及) $t_{0} \neq 0, \ldots, t_{j_{0}-1} \neq 0, t_{j_{0}}=\cdots=t_{2 n}=0$,

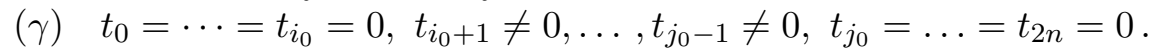

The possibilities (i), (ii), (iii) and (iv) of the supplementary conditions (3) correspond respectively to the following cases:

(i) $(\gamma)$ when $i_{0}+j_{0}<2 n-1$, where we put $i_{0}=n-s-1, j_{0}=n+r$,

(ii) $(\gamma)$ when $i_{0}+j_{0} \geq 2 n-1$, where we put $i_{0}=n-r-1, j_{0}=n+s$,

(iii) $(\beta)$, where we put $j_{0}=n+r$,

(iv) $(\alpha)$, where we put $i_{0}=n-r-1$.

We consider in detail only case (ii), the treatment of all other cases being similar. We have:

$$
t_{0}=\cdots=t_{n-r-1}=0, t_{n-r} \neq 0, \ldots, t_{n+s-1} \neq 0, t_{n+s}=\cdots=t_{2 n-1}=0
$$

for some integer $s$ such that $0 \leq r \leq s \leq n-1$. Hence, by (6.5a) we have:

$$
k_{r} \neq 0, k_{r+1}=\cdots=k_{n}=0
$$

and, by (6.6a), we have:

$$
s_{i}=-k_{i} \text { if } i \neq r, 1 \leq i \leq n-1, s_{r}=-k_{r}+1, s_{n}=0 .
$$


The recurrent formulas $(6.5)$ and $(6.7)$ can be now rewritten respectively as follows:

$$
\begin{aligned}
t_{n-r} & =-k_{r} \\
t_{n-r+1} & =t_{n-r}-k_{r-1}-1 \\
\cdots & \\
t_{n-1} & =t_{n-2}-k_{1}-1 \\
t_{n} & =t_{n-1}-2 k_{0}-2 \\
t_{n+1} & =t_{n}-k_{1}-1 \\
\cdots & \\
t_{n+r-1} & =t_{n+r-2}-k_{r-1}-1 \\
t_{n+r} & =t_{n+r-1}-k_{r} \\
t_{n+r+1} & =t_{n+r}-k_{r+1}-1 \\
\cdots & \\
t_{n+s} & =t_{n+s-1}-k_{s}-1+\delta_{r, s} .
\end{aligned}
$$

Summing up these equalities, we get: $2 \sum_{i=0}^{r} k_{i}=-(r+s)$, which, in view of (6.11), implies $k^{\prime}=2(r+s)$.

Suppose now that conditions (1)-(4) hold. We have to show that $u_{2 n} \geq 0$. As before, we may assume that $k^{\prime} \leq 4 n-1$, hence, due to (3), (6.10) holds. Since (6.8) and (6.9) hold, we again have only the possibilities $(\alpha),(\beta)$ and $(\gamma)$. In cases $(\beta)$ and $(\gamma), u_{2 n}=2 t_{2 n}=0$, hence only case $(\alpha)$ remains. This case corresponds to (3)iv when we have: $t_{0}=k_{n}=0$, $t_{1}=t_{0}-k_{n-1}=0, \ldots, t_{n-r-1}=t_{n-r-2}-k_{r+1}=0$. Hence $v:=\#\left\{0 \leq j \leq 2 n-1 \mid t_{j} \neq 0\right\} \leq n+r$ and $u_{2 n}=u_{0}-2 v \geq k^{\prime}-2(n+r) \geq 0$ (we have used here (6.4) and (3)iv).

Theorem 6.2. For an affine superalgebra $\widehat{\mathfrak{g}}$ from Table $₫$ the labels $\left\{k_{i}\right\}_{i \in \hat{I}}$ of the highest weight of a subprincipal integrable irreducible highest weight module are characterized by the following conditions:

(1) $k_{i} \in \mathbb{Z}_{+}$if $i \in \widehat{I} \backslash I_{1}, k^{\prime \prime} \in \mathbb{Z}_{+}$,

(2) if $k^{\prime \prime} \leq b^{\prime \prime}$ (see Table (4), there are supplementary conditions described by (4) of Theorem 6.1 .

Proof. The only simple root of $\widehat{\mathfrak{g}}_{0}^{\prime \prime}$ which is not simple for $\widehat{\mathfrak{g}}$ is $\alpha^{\prime \prime}$. Hence the proof of Theorem 6.1 proves Theorem 6.2 as well.

Remark 6.2. It follows from Theorem 6.1 that the level $k$ of a principal integrable $\widehat{\mathfrak{g}}$-module $L(\Lambda)$ is a non-negative number which is an integer in all cases, except for $B(1, n)$, when it is a half-integer; moreover, if $k=0$, then all labels of $\Lambda$ are 0 , hence $L(\Lambda)$ is 1-dimensional; also, if $k>0$, then $k \geq 1$.

Remark 6.3. It is easy to see that, when restricted to the derived subalgebra $[\widehat{\mathfrak{g}}, \widehat{\mathfrak{g}}]$ of $\widehat{\mathfrak{g}}$ the module $L(\Lambda)$ remains irreducible. Two $\widehat{\mathfrak{g}}$-modules are called essentially equivalent if they are equivalent as $[\widehat{\mathfrak{g}}, \widehat{\mathfrak{g}}]$-modules. For example, the modules $L(\Lambda)$ and $L(\Lambda+a \delta)$ are essentially equivalent for any $a \in \mathbb{C}$. Theorem 6.1 gives the following complete list of principal integrable modules of level 1 up to essential equivalence: 
(1) $A(m, n), m \geq 1: \Lambda_{s}(1 \leq s \leq m),(a+1) \Lambda_{m+1}+a \Lambda_{m+2}\left(a \in \mathbb{Z}_{+}\right)$, and $(a+1) \Lambda_{0}+$ $a \Lambda_{m+n+1}\left(a \in \mathbb{Z}_{+}\right)$,

(2) $B(m, 1)$ and $D(m, 1)$ : $-\frac{1}{2} \Lambda_{0}$ and $-\frac{3}{2} \Lambda_{0}-\Lambda_{1}$,

(3) $B(m, n)$ and $D(m, n) \hat{)}, n \geq 2:-\frac{1}{2} \Lambda_{0}$ and $-\frac{3}{2} \Lambda_{0}+\Lambda_{1}$,

(4) $D(2,1 ; a), a^{-1} \in \mathbb{N}:-(a+1)^{-1} \Lambda_{0}$ and $-\frac{a+2}{a+1} \Lambda_{0}-\Lambda_{1}+\frac{1-a}{a} \Lambda_{3} a^{-1}$,

(5) $F(4) \hat{:}-\frac{2}{3} \Lambda_{0}$,

(6) $G(3) \widehat{:}-\frac{3}{4} \Lambda_{0}$.

One can show (cf. Remark 2.5) that in all cases, all weights are conjugate to each other by odd reflections. Thus, for each of the affine superalgebras $A(m, n) \hat{(}(m \geq 1), B(m, n) \hat{,} D(m, n)$, $D(2,1 ; a)\left(a \in \mathbb{Q}_{>}\right), F(4) \hat{\text { and }} G(3)$ all, up to essential equivalence, principal integrable modules of level 1, can be obtained from one of them by making different choices of the set of positive roots. Note also that in all cases the "basic" module $L\left(u \Lambda_{0}\right)$, where $u$ is such that $u \Lambda_{0}$ has level 1 , is a principal integrable module.

Remark 6.4. Using the symmetry of $A(m, n)$ which exchanges the subalgebras $\widehat{A}_{m}$ and $\widehat{A}_{n}$, one gets the classification of the subprincipal integrable modules $L(\Lambda)$ for this affine subalgebra:

$$
k_{i} \in \mathbb{Z}_{+} \text {for } i \in \widehat{I} \backslash \widehat{I}_{1}, \quad k^{\prime \prime}:=-\sum_{i=0}^{m+1} k_{i} \in \mathbb{Z}_{+},
$$

and there exists $s \in \mathbb{Z}_{+}, s \leq k^{\prime \prime}$, such that

$$
k_{0}+k_{1}+\cdots+k_{s}+s=0 \text { and } k_{s+1}=\cdots=k_{s+m-k^{\prime \prime}+1}=0 .
$$

One has: $k=-\left(k^{\prime \prime}+\sum_{i=n+2}^{m+n+1} k_{i}\right)$.

Remark 6.5. All principal integrable highest weights of level 2 (up to essential equivalence) for $B(1,2)$ are $-(1+a) \Lambda_{0}+a \Lambda_{1}$, where $a \in \mathbb{Z}_{+}$. Thus, in sharp contrast to the level 1 case, there are infinitely many essentially inequivalent principal integrable highest weight modules of level $\geq 2$.

Remark 6.6. It follows from Theorem 6.2 and Remark 6.3 that the level $k$ of a subprincipal integrable $\widehat{\mathfrak{g}}$-module $L(\Lambda)$ is a non-positive number, provided that $a>-1$ for $D(2,1 ; a)$; moreover, $\operatorname{dim} L(\Lambda)=1$ if $k=0$. Thus, in view of Theorem 6.1, the only $L(\Lambda)$ over $\widehat{\mathfrak{g}} \neq A(m, 0)$, $A(0, n)$ or $C(n) \hat{)}$, which are integrable over $\widehat{\mathfrak{g}_{\overline{0}}}$ are 1-dimensional.

Remark 6.7. Using the same arguments, one can show that the non-symmetrizable "twisted" affine superalgebra of type $Q$ (which is the universal central extension of the Lie superalgebra 


$$
\begin{aligned}
& \left.\sum_{n \in \mathbb{Z}}\left(Q(n)_{\overline{0}} t^{2 n}+Q(n)_{\overline{1}} t^{2 n+1}\right)\right), \text { with the Cartan matrix } \\
& \qquad\left(\begin{array}{ccccc}
0 & 1 & 0 & \cdots & -1 \\
-1 & & & & \\
0 & & & A_{n} & \\
\cdots & & & \\
0 & & & \\
-1 & &
\end{array}\right)
\end{aligned}
$$

has no non-trivial integrable (with respect to its even part) highest weight modules.

Remark 6.8. Consider the $\mathbb{Z} / 2 \mathbb{Z}$-gradation of $F(4)$ of type $(0,0,0,1,0)$ and that of $G(3)$ of type $(0,0,0,1)$, cf. Table 1 and [K3]. The $0^{\text {th }}$ piece in the first (resp. second) case is isomorphic to $D(2,1 ; 1 / 2) \oplus A_{1}$ (resp. to $D(2,1 ; 1 / 3)$ ), and its representation on the $1^{\text {st }}$ piece is the module $\mathbb{C}^{10} \otimes \mathbb{C}^{2}$ (resp. $\mathbb{C}^{14}$ ), where $\mathbb{C}^{10}$ (resp. $\mathbb{C}^{14}$ ) is the lowest-dimensional non-trivial module over $D(2,1 ; 1 / 2)$ (resp. $D(2,1 ; 1 / 3)$ ). This reduces to some extent the construction of the principal integrable level 1 module over $F(4)$ and $G(3)$ to that of $D(2,1 ; a)$. The free field construction of the principal integrable level 1 modules over $\operatorname{osp}(m, n)$ (covering the $B-C-D$ cases) will be given in Section 7 .

\section{Free field realization of level 1 integrable modules over $\operatorname{csp}(M \mid N)^{\uparrow}$.}

Let $V$ be the superspace and let (.|.) be the bilinear form on $V$ considered in Remark 6.1. Recall an equivalent definition of $\operatorname{ssp}(M \mid N)$ via the Clifford superalgebra:

$$
C \ell V=T(V) /\langle[x, y]-(x \mid y) 1 \mid x, y \in V\rangle .
$$

The Lie superalgebra $\operatorname{csp}(M \mid N)$ is identified with the $\mathbb{C}$-span of all quadratic elements of $C \ell V$ of the form:

$$
: \alpha \beta: \equiv \alpha \beta+(-1)^{p(\alpha) p(\beta)} \beta \alpha, \quad \text { where } \alpha, \beta \in V .
$$

Such an element is identified with an operator from $\operatorname{osp}(M \mid N)$ by the formula:

$$
(: \alpha \beta:) v=[: \alpha \beta:, v], \quad v \in V .
$$

Denote by $\Phi_{V}$ the vertex algebra generated by pairwise local fields $\gamma(z)$, where $\gamma \in V_{\overline{0}} \cup V_{\overline{1}}$ and $\gamma(z)$ is even (resp. odd) if $\gamma \in V_{\overline{0}}$ (resp. $V_{\overline{1}}$ ), subject to the following OPE:

$$
\gamma(z) \gamma^{\prime}(\omega) \sim \frac{\left(\gamma \mid \gamma^{\prime}\right)}{z-w}
$$

This is called the vertex algebra of free superfermions in [K4].

Remark 7.1. The vertex algebra $F$ considered in $\S 3$ is isomorphic to $\Phi_{V}$, where $\operatorname{dim} V_{\overline{0}}=$ $2 n, \operatorname{dim} V_{\overline{1}}=2 m$ and the bilinear form is given by:

$$
\begin{aligned}
\left(\varphi^{i *} \mid \varphi^{j}\right) & =-\left(\varphi^{i} \mid \varphi^{j *}\right)=\delta_{i j}(i, j=1, \ldots, n), \\
\left(\psi^{i *} \mid \psi^{j}\right) & =\left(\psi^{i} \mid \psi^{j *}\right)=\delta_{i j}(i, j=1, \ldots, m), \text { all other inner products }=0 .
\end{aligned}
$$


Furthermore, in the case when $\operatorname{dim} V_{\overline{1}}=2 m+1$ the vertex algebra $\Phi_{V}$ is isomorphic to $F \otimes \Phi$, where $\Phi$ is a vertex algebra generated by one odd field $\psi(z)$ with the OPE $\psi(z) \psi(w) \sim \frac{1}{z-w}$. This corresponds to adding an odd vector $\psi$ with $(\psi \mid \psi)=1$ orthogonal to all the above basis vectors.

As in $\S$ 3, we construct the Virasoro field $L(z) \equiv \sum_{j \in \mathbb{Z}} L_{j} z^{-j-2}$ with respect to which all $\gamma(z)$ are primary of conformal weight $1 / 2$. Choose a basis $\varphi^{i}, \varphi^{i *}(i=1, \ldots, n)$ of $V_{\overline{0}}$, and a basis $\psi^{i}, \psi^{i *}(i=1, \ldots, m)$ and $\psi$ if $M$ is odd, with inner products described by Remark 7.1. Then $L(z)$ is given by formula (3.1) if $M$ is even. In the case $M$ is odd, one should add to the expression (3.1) the term $\frac{1}{2}: \partial \psi(z) \psi(z):$ As in $\S$ 3, we shall write $\gamma(z)=\sum_{k \in \frac{1}{2}+\mathbb{Z}} \gamma_{k} z^{-k-1 / 2}, \gamma \in V_{\overline{0}} \cup V_{\overline{1}}$.

We shall need also the following well-known fact (see e.g. [K4], formula (5.1.5)).

Lemma 7.1. Let $\psi^{+}, \psi^{-} \in V_{\overline{1}}$ be such that $\left(\psi^{ \pm} \mid \psi^{ \pm}\right)=0,\left(\psi^{+} \mid \psi^{-}\right)=1$. Let $\alpha(z) \equiv \sum_{n \in \mathbb{Z}} \alpha_{n} z^{-n-1}$ $=: \psi^{+}(z) \psi^{-}(z)$ :. Then one has:

$$
: \alpha(z) \alpha(z):=: \partial \psi^{+}(z) \psi^{-}(z):+: \partial \psi^{-}(z) \psi^{+}(z): \text {. }
$$

Consequently, the fields $\psi^{ \pm}(z)$ are primary of conformal weight $1 / 2$ with respect to the Virasoro field $\ell(z) \equiv \sum_{n \in \mathbb{Z}} \ell_{n} z^{-n-2}=\frac{1}{2}: \alpha(z) \alpha(z)$ :. In particular, we have

$$
\left[\ell_{0}, \psi_{n}^{ \pm}\right]=-n \psi_{n}^{ \pm}
$$

Note that

$$
\gamma_{j}|0\rangle=0 \text { for } j>0, \gamma \in V \text {. }
$$

Hence $\Phi_{V}$ is obtained by applying polynomials in the $\gamma_{-j}, \gamma \in V, j>0$, to the vacuum vector $|0\rangle$. We have the decomposition

$$
\Phi_{V}=\Phi_{V}^{+} \oplus \Phi_{V}^{-}
$$

where $\Phi_{V}^{+}$(resp. $\left.\Phi_{V}^{-}\right)$is obtained by applying even (resp. odd) degree polynomials in the $\gamma_{-j}$ to $|0\rangle$.

Theorem 7.1. (a) Consider the affine superalgebra osp $(M \mid N)^{\Upsilon}$ and let : $\alpha \beta$ : $(z)=$ $\sum_{k \in \mathbb{Z}}\left(t^{k} \otimes: \alpha \beta:\right) z^{-k-1}$ for $: \alpha \beta: \in \operatorname{osp}(M \mid N)$. Then the linear map $\sigma$ given by $(\alpha, \beta \in V)$ :

$$
: \alpha \beta:(z) \mapsto: \alpha(z) \beta(z):, K \mapsto 1, d \mapsto L_{0}
$$

defines a principal integrable representation of osp $(M \mid N)^{\Upsilon}$ of level 1 in the space $\Phi_{V}$ for which $\Phi_{V}^{+}$and $\Phi_{V}^{-}$are submodules.

(b) The $\operatorname{osp}(M \mid N)^{-}$-modules $\Phi_{V}^{+}$and $\Phi_{V}^{-}$are irreducible highest weight modules isomorphic to $L\left(-\frac{1}{2} \Lambda_{0}\right)$ and $L\left(-\frac{1}{2} \Lambda_{0}-\frac{1}{2} \alpha_{0}\right)$ respectively, provided that $(M, N) \neq(1,0)$ or $(2,0)$. 
Proof. The proof that $\sigma$ is a representation is, as usual, a straightforward use of Wick's formula. The proof of integrability of $\sigma$ is the same as in the proof of Theorem 3.1. This establishes (a).

Note that, as before, $L_{0}$ commutes with $\operatorname{osp}(M \mid N)$, and the spectrum of $L_{0}$ on $\Phi_{V}^{+}$(resp. $\Phi_{V}^{-}$) is $\mathbb{Z}_{+}$(resp. $\frac{1}{2}+\mathbb{Z}_{+}$), the lowest eigenvalue eigenspace being $S^{ \pm}=\mathbb{C}|0\rangle$ (resp. $S^{-}=$ $\left\{\gamma_{-\frac{1}{2}}|0\rangle \mid \gamma \in V\right\}$ ), which is the trivial 1-dimensional (resp. the standard) representation of $\operatorname{osp}(M \mid N)$. Provided that $\Phi_{V}^{ \pm}$are irreducible $\operatorname{ssp}(M \mid N)$-modules, (b) follows.

In order to prove irreducibility of $\Phi_{V}^{ \pm}$, pick elements $\psi^{+}, \psi^{-} \in V_{\overline{1}}$ as in Lemma 7.1 and define the field $\ell(z)$ as in that lemma. Let $\psi \in V_{\overline{1}}$ be an element orthogonal to both $\psi^{+}$and $\psi^{-}$, and consider the field $\beta(z)=: \psi^{+}(z) \psi(z): \equiv \sum_{n \in \mathbb{Z}} \beta_{n} z^{-n-1}$, so that $\beta_{n}=\sum_{j \in \frac{1}{2}+\mathbb{Z}}: \psi_{j}^{+} \psi_{n-j}:$ Since $\ell_{0}$ commutes with $\psi(z)$, we have by $(7.2)$ :

$$
\left[\ell_{0}, \beta_{n}\right]=\sum_{j \in \frac{1}{2}+\mathbb{Z}} j: \psi_{j}^{+} \psi_{n-j}:
$$

Let $U \subset \Phi_{V}^{ \pm}$be an invariant with respect to $\operatorname{osp}(M \mid N)$ subspace. It follows from Lemma 7.1 and (7.4) that $v \in U$ implies that $\left(\left(\operatorname{ad} \ell_{0}\right)^{s} \beta_{n}\right) v \in U, s \in \mathbb{Z}_{+}$. Hence $U$ is invariant with respect to all operators $\psi_{j}^{+} \psi_{k}$, where $\psi^{+}, \psi \in V$ are such that $\left(\psi^{+} \mid \psi^{+}\right)=0=\left(\psi^{+} \mid \psi\right)$ and $j, k \in \frac{1}{2}+\mathbb{Z}$.

Hence, provided that $M \geq 3, U$ contains a non-zero purely bosonic element, i.e., an element obtained by applying a polynomial in the $\gamma_{j}\left(\gamma \in V_{\overline{0}}\right)$ to $|0\rangle$. Thus we reduced the problem to the purely bosonic case, i.e., the case when $M=0$. In this case the irreducibility was proved in [4] using the character formula for modular invariant representations of $\widehat{C}_{n}$ from [KW1] and formula (12.13) from [K3] (the reference to (13.13) in $[\mathrm{D}]$ is a misprint).

The remaining cases, when $M=1$ or 2 and $N=2 n$ is even $\geq 2$ can be reduced again to the purely bosonic case by a direct calculation. We give below details in the $M=1$ case, the $M=2$ case being similar.

The simple root vectors of $\operatorname{osp}(1, N)^{\Upsilon}=B(0, n)$ are as follows:

$$
\begin{aligned}
& e_{0}=\left(\varphi^{1 *}(z) \varphi^{1 *}(z)\right)_{1}=\sum_{s \in \mathbb{Z}} \varphi_{-s+1 / 2}^{(1) *} \varphi_{s+1 / 2}^{(1) *}, \\
& e_{i}=\left(\varphi^{i}(z) \varphi^{i+1 *}(z)\right)_{0}=\sum_{s \in \mathbb{Z}} \varphi_{-s-1 / 2}^{(i)} \varphi_{s+1 / 2}^{(i+1) *} \quad(i=1, \ldots, n-1), \\
& e_{n}=(\varphi(z) \psi(z))_{0} \quad=\sum_{s \in \mathbb{Z}} \varphi_{-s-1 / 2}^{(n)} \psi_{s+1 / 2} \text {. }
\end{aligned}
$$

Then the simple root vectors of $s p(N)^{\hat{\gamma}}=\widehat{C}_{n}$ are $e_{0}, e_{1}, \ldots e_{n-1}$ and $e_{n}^{\prime}=\left[e_{n}, e_{n}\right]=$ $\sum_{s \in \mathbb{Z}} \varphi_{-s-1 / 2}^{(n)} \varphi_{s+1 / 2}^{(n)}$.

Any vector $v$ of $\Phi_{V}$ can be uniquely written in the form:

$$
v=\sum_{i_{1}<\cdots<i_{k}} \psi_{i_{1}} \ldots \psi_{i_{k}} u_{i_{1}, \ldots, i_{k}}
$$

where $u_{i_{1}, \ldots, i_{k}}$ are purely bosonic elements (i.e., obtained by applying polynomials in the $\varphi$ 's to $|0\rangle)$. Now, if $v$ is a singular vector, i.e., $e_{i} v=0$ for all $i=0, \ldots, n$, then, in particular, $e_{n}^{\prime} v=0$, and since all $e_{1}, \ldots, e_{n-1}, e_{n}^{\prime}$ commute with the $\psi$ 's, we get:

$$
\sum_{i_{1}<\cdots<i_{k}} \psi_{i_{1}} \ldots \psi_{i_{k}}\left(e_{i} u_{i_{1}, \ldots, i_{k}}\right)=0, \sum_{i_{1}<\cdots<i_{k}} \psi_{i_{1}} \ldots \psi_{i_{k}}\left(e_{n}^{\prime} u_{i_{1}, \ldots, i_{n}}\right)=0
$$


It follows that all $u_{i_{1}, \ldots, i_{n}}$ are purely bosonic singular with respect to $\widehat{C}_{n}$ vectors, hence, due to irreducibility of $\Phi_{V}^{ \pm}$for $M=0$ mentioned above, we obtain that all $u_{i_{1} \ldots i_{k}}$ are linear combinations of elements $|0\rangle$ and $\varphi_{-1 / 2}^{(1)}|0\rangle$. Hence

$$
v=\sum_{i_{1}<\cdots<i_{k}} a_{i_{1}, \ldots, i_{k}} \psi_{i_{1}} \ldots \psi_{i_{k}}|0\rangle+\sum_{i_{1}<\cdots<i_{k}} b_{i_{1}, \ldots, i_{k}} \psi_{i_{1}} \ldots \psi_{i_{k}} \varphi_{-1 / 2}^{(1)}|0\rangle
$$

Using that $e_{n} v=0$, we obtain:

$$
\begin{array}{r}
\sum_{r=1}^{k} \sum_{i_{1}<\cdots<i_{k}}(-1)^{r-1} a_{i_{1}, \ldots, i_{k}} \psi_{i_{1}} \ldots \widehat{\psi}_{i_{r}} \ldots \psi_{i_{k}} \varphi_{i_{r}}^{(n)}|0\rangle \\
+\sum_{r=1}^{k} \sum_{i_{1}<\ldots<i_{k}}(-1)^{r-1} b_{i_{1}, \ldots, i_{k}} \psi_{i_{1}} \ldots \widehat{\psi}_{i_{r}} \ldots \psi_{i_{k}} \varphi_{i_{r}}^{(n)} \varphi_{-1 / 2}^{(1)}|0\rangle=0
\end{array}
$$

which implies that $a_{i_{1}, \ldots, i_{k}}$ (resp. $\left.b_{i_{1}, \ldots, i_{k}}\right)=0$ if $k>0$. Thus, the only singular vectors in $\Phi_{V}^{+}$ (resp. $\left.\Phi_{V}^{-}\right)$are scalar multiples of $|0\rangle$ (resp. $\left.\varphi_{-1 / 2}^{(1)}|0\rangle\right)$.

To conclude that the $B(0, n)$-modules $\Phi_{V}^{ \pm}$are irreducible, note that $\Phi_{V}$ carries a unique non-degenerate Hermitian form $H(.,$.$) such that the square length of |0\rangle$ is 1 and the adjoint operators of $\varphi_{k}^{(j)}$ and $\psi_{k}$ are $\varphi_{-k}^{(j) *}$ and $\psi_{-k}$, respectively. The absence of non-trivial singular vectors in $\Phi_{V}^{+}$(resp. $\left.\Phi_{V}^{-}\right)$implies that the $B(0, n)$-submodules $\Phi_{V}^{+\prime}$ (resp. $\Phi_{V}^{-\prime}$ ) generated by $|0\rangle$ (resp. $\varphi_{-1 / 2}^{(1)}|0\rangle$ ) is irreducible, hence the restriction of $H$ to it is non-degenerate. Hence the orthogonal complement to $\Phi_{V}^{+\prime}$ (resp. $\Phi_{V}^{-\prime}$ ) is a complementary submodule which has no non-zero singular vectors, hence it is zero, and $\Phi_{V}^{ \pm}$are irreducible.

Remark 7.2. The irreducibility in the purely fermionic case was established in KP1 by making use of the Weyl-Kac character formula. An argument, using Virasoro operators, was given in $[$ F]. The method of using Virasoro operators to prove irreducibility apparently works only in the presence of fermions (cf. Remark 3.3). It is shown in [D that the irreducibility claims of [FF], based on the use of Virasoro operators, are false for the constructions of $A_{2 \ell-1}^{(2)}$ and $A_{2 \ell}^{(2)}$-modules.

Using Theorem 7.1, it is straightforward to write down the characters and supercharacters for the integrable level $1 \operatorname{osp}(M \mid N)$-modules. We have:

$$
\operatorname{ch} \Phi_{V}^{+} \pm \operatorname{ch} \Phi_{V}^{-}=e^{-\frac{1}{2} \Lambda_{0}} \Pi_{k=1}^{\infty} \frac{\left(1 \pm q^{k-1 / 2}\right)^{p(M)} \Pi_{i=1}^{m}\left(1 \pm e^{\epsilon_{i}} q^{k-1 / 2}\right)\left(1 \pm e^{-\epsilon_{i}} q^{k-1 / 2}\right)}{\Pi_{j=1}^{n}\left(1 \mp e^{\epsilon_{j+m}} q^{k-1 / 2}\right)\left(1 \mp e^{-\epsilon_{j+m}} q^{k-1 / 2}\right)}
$$

where $p(M)=0$ (resp. 1) if $M$ is even (resp. odd). A similar formula for supercharacters is obtained by reversing signs in the numerator of the right-hand side of (7.5).

Letting all $\epsilon_{i}$ and $\Lambda_{0}$ equal 0 in (7.5), we obtain:

$$
t r_{\Phi_{V}^{+}} q^{L_{0}} \pm t r_{\Phi_{V}^{-}} q^{L_{0}}=\Pi_{k=1}^{\infty} \frac{\left(1 \pm q^{k-\frac{1}{2}}\right)^{M}}{\left(1 \mp q^{k-\frac{1}{2}}\right)^{N}} .
$$


Noticing that

$$
\Pi_{k=1}^{\infty}\left(1-q^{k-\frac{1}{2}}\right)=\frac{\varphi\left(q^{\frac{1}{2}}\right)}{\varphi(q)} \text { and } \Pi_{k=1}^{\infty}\left(1+q^{k-\frac{1}{2}}\right)=\frac{\varphi(q)^{2}}{\varphi\left(q^{\frac{1}{2}}\right) \varphi\left(q^{2}\right)}
$$

and using the asymptotics (4.11) of $\eta(\tau)$, we obtain the following asymptotics as $\tau \downarrow 0$ :

$$
\operatorname{tr}_{\Phi_{V}^{ \pm}} q^{L_{0}} \sim \frac{1}{2^{n+1}} e^{\frac{\pi i}{12 \tau}\left(\frac{1}{2} M+N\right)} .
$$

Remark 7.3. The right-hand side of (7.6) multiplied by $q^{(N-M) / 48}$ is a modular function equal to a product of powers of functions $\eta\left(\frac{1}{2} \tau\right) / \eta(\tau)$ and $\eta(\tau)^{2} / \eta\left(\frac{1}{2} \tau\right) \eta(2 \tau)$, and the same holds if we replace $t r$ by $s t r$. It is well known (and easy to see) that the above two modular functions along with the modular function $\eta(2 \tau) / \eta(\tau)$ are transitively permuted (with some constant factors) under the action of $S L(2, \mathbb{Z})$. Thus, the normalized by $q^{(N-M) / 48}$ characters and supercharacters of integrable level one $\operatorname{osp}(M \mid N)$-modules are modular functions, but their $\mathbb{C}$-span is not $S L(2, \mathbb{Z})$-invariant.

\section{On classification of modules over the associated vertex algebras}

Define numbers $u$ and $h^{\vee}$ (the dual Coxeter number) by

$$
\text { level }\left(k u \Lambda_{0}\right)=k \text {, level }(\rho)=h^{\vee} \text {. }
$$

Their values for all affine superalgebras are given in Table 5 .

Table 5:

\begin{tabular}{c|ccccccc}
\hline \hline$\widehat{\mathfrak{g}}$ & $A(m, n)$ & $B(m, n)$ & $C(n)$ & $D(m, n)$ & $D(2,1 ; a)$ & $F(4)$ & $G(3)$ \\
\hline & 1 & $-1 / 2$ & $-1 / 2$ & $-1 / 2$ & $-(a+1)^{-1}$ & $-2 / 3$ & $-3 / 4$ \\
\hline$h^{\vee}$ & $m-n$ & $2(m-n)-1$ & $n-1$ & $2(m-n-1)$ & 0 & 3 & 2
\end{tabular}

The following proposition is an immediate corollary of Theorems 6.1 and 6.2.

Proposition 8.1. (a) If $\widehat{\mathfrak{g}}=A(m, n)$ with $m \geq 1, B(m, n)$ with $m \geq 2, D(m, n)$, $F(4)$ or $G(3)$, then the $\widehat{\mathfrak{g}}$-module $L\left(k u \Lambda_{0}\right)$ is principal integrable iff $k \in \mathbb{Z}_{+}$.

The $B(1, n)$-module $L\left(k u \Lambda_{0}\right)$ is principal integrable iff $k \in \mathbb{Z}_{+} \cup\left\{2 n-\frac{1}{2}+\mathbb{Z}_{+}\right\}$. The $D(2,1 ; a)$-module $L\left(k u \Lambda_{0}\right)$ is principal integrable iff $k \in \mathbb{Z}_{+} \cap a \mathbb{Z}_{+}$.

(b) If $\widehat{\mathfrak{g}}=B(m, n) \hat{)}, C(n) \hat{)}, D(m, n) \widehat{,} D(2,1 ; a), F(4)$ or $G(3)$, then the $\widehat{\mathfrak{g}}$-module $L\left(k_{0} \Lambda_{0}\right)$ is subprincipal integrable iff $k_{0} \in \mathbb{Z}_{+}$.

Recall that the $\widehat{\mathfrak{g}}$-module $V_{k}:=L\left(k u \Lambda_{0}\right)$ has a canonical structure of a vertex algebra for any $k \in \mathbb{C}$ (see e.g. [K4]). It is well known that any irreducible $V_{k}$-module is one of the (irreducible) $\widehat{\mathfrak{g}}$-modules $L(\Lambda)$ of level $k$, and it is an important problem of vertex algebra theory to find out which of these $L(\Lambda)$ are actually $V_{k}$-modules. A necessary condition is given by 
Proposition 8.2. Suppose that $k$ is such that $L\left(k u \Lambda_{0}\right)$ is a principal (resp. subprincipal) integrable $\widehat{\mathfrak{g}}$-module. If a $\widehat{\mathfrak{g}}$-module $L(\Lambda)$ of level $k$ is a $V_{k}$-module, then it must be a principal (resp. subprincipal) integrable.

Proof. Denote by $\widehat{\mathfrak{g}}^{0}$ the subalgebra $\widehat{\mathfrak{g}}_{\overline{0}}^{\prime}$ (resp. $\left.\widehat{\mathfrak{g}}_{\overline{0}}^{\prime \prime}\right)$ of $\widehat{\mathfrak{g}}$ (see $\S$ ). This is an affine Lie algebra. Denote by $V^{0}$ the vertex subalgebra $U\left(\widehat{\mathfrak{g}}^{0}\right) v_{k u \Lambda_{0}}$ of $V_{k}$. Since, by definition, $V^{0}$ is an integrable $\widehat{\mathfrak{g}}^{0}$-module, it follows that it is $\widehat{\mathfrak{g}}^{0}$-irreducible [K3], hence $V^{0}$ is a simple affine vertex algebra of non-negative integral level. But one knows $\mathbb{Z}$ that all irreducible modules over such a vertex algebra are integrable $\widehat{\mathfrak{g}}^{0}$-modules. Using the complete reducibility of $\widehat{\mathfrak{g}}^{0}$-modules [K3], we deduce that any $V$-module, viewed as a $V^{0}$-module, is a direct sum of irreducible integrable $\widehat{\mathfrak{g}}^{0}$-modules, which proves the proposition.

Let $\widehat{\mathfrak{g}}_{+}=\mathbb{C}[t] \otimes_{\mathbb{C}} \mathfrak{g}+\mathbb{C} d$ and consider a 1-dimensional module $\mathbb{C}_{k}(k \in \mathbb{C})$ over $\widehat{\mathfrak{g}}_{+}+\mathbb{C} K$ on which $\widehat{\mathfrak{g}}_{+}$acts trivially and $K=k$. Then $L\left(k u \Lambda_{0}\right)$ is a quotient of the induced $\widehat{\mathfrak{g}}-$ module $\tilde{V}_{k}=U(\widehat{\mathfrak{g}}) \otimes_{U\left(\widehat{\mathfrak{g}}_{+}+\mathbb{C} K\right)} \mathbb{C}_{k}$ by a left ideal $I_{k}$ of $U(\widehat{\mathfrak{g}})$ applied to $1 \otimes 1$. Suppose that $k$ is such that $L\left(k u \Lambda_{0}\right)$ is a principal integrable $\widehat{\mathfrak{g}}$-module. As we have seen in the proof of Proposition 8.2, viewed as a $\widehat{\mathfrak{g}}_{0}^{\prime}$-module, $L\left(k u \Lambda_{0}\right)$ is a direct sum of irreducible integrable highest weight modules. All these modules have the same level $\ell$ (resp. $\ell_{+}, \ell_{-}$when $\mathfrak{g}_{0}^{\prime}$ has two simple components) given in terms of $k$ as follows:

$$
\begin{aligned}
\ell & =\ell_{+}=k \text { if } \mathfrak{g} \neq B(1, n), \quad \ell=2 k \text { if } \mathfrak{g}=B(1, n) \\
\ell_{-} & =k \text { if } \mathfrak{g}=D(2, n), \quad \ell_{-}=a^{-1} k \text { if } \mathfrak{g}=D(2,1 ; a)
\end{aligned}
$$

In particular, $I_{k}$ contains the element

$$
e_{-\theta^{\prime}}(1)^{\ell+1}\left(\text { resp. elements } e_{-\theta_{+}^{\prime}}(1)^{\ell_{+}^{\prime}+1} \text { and } e_{-\theta_{-}^{\prime}}(1)^{\ell_{-}^{\prime}+1}\right) \text {. }
$$

If elements (8.2) generate the left ideal $I_{k}$, it follows that a $\widehat{\mathfrak{g}}$-module $L(\Lambda)$ of level $k$ is a $V_{k^{-}}$ module iff the field $e_{-\theta^{\prime}}(z)^{\ell+1}$ (resp. fields $e_{-\theta_{+}^{\prime}}^{\ell_{+}^{\prime}+1}(z)$ and $\left.e_{-\theta_{-}^{\prime}}^{\ell^{\prime}+1}(z)\right)$ annihilate $L(\Lambda)$. The latter property implies that, viewed as a $\widehat{\mathfrak{g}}_{0}^{\prime}$-module, $L(\Lambda)$ is a direct sum of irreducible integrable modules and therefore $L(\Lambda)$ is a principal integrable $\widehat{\mathfrak{g}}$-module. We thus established a sufficient condition for a $\widehat{\mathfrak{g}}$-module $L(\Lambda)$ to be a $V_{k}$-module:

Proposition 8.3. Let $k$ be such that $L\left(k u \Lambda_{0}\right)$ is a principal integrable $\widehat{\mathfrak{g}}$-module and suppose that the left ideal $I_{k}$ is generated by (8.2). Let $L(\Lambda)$ be a principal integrable $\widehat{\mathfrak{g}}$-module of level $k$. Then $L(\Lambda)$ is a $V_{k}$-module.

Proposition 8.4. Let $k$ be such that $L\left(k u \Lambda_{0}\right)$ is a principal integrable $\widehat{\mathfrak{g}}$-module.

(a) Suppose that the highest weight $k u \Lambda_{0}$ is the only singular weight of the $\widehat{\mathfrak{g}}$-module $\tilde{V}_{k}$ which is principal integrable. Then elements (8.9) generate the left ideal $I_{k}$.

(b) The assumption of (a) holds if

$$
k+h^{\vee} \neq 0,
$$


and for any principal integrable weight $\Lambda$ of level $k$ one has:

$$
\Lambda-k u \Lambda_{0} \notin \widehat{Q} \backslash \mathbb{Z} \delta,
$$

where $\widehat{Q}=\sum_{i \in \widehat{I}} \mathbb{Z} \alpha_{i}$ is the root lattice of $\widehat{\mathfrak{g}}$.

Proof. Let $I_{k}^{\prime}\left(\subset I_{k}\right)$ denote the left ideal of $U(\widehat{\mathfrak{g}})$ generated by elements (8.2). Then the $\widehat{\mathfrak{g}}$ module $V_{k}^{\prime}=\tilde{V}_{k} /\left(I_{k}^{\prime}(1 \otimes 1)\right)$ is principal integrable, hence each of its singular weights $\Lambda$ is integrable. Hence, if the condition of (a) holds, the $\widehat{\mathfrak{g}}$-module $V_{k}^{\prime}$ is irreducible, and therefore $I_{k}^{\prime}=I_{k}$.

Furthermore, obviously, $\Lambda-k u \Lambda_{0} \in \widehat{Q}$, hence (8.4) implies that $\Lambda=k u \Lambda_{0}+j \delta$ for some $j \in \mathbb{Z}$. Using the Casimir operator [K3], we obtain:

$$
\left(k u \Lambda_{0}+\rho \mid k u \Lambda_{0}+\rho\right)=\left(k u \Lambda_{0}+\rho-j \delta \mid k u \Lambda_{0}+\rho-j \delta\right),
$$

which is equivalent to $j\left(k+h^{\vee}\right)=0$. But then (8.3) implies that $j=0$, proving (b).

Theorem 8.1. Let $\widehat{\mathfrak{g}}$ be one of the affine superalgebras $A(m, n)$ with $m \geq 1, B(m, n)$ with $m \geq 1, D(m, n) \hat{)}, D(2,1 ; a)$ with $a^{-1} \in \mathbb{N}, F(4)$ or $G(3)$. Then all integrable $\widehat{\mathfrak{g}}$-modules $L(\Lambda)$ of level 1 are $V_{1}$-modules (the complete list of these $\Lambda$ 's is given by Remark 6.3).

Proof. Note that in the $A(m, n)^{\wedge}$ case $V_{1}$ is a subalgebra of the vertex subalgebra $F_{0}$ of $F$ (constructed in $\S$ 3), while the highest component of the $F_{0}$-module $F_{s}$ restricted to $V_{1}$ is $L\left(\Lambda_{(s)}\right)$. Since the $\Lambda_{(s)}$ exhaust all integrable highest weights of level 1, by Proposition 8.1, they give a complete list of irreducible $V_{1}$-modules.

In the $B(m, n)$ and $D(m, n)$ cases we note that $V_{1}$ is isomorphic to the vertex algebra $\Phi_{V}^{+}$ (see Theorem 7.1), $\Phi_{V}^{-}$is its irreducible module, and these two modules produce all integrable highest weights of level 1.

The cases $F(4)$ and $G(3)$ are obvious since $V_{1}$ is the only irreducible integrable module of level 1 (see Remark 6.3).

It remains to show that $L\left(-\frac{a+2}{a+1} \Lambda_{0}-\Lambda_{1}+\frac{1-a}{a} \Lambda_{3}\right)$ is a $V_{1}$-module in the $D(2,1 ; a)$ case. But $-\frac{a+2}{a+1} \Lambda_{0}-\Lambda_{1}+\frac{1-a}{a} \Lambda_{3}=-\frac{1}{a+1} \Lambda_{0}-\left(\frac{1}{2} \alpha_{0}+\frac{a-1}{2 a} \alpha_{3}\right)$, hence the difference of this weight and $u \Lambda_{0}$ does not lie in the root lattice; we also have: $k=1$ and level $h^{\vee}=0$. Hence we may apply Propositions 8.4 and 8.3 .

Remark 8.1. The lowest energy $D(2,1 ; a)$-submodule of the module $L\left(-\frac{a+2}{a+1} \Lambda_{0}-\Lambda_{1}+\frac{1-a}{a} \Lambda_{3}\right)$ is the module $\bar{L}\left(-\bar{\Lambda}_{1}+\left(a^{-1}-1\right) \bar{\Lambda}_{3}\right)$. It has dimension $4 a^{-1}+2$. For $a=1$ this is the defining module of $D(2,1)$; for $a=\frac{1}{2}$ (resp. $\frac{1}{3}$ ) this is the 10- (resp. 14-) dimensional module mentioned in Remark 6.8. As a $D(2,1 ; a)$-module, the even (resp. odd) part of this module is isomorphic to the irreducible $s \ell(2)+s \ell(2)+s \ell(2)$-module

$$
\mathbb{C} \otimes \mathbb{C}^{a^{-1}} \otimes \mathbb{C}^{2}\left(\text { resp. } \mathbb{C}^{2} \otimes \mathbb{C}^{a^{-1}+1} \otimes \mathbb{C}\right)
$$


Remark 8.2. Let $V$ be a vertex algebra with a conformal vector such that $L_{0}$ is diagonizable with finite-dimensional eigenspaces and rational eigenvalues. It is a general belief that if $V$ has finitely many irreducible modules, then the character $\operatorname{tr}_{M} q^{L_{0}}$ of each of these modules $M$ becomes a modular function when normalized, i.e., multiplied by a suitable power of $q$. The example of the vertex algebra $V_{1}$ for $B(m, n)$ and $D(m, n)$ confirms this conjecture and leads to believe that the same is true for $D(2,1 ; a)$ (with $\left.a^{-1} \in \mathbb{N}\right), F(4)$ and $G(3)$.

The vertex algebra $V$ is called rational if $L_{0}$ has integral eigenvalues, the number of irreducible $V$-modules is finite and any $V$-module is completely reducible. It follows from the above discussion that the vertex algebra $V_{1}$ for $\left.B(m, n) \hat{)}, D(m, n) \widetilde{f} D(2,1 ; a)\right), F(4) \hat{)}$ and $G(3)$ is a rational vertex algebra, and that, moreover, the corresponding Zhu algebra [Z] is finitedimensional semisimple (and even 1-dimensional in the $F(4)^{\Upsilon}$ and $G(3)^{-}$cases).

It was proved by Zhu [B] under certain technical assumptions that the $\mathbb{C}$-span of normalized characters of irreducible modules over a rational vertex algebra is $S L(2, \mathbb{Z})$-invariant, and it was believed by many that the technical assumptions may be removed. However, the above mentioned rational vertex algebra $V_{1}$ shows that this is not the case.

Remark 8.3. There are only two cases where there exists only a finite number of essentially inequivalent subprincipal integrable $\widehat{\mathfrak{g}}$-modules of a given non-zero level $k$ :

$$
\widehat{\mathfrak{g}}=F(4), k=-\frac{3}{2} \text { and } \widehat{\mathfrak{g}}=G(3) \widehat{,}, k=-\frac{4}{3} .
$$

In both cases the only subprincipal integrable $\widehat{\mathfrak{g}}$-module is $L\left(\Lambda_{0}\right)$. In both cases the associated vertex algebra is rational with a unique irreducible module and the Zhu algebra is 1-dimensional.

\section{$9 \quad$ Some remarks and open problems}

9.1 The calculation of characters of integrable highest weight modules of arbitrary level $k$ over affine superalgebras seems to be a very difficult problem. One may expect that the case of the "critical" level $k=-h^{\vee}$ should be rather different from other levels (as for the affine Lie algebras). However, the construction of level 1 integrable modules over $\operatorname{osp}(m \mid n)$ given in $\S$ Q is the same for all values $m$ and $n$ though 1 is the critical level iff $m-n=1$.

Formula (5.12) leads us to believe in the following conjecture:

Consider a principal integrable highest weight module $L(\Lambda)$ over an affine superalgebra $\widehat{\mathfrak{g}}$ and suppose that one can choose a set of simple roots $\widehat{\Pi}$ such that it contains a maximal $\Lambda+\rho$ isotropic subset $S_{\Lambda}$ of roots (i.e., all roots from $S_{\Lambda}$ are pairwise orthogonal and orthogonal to $\Lambda+\rho[\overline{\mathrm{KW}}]$ ). Let $\widehat{W}^{\#}$ be the Weyl group of the integrable part $\widehat{\mathfrak{g}}_{\overline{0}}^{\prime}$ of $\widehat{\mathfrak{g}}_{\overline{0}}$. We conjecture that the following character formula holds:

$$
e^{\rho} \operatorname{Rch} L(\Lambda)=\sum_{w \in \widehat{W}^{\#}} \epsilon(w) w \frac{e^{\Lambda+\rho}}{\Pi_{\beta \in S_{\Lambda}}\left(1+e^{-\beta}\right)} .
$$

Note that the assumptions of this conjecture exclude the critical level, and include the level 1 integrable modules over exceptional affine superalgebras.

9.2 In the papers [KW1] and [KW2 we proved character formulas for a class of modules $L(\Lambda)$ over affine Lie algebras, called admissible modules, which includes integrable modules. These 
character formulas imply that the normalized specialized characters of admissible modules are modular functions (and we conjecture that this property characterizes admissible modules). Of course, these character formulas break down in the Lie superalgebra case. However, in certain exceptional situations, when the character admits a simple product expansion in the Lie algebra case (see KW2], Theorem 3.2), it seems that a similar product formula holds in the Lie superalgebra case as well.

Concretely, let $u$ be a positive integer and let

$$
k=h^{\vee}\left(u^{-1}-1\right)
$$

(recall that in general the level $k$ of an admissible module is $\geq h^{\vee}\left(u^{-1}-1\right)$ ). Let $y$ be an automorphism of the root lattice $\widehat{Q}$ such that all roots $\gamma_{i}=y\left((u-1) \delta_{i 0} K+\alpha_{i}^{\vee}\right)$ are positive $(i \in \widehat{I})$. The weights of the form $y \cdot k \Lambda_{0}$, where, as usual, $y \cdot \lambda=y(\lambda+\rho)-\rho$, are called admissible. We conjecture that the following analog of formula (3.3) from [KW2 holds:

$$
\operatorname{ch} L\left(y \cdot\left(k \Lambda_{0}\right)\right)=e^{y \cdot\left(k \Lambda_{0}\right)}\left(\frac{\varphi\left(q^{u}\right)}{\varphi(q)}\right)^{\ell} \prod_{\substack{\alpha \in \bar{\Delta}_{\overline{0}} \\ n \in \mathbb{N}}} \frac{1-q^{u n} e^{y \cdot \alpha}}{1-q^{n} e^{\alpha}} / \Pi_{\substack{\alpha \in \bar{\Delta}_{\overline{1}} \\ n \in \mathbb{N}}} \frac{1+q^{u n} e^{y \cdot \alpha}}{1+q^{n} e^{\alpha}},
$$

where $\ell$ is the rank of $\mathfrak{g}, \bar{\Delta}_{\overline{0}}, \bar{\Delta}_{\overline{1}}$ are the sets of even and odd roots of $\mathfrak{g}$, and $q=e^{-\delta}$.

This conjecture agrees with formula (7.5) in the case $\widehat{\mathfrak{g}}=o s p(2 \mid 2)=s \ell(2 \mid 1) \hat{)}$. In this case $k=-1 / 2$ and $h^{\vee}=1$, so that (9.2) holds for $u=2$. All the admissible weights of level $-\frac{1}{2}$ are as follows:

$$
-\frac{1}{2} \Lambda_{i}(i=0,1,2),-\frac{1}{2} \Lambda_{0}-\frac{1}{2} \alpha_{0}
$$

where the Dynkin diagram is chosen such that $\alpha_{1}$ and $\alpha_{2}$ are odd roots (and $\alpha_{0}$ is even). Character formula (7.5) gives:

$$
\begin{gathered}
\operatorname{ch}\left(-\frac{1}{2} \Lambda_{0}\right) \\
\operatorname{ch}\left(-\frac{1}{2} \Lambda_{0}-\frac{1}{2} \alpha_{0}\right)
\end{gathered}=\frac{1}{2} e^{-\frac{1}{2} \Lambda_{0}}\left(\frac{\Psi\left(u^{-1} v q^{\frac{1}{2}} ; q\right)}{\Psi\left(-u v q^{\frac{1}{2}} ; q\right)} \pm \frac{\Psi\left(-u^{-1} v q^{\frac{1}{2}} ; q\right)}{\Psi\left(u v q^{\frac{1}{2}} ; q\right)}\right),
$$

where $u=e^{-\frac{1}{2} \alpha_{1}}, v=e^{-\frac{1}{2} \alpha_{2}}$ and

$$
\Psi(z ; q)=\Pi_{k=1}^{\infty}\left(1+z q^{k-1}\right)\left(1+z^{-1} q^{k}\right),
$$

whereas formula $(9.3)$ gives:

$$
\begin{aligned}
\operatorname{ch}\left(-\frac{1}{2} \Lambda_{0}\right) & =e^{-\frac{1}{2} \Lambda_{0}} \frac{\Psi\left(u^{2} q ; q^{2}\right) \Psi\left(v^{2} q ; q^{2}\right) \varphi\left(q^{2}\right)^{2}}{\Psi\left(-u v q ; q^{2}\right) \varphi(q)^{2}} \\
\operatorname{ch}\left(-\frac{1}{2} \Lambda_{0}-\frac{1}{2} \alpha_{0}\right) & =e^{-\frac{1}{2} \Lambda_{0}-\frac{1}{2} \alpha_{0}} \frac{\Psi\left(u^{2} ; q^{2}\right) \Psi\left(v^{2} ; q^{2}\right) \varphi\left(q^{2}\right)^{2}}{\Psi\left(-u v q ; q^{2}\right) \varphi(q)^{2}} .
\end{aligned}
$$

However, the seemingly different expressions in the right-hand sides of (9.4) and (9.6) actually coincide due to one of the addition theta function formulas (cf. [M], formula (6.6) and notation on p. 17):

$$
\theta_{00}\left(\tau, z_{1}\right) \theta_{00}\left(\tau, z_{2}\right)=\theta_{00}\left(2 \tau, z_{1}+z_{2}\right) \theta_{00}\left(2 \tau, z_{1}-z_{2}\right)+\theta_{10}\left(2 \tau, z_{1}+z_{2}\right) \theta_{10}\left(2 \tau, z_{1}-z_{2}\right)
$$


if we let $u=e^{2 \pi i z_{1}}, v=e^{2 \pi i z_{2}}$.

Using [KW], formula (6.1), it is immediate to show that the span of supercharacters of the four admissible $s \ell(2 \mid 1)$-modules of level $-1 / 2$ is $S L(2, \mathbb{Z})$-invariant. Thus, it is natural to conjecture that this modular invariance property of admissible characters holds for any affine superalgebra $\widehat{\mathfrak{g}}$ and any $k$ given by $(9.2)$.

Two other very interesting examples are provided by Remark 8.3: $\widehat{\mathfrak{g}}=F(4)$ with $u=2$, $y=1$ and $\widehat{\mathfrak{g}}=G(3)^{\widehat{w}}$ with $u=3, y=1$.

9.3 The first case not covered in $\S$, that when $m=n-1$, is very interesting. It connects the level 1 modules over $\mathfrak{g} \ell(n-1 \mid n)$ (or, equivalently the "critical" level -1 modules over $g \ell(n \mid n-1)$ ) to the denominator identity for $s \ell(n \mid n)$, which is unknown. Analyzing this connection, we arrived at the following $s \ell(2 \mid 2)$ denominator identity:

$$
e^{\rho} R=\sum_{w \in \widehat{W}^{\#}} \epsilon(w) w \frac{e^{\rho}}{\left(1+e^{\alpha_{0}}\right) \Pi_{j=1}^{\infty}\left(1+q^{j} e^{\alpha_{2}}\right)\left(1+q^{j-1} e^{-\alpha_{2}}\right)}
$$

where, as before, $q=e^{-\delta}$. Here we use the Dynkin diagram with the grey nodes $\alpha_{0}$ and $\alpha_{2}$. If all four nodes are grey we get the same identity with $\rho$ replaced by 0 ; in yet another form (9.8) can be written as follows:

$$
\begin{array}{r}
\frac{R}{\varphi(q)} \Pi_{n=1}^{\infty}\left(1-q^{2 n}\right)\left(1+q^{2 n-1} e^{\alpha_{1}+\alpha_{3}}\right)\left(1+q^{2 n-1} e^{-\alpha_{1}-\alpha_{3}}\right) \\
=\sum_{w \in \widehat{W}^{\#}} \epsilon(w) w\left(\Pi_{n=1}^{\infty}\left(1+q^{n} e^{\alpha_{1}}\right)\left(1+q^{n} e^{\alpha_{3}}\right)\left(1+q^{n-1} e^{-\alpha_{1}}\right)\left(1+q^{n-1} e^{-\alpha_{3}}\right)\right) .
\end{array}
$$

The latter identity is equivalent to the following identity in $u=e^{-\alpha_{1}}, x=e^{-\alpha_{2}}, v=e^{-\alpha_{3}}$ and $q$ (where $\Psi$ is defined by $(9.5))$ :

$$
\begin{aligned}
& \Psi\left(u v q ; q^{2}\right) \Psi(-u x ; q) \Psi(-v x ; q) \\
& \quad=\Psi\left(u v^{-1} q ; q^{2}\right) \Psi(x ; q) \Psi(u v x ; q)-x \Psi\left(u v x^{2} q ; q^{2}\right) \Psi(u ; q) \Psi(v ; q) .
\end{aligned}
$$

In the notation of [M] this identity can be rewritten in terms of theta functions as follows (if we let $\left.u=e^{2 \pi i z_{1}}, v=e^{2 \pi i z_{2}}, x=e^{2 \pi i z_{3}}\right)$ :

$$
\begin{aligned}
& \theta_{00}\left(2 \tau, z_{1}+z_{2}\right) \theta_{11}\left(\tau, z_{1}+z_{3}\right) \theta_{11}\left(\tau, z_{2}+z_{3}\right) \\
+ & \theta_{00}\left(2 \tau, z_{1}-z_{2}\right) \theta_{10}\left(\tau, z_{3}\right) \theta_{11}\left(\tau, z_{1}+z_{2}+z_{3}\right) \\
= & \theta_{00}\left(2 \tau+z_{1}+z_{2}+2 z_{3}\right) \theta_{10}\left(\tau, z_{1}\right) \theta_{10}\left(\tau, z_{2}\right) .
\end{aligned}
$$

Identity (9.10) can be derived from (9.7) as follows. Replacing $z_{i}$ by $z_{i}+\frac{1}{2} \tau$ (resp. $z_{i}+\frac{1}{2}(1+\tau)$ ) in (9.7), we obtain:

$$
\begin{aligned}
& \theta_{10}\left(\tau, z_{1}\right) \theta_{10}\left(\tau, z_{2}\right)=\theta_{00}\left(2 \tau, z_{1}+z_{2}\right) \theta_{10}\left(2 \tau, z_{1}-z_{2}\right)+\theta_{10}\left(2 \tau, z_{1}+z_{2}\right) \theta_{00}\left(2 \tau, z_{1}-z_{2}\right) \\
& \theta_{11}\left(\tau, z_{1}\right) \theta_{11}\left(\tau, z_{2}\right)=\theta_{00}\left(2 \tau, z_{1}+z_{2}\right) \theta_{10}\left(2 \tau, z_{1}-z_{2}\right)-\theta_{10}\left(2 \tau, z_{1}+z_{2}\right) \theta_{00}\left(2 \tau, z_{1}-z_{2}\right)
\end{aligned}
$$


Substituting 9.11b) (resp. (9.11a)) in the first (resp. second) summand of the left-hand side of (9.10), we obtain the product of $\theta_{00}\left(2 \tau, z_{1}+z_{2}+2 z_{3}\right)$ and the right-hand side of (9.11a), and, substituting its left-hand side, we obtain the left-hand side of (9.10).

We also have a conjectural formula for an $s \ell(3 \mid 3)$ denominator identity, but it is too cumbersome to be reproduced here. We have no conjectures as how the $s \ell(n \mid n)$ denominator identity should look for $n>3$.

Using the connection of the $s \ell(2 \mid 2)^{\Upsilon}$ denominator identity to level 1 modules over $s \ell(2 \mid 1)$ we deduce from (9.8) $\left(k \in \mathbb{Z}_{+}\right)$:

$$
\text { (9.12) } \operatorname{chL}\left(k \Lambda_{0}-(k+1) \Lambda_{1}\right)=\frac{\varphi(q)}{e^{\rho} R} \sum_{w \in\left\langle r_{0}\right\rangle} \epsilon(w) w \sum_{j \in \mathbb{Z}_{+}} t_{j \alpha_{0}} \frac{e^{\Lambda+\rho}}{\Pi_{n=1}^{\infty}\left(1+q^{n-1} e^{-\alpha_{2}}\right)\left(1+q^{n} e^{\alpha_{2}}\right)} .
$$

Here the Dynkin diagram is chosen in such a way that $\alpha_{0}$ is even and $\alpha_{1}, \alpha_{2}$ are odd simple roots.

9.4 Let $k$ be such that $V_{k}=L\left(u k \Lambda_{0}\right)$ is a (principal or subprincipal) integrable $\widehat{\mathfrak{g}}$-module of level $k$. Is it always true that any integrable $\widehat{\mathfrak{g}}$-module of level $k$ can be extended to a module over the vertex algebra $V_{k}$ ? Of course, this question is closely related to the description of generators of the left ideal $I_{k}$. In the principal integrable case $I_{k}$ contains elements (8.2), and the answer to the above question in this case would be positive if $I_{k}$ were generated by these elements.

Is it true that the normalized (by a power of $q$ ) characters $\operatorname{trq}^{L_{0}}$ (where $L_{0}$ is given by the Sugawara construction [K4]) of irreducible $V_{k}$-modules are modular functions, provided that there are finitely many of them and $k+h^{\vee} \neq 0$. Is it true that for $k$ of the form (9.2), all $V_{k}$-modules are admissible modules?

9.5 A few examples that we have worked out in the paper indicate that the theory of integrable highest weight modules over affine Lie superalgebras is dramatically different from that in the Lie algebra case. The only exception is the case of $\widehat{\mathfrak{g}}=B(0, n) \widehat{\text {. The integrability conditions }}$ are (see Table 11 for its Dynkin diagram).

$$
k_{i} \in \mathbb{Z}_{+} \text {for all } i, \quad k_{n} \in 2 \mathbb{Z}_{+},
$$

hence the level $k=2 k_{0}+\ldots+2 k_{n-1}+k_{n}$ is a non-negative even integer and the number of integrable highest weight $B(0, n)$-modules is finite for each $k$. Moreover, each of these modules extends to an irreducible $V_{k}$-module since $I_{k}$ is generated by $e_{-\theta}(1)^{k+1}$ for each $k$, and these are all irreducible modules over the vertex algebra $V_{k}\left(k \in 2 \mathbb{Z}_{+}\right)$.

Furthermore, the character formula for all integrable $B(0, n)$-modules $L(\Lambda)$ is known (see [K2]), and it is given by the same expression as that for the twisted affine algebra $A_{2 n}^{(2)}$ (replacing the black node by a white one). In order to derive the transformation formula of $B(0, n)$ supercharacters from that of $A_{2 n}^{(2)}$ characters, we need to go from the $A_{2 n}^{(2)}$ coordinates, which we call $A$-coordinates, to the $B(0, n)^{\wedge}$ coordinates, which we will call $B$-coordinates. This calculation is explained below.

The $B$-coordinates $\left(\tau, z_{B}, u_{B}\right)$ of $h \in \widehat{\mathfrak{h}}$ are defined by

$$
h=2 \pi i\left(-\tau \frac{1}{2} \Lambda_{0}+z_{B}+u_{B} \delta\right), \text { where } z_{B} \in \mathfrak{h} .
$$


Let $\beta=\frac{1}{2} \sum_{j=0}^{n-1}(n-j) \alpha_{j}^{\vee}$. Then $t_{\beta}\left(\Lambda_{i}\right)=\Lambda_{i}$ for $i=1, \ldots, n-1$ and $\left\langle t_{-\beta}\left(\frac{1}{2} \Lambda_{0}\right), \alpha_{i}^{\vee}\right\rangle=\delta_{0 n}$, hence we may take $\Lambda_{n}=t_{-\beta}\left(\frac{1}{2} \Lambda_{0}\right)$ for the $0^{\text {th }}$ fundamental weight of $A_{2 n}^{(2)}$. Hence the $A$-coordinates are expressed via $B$-coordinates by

$$
\tilde{h}=t_{-\beta}(h)=2 \pi i\left(-\tau \Lambda_{n}+z_{A}+u_{A} \delta\right) .
$$

Recall that $S L(2, \mathbb{Z})$ acts on functions in $\tau, z, u$ by the formula [K3, Chapter 13]:

$$
\left.F(\tau, z, u)\right|_{\left(\begin{array}{ll}
a & b \\
c & d
\end{array}\right)}=j(\tau)^{-n} F\left(\frac{a \tau+b}{c \tau+d}, \frac{z}{c \tau+d}, u-\frac{c(z \mid z)}{2(c \tau+d)}\right) .
$$

Furthermore, defining a new function $F^{\alpha, \beta}$ by

$$
F^{\alpha, \beta}(h)=F\left(t_{\beta}(h)+2 \pi i \alpha-\pi i(\alpha \mid \beta) \delta\right),
$$

we have [KP2]:

$$
\left.F^{\alpha, \beta}\right|_{\left(\begin{array}{ll}
a & b \\
c & d
\end{array}\right)}=\left(\left.F\right|_{\left(\begin{array}{ll}
a & b \\
c & d
\end{array}\right)}\right)^{d \alpha-b \beta, a \beta-c \alpha} .
$$

We shall use the following connection between supercharacters of $B(0, n)$ and characters of $A_{2 n}^{(2)}$, which follows from (9.13) and definitions:

$$
\operatorname{sch} L(\Lambda)(h)=\operatorname{chL}(\Lambda)(\tilde{h})^{-\beta, \beta}=(-1)^{n k / 2} \operatorname{ch} L(\Lambda)(\tilde{h})^{\beta, \beta} .
$$

Recall that the normalized $A_{2 n}^{(2)}$ character $\tilde{\chi}_{\Lambda}$ and the normalized $B(0, n)$ supercharacter $\chi_{\Lambda}$ are defined by:

$$
\tilde{\chi}_{\Lambda}=q^{\tilde{m}_{\Lambda}} \operatorname{ch} L(\Lambda), \chi_{\Lambda}=q^{m_{\Lambda}} \operatorname{sch} L(\Lambda),
$$

where in $B$-coordinates:

$$
m_{\Lambda}=\frac{|\Lambda+\rho|^{2}}{2\left(k+h^{\vee}\right)}-\frac{|\rho|^{2}}{2 h^{\vee}}=\frac{(\Lambda+2 \rho \mid \Lambda)}{2\left(k+h^{\vee}\right)}-\frac{c_{k}}{24}, \quad c_{k}=\frac{k \operatorname{sdim} B(0, n)}{k+h^{\vee}},
$$

and $\tilde{m}_{\Lambda}$ is defined by a similar formula in $A$-coordinates. (Hence $\chi_{\Lambda}(\tau, 0,0)=\operatorname{str}_{L(\Lambda)} q^{L_{0}-c_{k} / 24}$, as it should be.)

Let $S=\left(\begin{array}{cc}0 & -1 \\ 1 & 0\end{array}\right) \in S L(2, \mathbb{Z})$. We shall denote by $S_{A}$ (resp. $\left.S_{B}\right)$ the action of $S$ in $A$ (resp. $B$-coordinates). We have by (9.15):

$$
\left.\chi_{\Lambda}\right|_{S_{B}}=\tilde{\chi}_{\Lambda}^{-\beta, \beta}=\left(\left.\tilde{\chi}_{\Lambda}\right|_{S_{A}}\right)^{\beta, \beta},
$$

where the last equality holds due to (9.14).

But one has (see [KP2], K3], Theorem 13.8a):

$$
\left.\tilde{\chi}_{\Lambda}\right|_{S_{A}}=\sum_{M \in P_{+}^{k} \bmod \mathbb{C} \delta} S_{\Lambda M} \tilde{\chi}_{M},
$$


where $\left(S_{\Lambda M}\right)$ is an explicitly known matrix. Hence, continuing the calculation (9.16), we get, using (9.15):

$$
\left.\chi_{\Lambda}\right|_{S_{B}}=\sum_{M \in P_{+}^{k} \bmod \mathbb{C} \delta} S_{\Lambda M} \tilde{\chi}_{M}^{\beta, \beta}=(-1)^{n k / 2} \sum_{M} S_{\Lambda M} \tilde{\chi}_{M}^{-\beta, \beta} .
$$

Using again (9.15), we obtain the final transformation formula:

$$
\left.\chi_{\Lambda}\right|_{S_{B}}=(-1)^{n k / 2} \sum_{M \in P_{+}^{k} \bmod \mathbb{C} \delta} S_{\Lambda M} \chi_{M}
$$

It is clear from the above calculation that the $S L(2, \mathbb{Z})$-invariance of normalized $B(0, n)$ characters $\tilde{\chi}_{\Lambda}$ does not hold, but the span of $\left\{\tilde{\chi}_{\Lambda}, \tilde{\chi}_{\Lambda}^{\beta, 0}, \tilde{\chi}_{\Lambda}^{0, \beta}\right\}_{\Lambda \in P_{+}^{k} \bmod \mathbb{C} \delta}$ is $S L(2, \mathbb{Z})$-invariant.

9.6 We use this opportunity to make some corrections to [KW].

Due to computer error the following lines disappeared from the paper:

(1) Bottom of page 418:

and $4(n+1)^{2}$, respectively (given by Theorem 4.2 ; see also Examples 5.3 and

(2) Bottom of page 421:

(this is independent of the choice of $B$ ), and let $W^{\#}$ denote the subgroup of $W$ generated by reflections $r_{\alpha}$ with respect to all $\alpha \in \Delta_{0}^{\#}$. Denote by (.|.) the even

Also, the diagrams $B(0, n), D(m, n)$ and $D(2,1 ; \alpha)$ on p. 429 should be as follows:

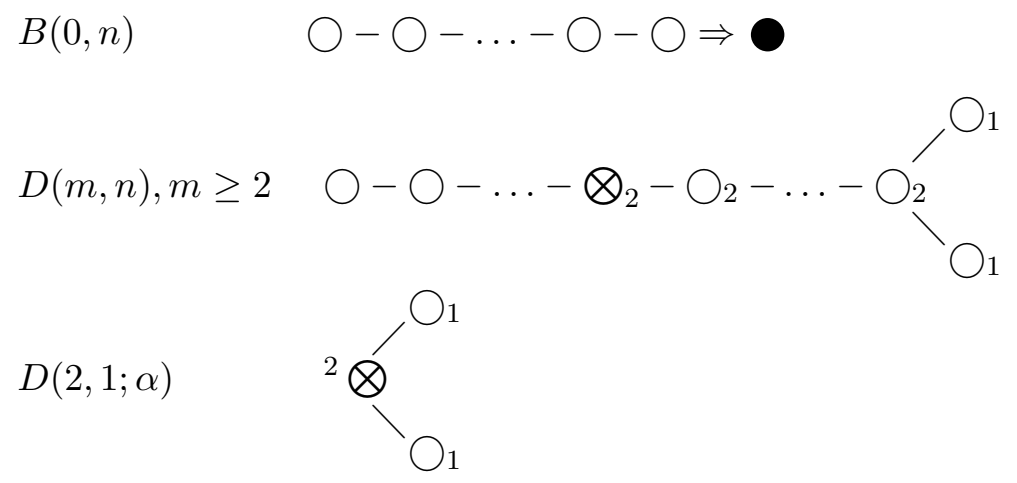

Furthermore, the following corrections should be made:

page 417 , line $13 \uparrow: \square(-q)$

page 432 , line $12 \downarrow: M_{\Lambda}:=\left\{\alpha \in \bar{\Delta}_{0} \mid \alpha \perp S_{\Lambda}\right\}$,

page 434 , lines $5,7 \downarrow: \alpha_{2}$ should be replaced by $\alpha_{1}$,

page 435 , line $6 \downarrow:\left(\bigoplus_{j>0} t^{j} \otimes \mathfrak{g}\right)$, 
page 438 , line $1 \uparrow::\left(\alpha_{1} \mid \alpha_{1}\right)=0$,

page 449 , line $3 \uparrow:\left(\alpha_{2} \mid \alpha_{2}\right)=2$,

page 450 , line $4 \uparrow: \widehat{R}=R_{m} \Pi_{n=1}^{\infty} \ldots$, where $R_{m}$ is the denominator of $A_{m}$, not the one defined by (7.1),

page 453: Theorem 8.1(a) as stated holds for the subprincipal integrable modules (cf. Theorem 6.2 of the present paper).

It is appropriate to mention here that the specialization (7.2) of Conjecture 7.2 has been proved recently independently by S. Milne (by combinatorial methods) and by D. Zagier (using cusp forms). D. Zagier also proved Conjecture 7.2 in the first unknown case $\mathrm{m}=2$.

In a slightly different form than in [KW], Conjecture 7.2 reads:

$$
\begin{aligned}
\Pi_{n=1}^{\infty}\left(\left(\frac{1-q^{2 n}}{1-q^{2 n-1}}\right)^{2 s} \Pi_{\alpha \in \Delta} \frac{1-q^{2 n} e^{\alpha}}{1-q^{2 n-1} e^{\alpha}}\right)= & \sum_{\substack{n_{1}, \ldots, n_{s} \geq 0 \\
k_{1} \geq \ldots \geq k_{s} \geq 0}} \operatorname{ch} L\left(\sum_{i=1}^{s} k_{i} \gamma_{i}, A_{m}\right) \\
& \times q^{\sum_{i=1}^{s} k_{i}\left(2 n_{i}+1\right)+(m-2 i+2) n_{i}} .
\end{aligned}
$$

Here $\Delta$ is the set of roots of $A_{m}, s=\left[\frac{m+1}{2}\right]$ and $\left\{\gamma_{1}, \ldots, \gamma_{s}\right\}$ is the set of positive pairwise orthogonal roots, $\gamma_{1}$ being the highest root.

\section{References}

[A] M.P. Appell, "Sur le fonctions doublement periodique de troisieme espese", Annals Sci. l'Ecole Norm. Sup., 3e serie, t1, p135, t2, p9, t3, p9 (1884-1886).

[B] R. Borcherds, "Monstrous moonshine and monstrous Lie superalgebras", Invent. Math., 109 (1992), 405-444.

[F] I.B. Frenkel, "Two constructions of affine Lie algebra representations and bosonfermion correspondence in quantum field theory", J. Funct. Anal. 44 (1981), 259-327.

[FF] A.J. Feingold and I.B. Frenkel, "Classical affine algebras", Adv. Math., 56 (1985), $117-172$.

[K1] V.G. Kac, "Lie superalgebras", Adv. Math., 26 (1977), 8-96.

[K2] V.G. Kac, "Infinite-dimensional algebras', Dedekind's $\eta$-function, ...", Adv. in Math., 30 (1978), 85-136.

[K3] V.G. Kac, Infinite dimensional Lie algebras, 3rd edition, Cambridge University Press, 1990.

[K4] V.G. Kac, Vertex algebras for beginners, University lecture series, Vol. 10, AMS, Providence RI, 1996. Second edition, 1998. 
[KL] V.G. Kac and J.W. van de Leur, "Super boson-fermion correspondence", Ann. de l'Institute Fourier 37 (1987), 99-137.

[KP1] V.G. Kac and D.H. Peterson, "Spin and wedge representations of infinite-dimensional Lie algebras and groups", Proc. Natl. Acad. Sci. USA 78 (1981), 3308-3312.

[KP2] V.G. Kac and D.H. Peterson, "Infinite-dimensional Lie algebras, theta functions and modular forms", Adv. Math. 53 (1984), 125-264.

[KW1] V.G. Kac and M. Wakimoto, "Modular invariant representations of infinitedimensional Lie algebras and superalgebras", Proc.Natl.Acad.Sci. USA 85 (1988), 4956-4960.

[KW2] V.G. Kac and M. Wakimoto, "Classification of modular invariant representations of affine algebras", Advanced Ser. Math. Phys. 7, World Sci., 1989, 138-177.

$[\mathrm{KW}] \quad$ V.G. Kac and M. Wakimoto, "Integrable highest weight modules over affine superalgebras and number theory", in: Progress in Math. 123, Birkhauser, Boston, 1994, pp. $415-456$.

[L] S.-R. Lu, "Some results on modular invariant representations", in Adv. Ser. in Math.Phys. 7, World Sci., Singapore, 1989, pp. 235-253.

[M] D. Mumford, Tata lectures on theta I, Progress in Math. 28, Birkhauser, 1983.

[PS] I. Penkov and V. Serganova, "Representations of classical Lie superalgebras of type I", Indag. Math. N.S. 3(4) (1992), 419-456.

[P] A. Polishchuk, "M.P.Appell's function and vector bundles of rank 2 on elliptic curves", preprint math. AG/9810084

[R] U. Ray, "A character formula for generalized Kac-Moody superalgebras", preprint.

[S] V. Serganova, Automorphisms of complex simple Lie superalgebras and affine KacMoody algebras, thesis, Leningrad State University, 1988.

[Z] Y. Zhu, "Modular invariance of characters of vertex operator algebras", J. AMS 9 (1996), 237-302. 